\title{
Wind Tunnel Investigation of Ground Wind Loads for Ares Launch Vehicle
}

\author{
Donald F. Keller ${ }^{*}$ and Thomas G. Ivanco ${ }^{\dagger}$ \\ Aeroelasticity Branch, NASA Langley Research Center, Hampton, Virginia, 23681
}

\begin{abstract}
A three year program was conducted at the NASA Langley Research Center (LaRC) Aeroelasticicty Branch (AB) and Transonic Dynamics Tunnel (TDT) with the primary objective to acquire scaled steady and dynamic ground-wind loads (GWL) wind-tunnel data for rollout, on-pad stay, and on-pad launch configurations for the Ares I-X Flight Test Vehicle (FTV). The experimental effort was conducted to obtain an understanding of the coupling of aerodynamic and structural characteristics that can result in large sustained wind-induced oscillations (WIO) on such a tall and slender launch vehicle and to generate a unique database for development and evaluation of analytical methods for predicting steady and dynamic GWL, especially those caused by vortex shedding, and resulting in significant WIO. This paper summarizes the wind-tunnel test program that employed two dynamically-aeroelastically scaled GWL models based on the Ares I-X Flight Test Vehicle. The first model tested, the GWL Checkout Model (CM), was a relatively simple model with a secondary objective of restoration and development of processes and methods for design, fabrication, testing, and data analysis of a representative ground wind loads model. In addition, parametric variations in surface roughness, Reynolds number, and protuberances (on/off) were investigated to determine effects on GWL characteristics. The second windtunnel model, the Ares I-X GWL Model, was significantly more complex and representative of the Ares I-X FTV and included the addition of simplified rigid geometrically-scaled models of the Kennedy Space Center (KSC) Mobile Launch Platform (MLP) and Launch Complex 39B primary structures. Steady and dynamic base bending moment as well as model response and steady and unsteady pressure data was acquired during the testing of both models. During wind-tunnel testing of each model, flow conditions (speed and azimuth) where significant WIO occurred, were identified and thoroughly investigated. Scaled data from the Ares I-X GWL model test was used in the determination of worst-case loads for the analysis of Ares I-X FTV design wind conditions. Finally, this paper includes a brief discussion of the limited full-scale GWL data acquired during the rollout and on-pad stay of the Ares I-X FTV that was launched from KSC on October 28, 2009.
\end{abstract}

Notice to readers:

The predicted performance and certain other features and characteristics of the Ares I and Ares I-X launch vehicles are defined by the U.S. Government to be Sensitive But Unclassified (SBU). Therefore, details have been removed from plots and figures.

\section{Nomenclature}

$1 \mathrm{Y}=1^{\text {st }}$ bending mode $-\mathrm{Y}$-direction

$1 \mathrm{Z}=1^{\text {st }}$ bending mode $-\mathrm{Z}$-direction

$2 \mathrm{Y}=2^{\text {nd }}$ bending mode - Y-direction

$2 \mathrm{Z}=2^{\text {nd }}$ bending mode - Z-direction

a $\quad=$ speed of sound, fps

$\mathrm{A}=$ area

$\mathrm{AB}=$ Aeroelasticity Branch (NASA LaRC)

\footnotetext{
* Senior Aerospace Research Engineer, Aeroelasticity Branch, Mail Stop 340.

${ }^{\dagger}$ Senior Aerospace Research Engineer, Aeroelasticity Branch, Mail Stop 340, AIAA Member.
} 


\begin{tabular}{|c|c|}
\hline AGL & $=$ Above Ground Level \\
\hline B-L & $=$ boundary layer \\
\hline $\mathrm{C} / \mathrm{Cc}$ & $=$ damping ratio, percent critical $(\% \mathrm{Cr})$ \\
\hline CAMI & $=$ Coated Abrasive Manufacturers Institute \\
\hline CAREA & $=$ planform area in $^{2}$ \\
\hline CFD & $=$ computational fluid dynamics \\
\hline CLV & $=$ Crew Launch Vehicle \\
\hline $\mathrm{C}_{\mathrm{M}}$ & $=$ base bending moment coefficient \\
\hline $\mathrm{C}_{\mathrm{MD}}$ & $=$ base bending moment coefficient due to drag \\
\hline $\mathrm{C}_{\mathrm{ML}}$ & $=$ base bending moment coefficient due to lift \\
\hline $\mathrm{CM} / \mathrm{LAS}$ & $=$ Command Module $/$ Launch Abort System \\
\hline $\mathrm{D}$ & $=$ diameter \\
\hline $\mathrm{DAC}$ & $=$ Design Analysis Cycle \\
\hline DAS & $=$ data acquisition system \\
\hline DBM & $=$ base bending moment due to drag, in- $-\mathrm{lb}_{\mathrm{f}}$ \\
\hline ESP & $=$ electronically scanned pressure \\
\hline FEM & $=$ finite element model \\
\hline fps & $=$ feet per second \\
\hline $\mathrm{f}$ & $=$ frequency, $\mathrm{Hz}$ \\
\hline $\mathrm{f}_{\mathrm{s}}$ & $=$ vortex shedding frequency, $\mathrm{Hz}$ \\
\hline FSS & $=$ Fixed Service Structure \\
\hline FTV & $=$ Flight Test Vehicle \\
\hline$F_{Y}$ & $=$ force in the $\mathrm{Y}$-direction, $\mathrm{lb}_{\mathrm{f}}$ \\
\hline $\mathrm{F}_{\mathrm{Z}}$ & $=$ force in Z-direction, $\mathrm{lb}_{\mathrm{f}}$ \\
\hline GWL & $=$ ground wind loads \\
\hline GWL CM & I $=$ Ground Wind Loads Checkout Model \\
\hline GWL TT & $=$ Ground Wind Loads Turntable \\
\hline $\mathrm{H}$ & $=$ wind-tunnel total pressure, $\mathrm{psf}$ \\
\hline $\mathrm{KSC}$ & $=$ Kennedy Space Center \\
\hline kts & $=$ knots (nautical miles per hour) \\
\hline $\mathrm{L}$ & $=$ length \\
\hline LaRC & $=$ Langley Research Center \\
\hline LAS & $=$ Launch Abort System \\
\hline $1 b_{f}$ & $=$ pounds force \\
\hline LBM & $=$ base bending moment due to lift, in- $-\mathrm{lb}_{\mathrm{f}}$ \\
\hline LC-39B & $=$ Launch Complex 39B (KSC) \\
\hline M & $=$ Mach number \\
\hline MLP & $=$ Mobile Launch Platform \\
\hline MS & $=$ model station, in \\
\hline $\mathrm{M}_{\mathrm{Y}}$ & $=$ base bending moment in $Y$-direction, in- $\mathrm{lb}_{\mathrm{f}}$ \\
\hline $\mathrm{M}_{\mathrm{Z}}$ & $=$ base bending moment in Z-direction, in- $\mathrm{lb}_{\mathrm{f}}$ \\
\hline OML & $=$ outer mold line \\
\hline PSD & $=$ power spectral density \\
\hline q & $=$ dynamic pressure, $\mathrm{psf}$ \\
\hline $\operatorname{Re}$ & $=$ Reynolds number \\
\hline RPM & $=$ revolutions per minute \\
\hline RSS & $=$ root sum squared (referring to data analysis) \\
\hline RSS & $=$ Rotating Service Structure \\
\hline SRB & $=$ solid rocket booster \\
\hline St & $=$ Strouhal number \\
\hline TDT & $=$ Transonic Dynamics Tunnel \\
\hline \multicolumn{2}{|c|}{ THETATT $=$ turntable angle, degrees } \\
\hline USAA & $=$ upper stage access arm \\
\hline $\mathrm{V}$ & $=$ test section flow velocity, fps \\
\hline $\mathrm{V}_{\mathrm{W}}$ & $=$ wind velocity, full-scale, knots (uniform flow) \\
\hline VAB & $=$ Vehicle Assembly Building \\
\hline
\end{tabular}

American Institute of Aeronautics and Astronautics 


$\begin{array}{ll}\text { VS } & =\text { vehicle station (full-scale), in } \\ \text { VSS } & =\text { Vehicle Stabilization System } \\ \text { WIO } & =\text { wind induced oscillation } \\ \mu & =\text { fluid viscosity, } \mathrm{lb}-\mathrm{sec} / \mathrm{ft}^{2} \\ \rho & =\text { fluid density, } \mathrm{s} / / \mathrm{ft}^{3} \\ \sigma & =\text { standard deviation "sigma" for dynamic signal, equivalent to RMS for zero mean }\end{array}$

\section{Introduction}

The Ares I-X Flight Test Vehicle (FTV) was rolled out and launched in October 2009 from Kennedy Space Center (KSC) Launch Complex (LC) 39B where the launch vehicle was exposed to surface winds during rollout and on the launch pad prior to launch. Structural design requirements developed for the vehicle stated that the Ares I-X FTV be designed to prevent instabilities and excessive wind-induced oscillations (WIO) and dynamic response due to vortex shedding produced by ground winds and gusts during the rollout, pre-launch, and launch phases of operation. This vehicle, by test and/or analysis, had to be shown to meet all program design requirements for strength, stiffness, and integrity for steady and dynamic loads that were the result of surface winds at KSC. In addition, there could not be a significant negative impact of winds on ground operations and pre-launch activities.

The ground wind loads (GWL) test and analysis efforts for the Ares I-X FTV involved wind-tunnel testing, and empirical and computational based analyses, of all relevant vehicle configurations. The primary objective of the two wind-tunnel tests was to provide data for direct evaluation of launch vehicle design, rollout and pad stay operations, and immediately prior to vehicle launch for wind conditions specified in the applicable design requirement documents. The data was also to be used for correlation to, and evaluation of, analytical methods being employed, or under consideration for use, in the ground wind loads assessment effort for the Ares I-X FTV and later for the planned Ares I Crew Launch Vehicle (CLV). GWL wind-tunnel models were required to be dynamically similar and incorporate all critical structural and aerodynamic elements of the vehicles and adjacent structures that could impact vehicle response to ground winds during rollout and pad stay although model and wind-tunnel test scope and requirements depended on the technical objectives of each test. The wind-tunnel tests were required to simulate, to the extent practical, all potential ground wind conditions that could be encountered at KSC.

The tests summarized in this paper are of the GWL Checkout Model (CM) and Ares I-X GWL Model in the NASA Langley Research Center (LaRC) Transonic Dynamics Tunnel (TDT). These tests were a critical part of the work performed to verify that the Ares I-X FTV would satisfy all applicable design requirements for ground wind loads during rollout to, and pad stay at, KSC LC-39B. The March/April 2007 test of the GWL CM served as a preliminary step in this verification effort with the objective of acquiring GWL data on a relatively simple Ares GWL model along with the secondary objective to reestablish, verify, and, where practical, improve GWL model design and testing capabilities within NASA. The Ares I-X GWL model was a more complex and realistic simulation of the Ares I-X FTV during rollout and pad stay. As such, the test configurations for rollout consisted of the Ares I-X FTV model mounted to a balance, that simulated the asymmetric stiffness that results from the launch vehicle hold down post geometry on the Mobile Launch Platform (MLP), and a simplified MLP to simulate aerodynamic effects of the Ares I-X FTV. The pad stay configurations included the addition of the Fixed Service (FSS) and Rotating Service (RSS) Structures of KSC's LC-39B represented by rigid model hardware to capture aerodynamic effects of these components on steady and dynamic GWL's. An internal tunable damper was employed for a limited portion of the rollout configuration testing to evaluate the impact of increased damping on the WIO and dynamic response characteristics of the launch vehicle model and to indirectly support design and analysis of the planned and employed, pad stay only, Vehicle Stabilization System (VSS).

These tests served as the primary source of experimental GWL data in the development and verification of analytical and computational methods for use in the Ares program and direct quantitative and qualitative GWL information for the Ares I-X FTV and, to a lesser extent, the planned Ares I CLV. A secondary objective of the Ares GWL wind-tunnel program was to continue to restore and improve the ground wind loads model design and testing capabilities within NASA that had not been employed in earnest since the late 1980's.

\section{A. Ground Wind Loads}

While being moved to and while at the launch pad, launch vehicles are typically exposed to steady winds and gusts and to the turbulent wake of nearby structures such as a launch tower, masts, and buildings. The response of the vehicle to the winds and turbulence can produce significant steady and oscillatory (dynamic) loads that must be accounted for in the design of the vehicle and support structures and in the development of ground and launch operation plans. In addition, the natural wind environment can be unpredictable and severe at times so a vehicle 
must be designed to withstand the wide range of wind conditions (speeds and azimuths) that could occur over the period of days, weeks, or even months that it will be on the launch pad.

Ground wind loads can be viewed as being comprised of three components, each of which can be further defined in terms of lift and drag. Viewing the vehicle from above, drag is defined to act on the vehicle cross section parallel to the wind velocity and lift is defined to act perpendicular $\left(90^{\circ}\right)$ to the wind velocity. A graphic of the wind components acting on a vehicle and a nearby structure from Ref. 1 is shown in Fig. 1.

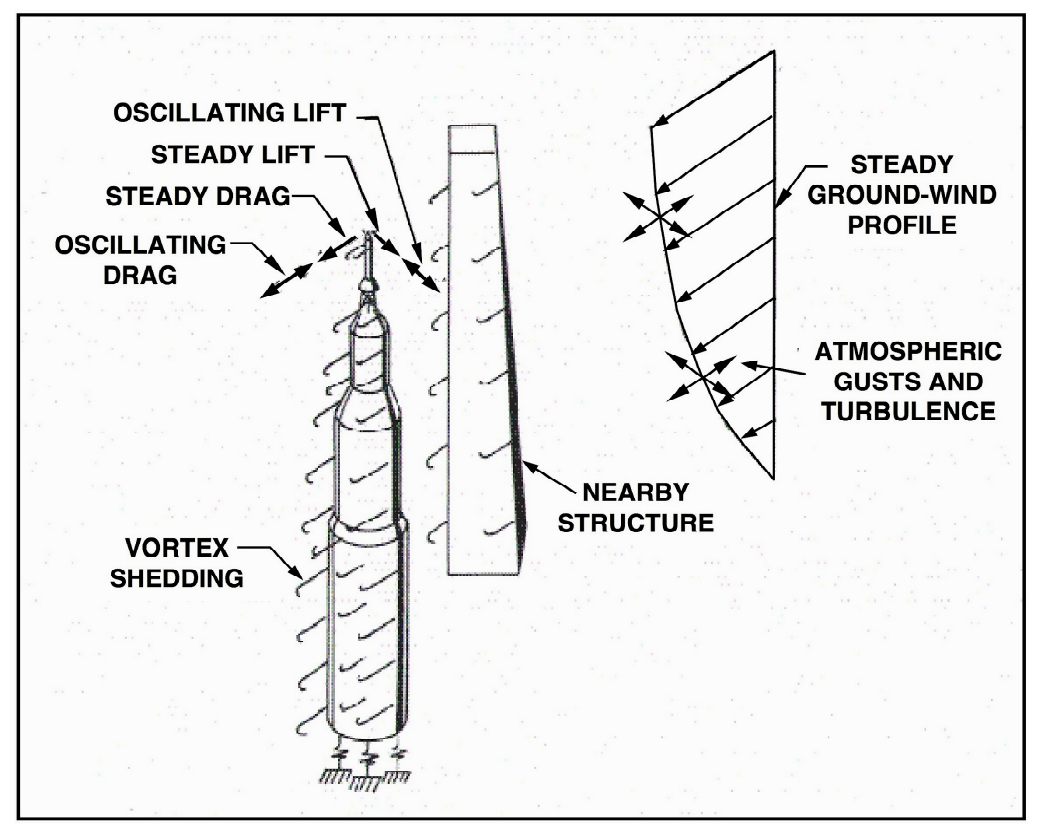

Figure 1. Ground wind loads components.

The first component is steady state wind loading that is defined in terms of steady state lift and drag coefficients that can be used to define loads on the launch vehicle. Steady state lift and drag coefficients, and their resulting loads, are typically a function of a launch vehicle's outer mold line (OML) and the kinematic properties of the air flow within a wind velocity range. The steady ground-wind profile illustrated in Fig. 1 is generally replaced with a steady uniform flow in wind-tunnel testing. Typically, steady drag loads are significantly larger than the steady lift loads and are a key consideration in launch vehicle and attachment structure design.

The second wind loading component is a result of wind gusts and turbulence. Gust loading is often applied to the launch vehicle in the form of a power spectral density (PSD) distribution in the drag direction. Wind-tunnel tests generally do not attempt to define gust loading on a launch vehicle since tunnel flow is generally uniform and does not contain representative gust and turbulence components. PSD information for use in analysis is usually generated based on wind gust data acquired at the launch site over an extended period of time.

The third component, and the focus of the Ares ground wind load testing in the TDT, is the dynamic loading component that results when a von Karman vortex street, created by periodic unsteady separation of flow along a portion or the entire length of the vehicle, forms in the wake of a launch vehicle. This vortex shedding and the resulting periodic lateral forces, perpendicular to the wind flow, can result in significant dynamic lift forces and moments on the vehicle. Vortex shedding loads are a function of wind velocity, characteristic diameter, of which there can be several depending on vehicle stage geometry, wind profile and flow characteristics, and the frequency (typically, a natural mode of vibration) and amplitude at which a vehicle responds. Large oscillatory loads can result if the frequency of the vortex shedding is at or near one of the natural frequencies of the vehicle (typically first bending mode but second bending mode can also be a concern) and there is insufficient damping in the vehicle to dissipate the energy being imparted to the vehicle. This phenomenon is sometimes referred to as "lock-on" or "lock in" and can produce oscillatory loads, great enough to damage the vehicle or levels of motion, WIO, that affect guidance system alignment, ground operations, and clearance between the vehicle and adjacent launch tower structures. Lock-on is also sometimes defined as a special case of WIO where the structural motion of the launch 
vehicle causes an alteration of the natural vortex shedding frequency such that the vortex shedding frequency matches the structural frequency of the mode aligned orthogonal to the flow direction over a narrow range of wind velocities. In effect the vehicle motion drives the frequency at which vortex shedding occurs. If a sufficient level of damping is present in the vehicle, then the response, while still at the fundamental frequency, is at much lower levels and more random in amplitude. For this case, the response is similar to that of a lightly damped mechanical system to a random input as described in Ref. 2 and illustrated in Fig. 2.

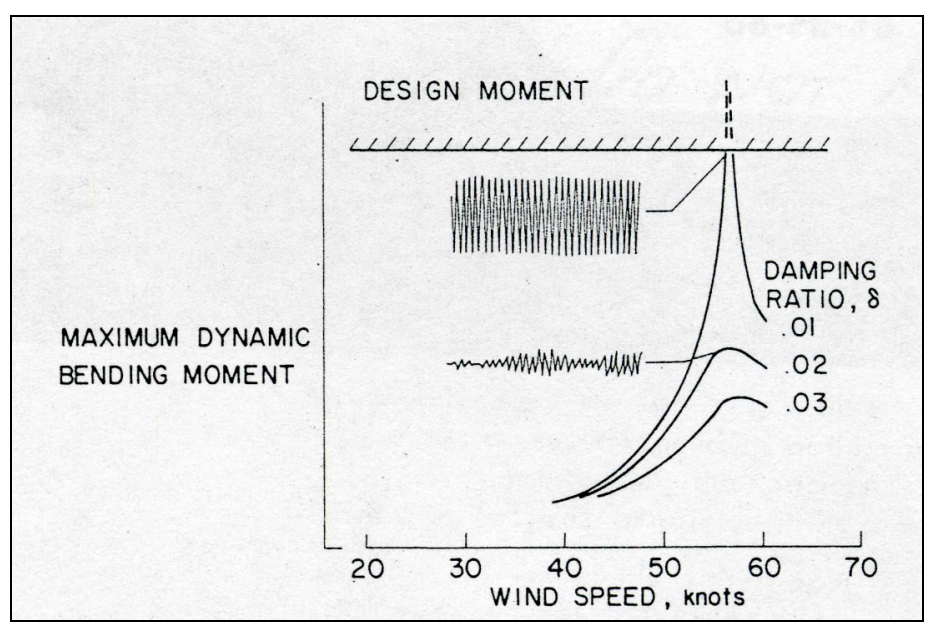

Figure 2. Data from Saturn $V$ wind-tunnel tests illustrating the effect of structural damping on dynamic bending moment.

As a result of ground winds, the launch vehicle structure (or wind-tunnel model) experiences a dynamic response and structural loads that can be categorized as steady (mean) and dynamic. The resulting structural loads can be directly measured with a wind-tunnel model and be resolved in the drag and lateral (lift) directions. A conceptual plot illustrating the components of these loads viewed from above is shown in Fig. 3. The response envelope depicted in Fig. 3 circumscribes the shaded area that represents a notional time-history trace of base bending moment. In Fig. 3, base bending moment due to drag is depicted horizontally, and base bending moment due to lift is depicted vertically.

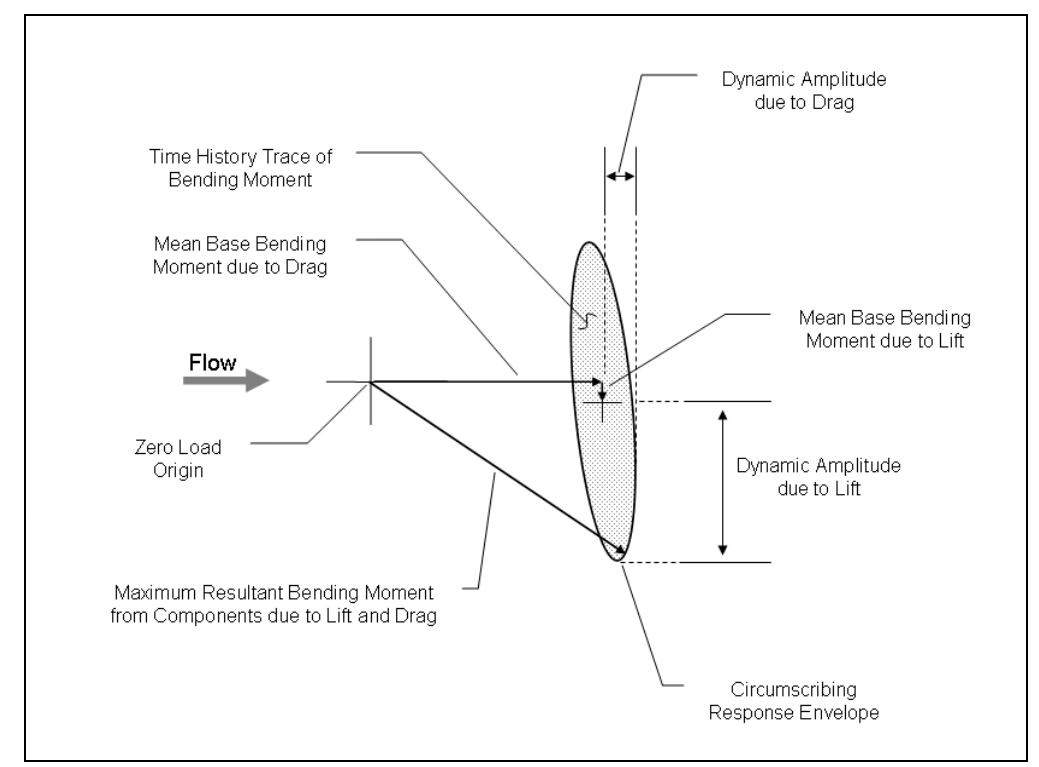

Figure 3. Components of measured base bending moment. 
Past ground wind load tests and studies of vortex shedding have indicated that vortex shedding loads can be very sensitive to surface roughness and protuberances, especially those protuberances that run along the length of a vehicle such as a fuel feed line or cabling conduit. Launch towers, buildings, and other nearby structures can also significantly affect vortex-shedding characteristics as a result of blockage, increased turbulence, periodic vortex shedding off a nearby structure, and venturi effects as nearby structures may constrict flow and result in locally higher wind velocities and wind azimuth variations. Some of these effects can result in increased vehicle dynamic loads. In general, vortex shedding loads are the most difficult to predict through analysis and the accepted method for prediction and determination of quantitative dynamic ground wind loads, especially for new launch vehicles, is by wind-tunnel testing ${ }^{3}$. It may be difficult, and require significant engineering judgment and data analysis, however, to determine and quantify the portion of a launch vehicle model dynamic response caused by wind-tunnel turbulence, nearby model structures, and vortex shedding during wind-tunnel testing. Wind-tunnel testing can be employed to test prospective fixes if ground wind loads are determined to be a potential concern. The most common fix is the use of a damper, attached to the vehicle from the launch tower or other nearby structure, that dissipates the energy transferred to the vehicle by the vortex shedding.

In general, through analysis and wind-tunnel testing, the three loading components described above are resolved independently and then combined to form the overall loading of a vehicle. The overall loadings are then used to evaluate the launch vehicle's structural integrity and ground wind launch availability and develop restrictions and placards for vehicle rollout, ground operations, and launch. It is important that, to the extent practical/possible, ground wind analyses and testing closely simulate these loading components.

\section{B. Reynolds Number and Strouhal Number}

The flow separation and the resulting shed vortices are highly dependent on Reynolds number and Strouhal number, non-dimensional parameters that are important to match in the design of any ground wind loads model and test plan in order to ensure that the results are applicable and scalable to the actual launch vehicle. Strouhal number is often used to describe the occurrence of vortex shedding. Details for scaling of ground wind loads models and tests are presented later in this paper. Strouhal number for a cylinder is defined in Eq. (1) as:

Strouhal number $=S_{t}=f_{s} D / V$

where $f_{s}$ is the vortex shedding frequency in $\mathrm{Hz}, D$ is the cylinder diameter, and $V$ is inflow velocity, or test section velocity for the purpose of this paper. Reynolds number, fur this paper, is defined in Eq. (2) as:

Reynolds number $=\operatorname{Re}=\rho \mathrm{VD} / \mu$

where $\rho$ and $\mu$ are the density and viscosity of the air or test medium.

Experimental data and theory suggest that Strouhal number for a long cylinder is in the vicinity of 0.20 over a wide range of Reynolds number, although this can vary significantly depending on surface roughness and protuberances ${ }^{4}$. At Reynolds numbers between approximately 300 and $3 \times 10^{5}$, the boundary layer is laminar over the front portion of the cylinder; over the rear portion of the cylinder the boundary layer separates and breaks up into a turbulent wake. The separation points move forward on the cylinder as the Reynolds number is increased. As Reynolds number increases beyond $3 \times 10^{5}$, depending on surface roughness and free stream turbulence, the flow separation points move backward, the drag drops sharply, and the vortex shedding is disorganized. At higher Reynolds number, values above $2.5 \times 10^{6}$ to $3.5 \times 10^{6}$, strong vortex streets are reestablished and significant periodic lifting forces imparted on the cylinder.

The relationship between Reynolds number and Strouhal number for long circular cylinders, based on data from several sources ${ }^{4}$ is presented in Fig. 4. It should be noted that this graphic is based on data for cylinders where 2-D flow predominates. No verified data is known to exist for determining Strouhal number for short cylinders over a range of flow conditions and therefore Fig. 4 should be used only as a guide for ground wind loads analysis and testing. Experimental evidence does exist that suggests three-dimensional flow, especially that found near the top of a launch vehicle, significantly affects local Strouhal number and results in vortex shedding frequencies in those regions to be significantly different from other regions on a vehicle that experience flow that is more twodimensional in nature. 


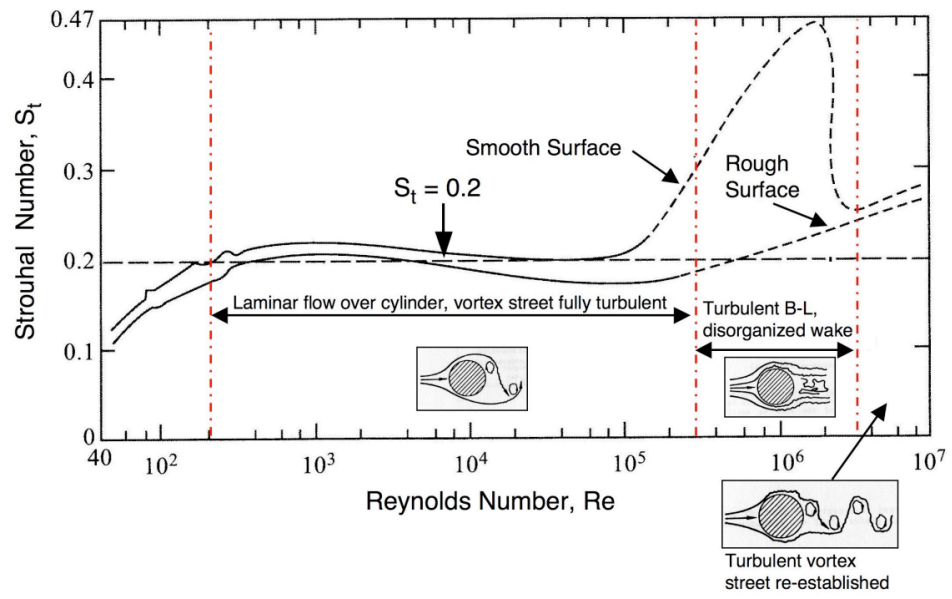

Figure 4. Relationship between Strouhal number and Reynolds number for long circular cylinders.

Ground winds profiles are typically specified as a function of height and, as a result, local wind velocities along the length of a vehicle vary significantly between the base and top of a launch vehicle, thereby varying both the Strouhal and Reynolds number along the length of a launch vehicle. The natural environment wind conditions specified for the Ares I-X FTV for rollout (and rollback, if needed), pad stay (including ground operations) and launch are presented in Table 1. These winds, identified for rollout and pad-stay, were specified for a reference height of $60-$ feet $^{5}$. The specified wind exposure wind profile as a function of wind speed at the reference height is shown graphically in Fig. 5.

Table 1. Ares I-X FTV Natural Environment Wind Conditions.

\begin{tabular}{|c|c|c|c|c|}
\hline Condition & $\begin{array}{c}\text { Exposure } \\
\text { Period }\end{array}$ & $\begin{array}{c}\text { Risk of Exceeding } \\
\text { Wind Speed at Ref. } \\
\text { Height }\end{array}$ & $\begin{array}{c}\text { Peak Winds at 60' } \\
\text { Ref. Height. } \\
\text { knots/mph }\end{array}$ & $\begin{array}{c}\text { Mean Winds at 60' } \\
\text { Ref. Height. } \\
\text { knots/mph }\end{array}$ \\
\hline Rollout/Rollback (design) & 1 Day & $1 \%$ & $47.0 / 54.1$ & $31.1 / 35.8$ \\
\hline Rollout/Rollback (target) & 1 Day & - & $30.5 / 35.1$ & $20.0 / 23.0$ \\
\hline Pad Stay (design) & 30 Days & $1 \%$ & $65.0 / 74.8$ & $43.2 / 49.7$ \\
\hline Ground Operations & - & - & $30.5 / 35.1$ & $20.0 / 23.0$ \\
\hline Launch & 1 Hour & $5 \%$ & $20.0 / 23.0$ & $12.7 / 14.6$ \\
\hline
\end{tabular}




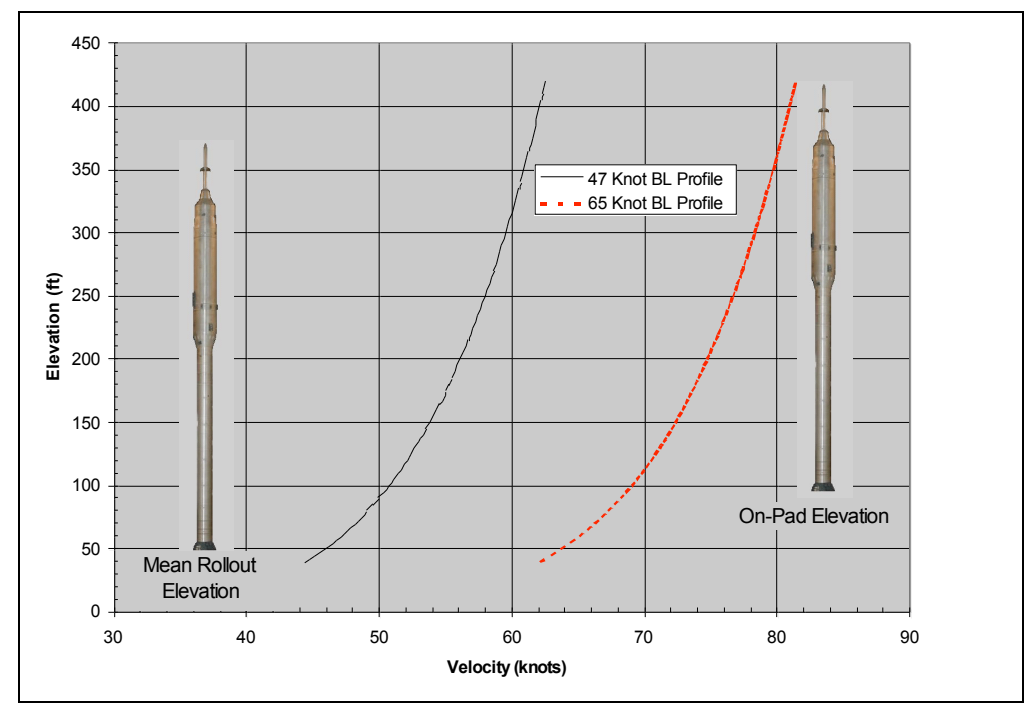

Figure 5. KSC boundary layer profile for reference height (60 feet above ground level (AGL)) velocities of 47 and 65 knots.

\section{Ares Launch Vehicle and GWL}

The Ares I-X FTV and the planned Ares I CLV had geometric and dynamic characteristics that required analysis and testing to investigate potential ground wind loads issues. Compared to other launch vehicles, the Ares I-X FTV and Ares I CLV have an unusually high ratio of overall vehicle length to diameter, or fineness ratio (see Fig. 6) with an upper stage that was approximately $50 \%$ larger in diameter than the first stage. The first stage would be a Space Shuttle solid rocket booster (SRB) modified with an additional fifth segment for increased duration of thrust during ascent for the Ares I CLV. The additional fifth segment for the Ares I-X FTV was an inert OML/mass simulator. The first stage and upper stage for the Ares I-X FTV was each of near constant diameter. This geometry could result in large regions of vortex shedding to occur on the vehicle at specific wind conditions. In addition, aerodynamic loads imparted to the upper stage could result in unacceptably high base bending moments due to their relatively large distance from the base of the vehicle. This loading condition would be especially critical if strong vortex shedding occurred on the upper stage at a frequency at or near a vehicle natural mode of vibration in combination with significant drag loads in high wind conditions. Numerous protuberances on the Ares I-X FTV, especially the systems tunnel that ran nearly the entire length of the vehicle, could have also significantly affected steady and dynamic loading of the vehicle depending on wind speed and azimuth.

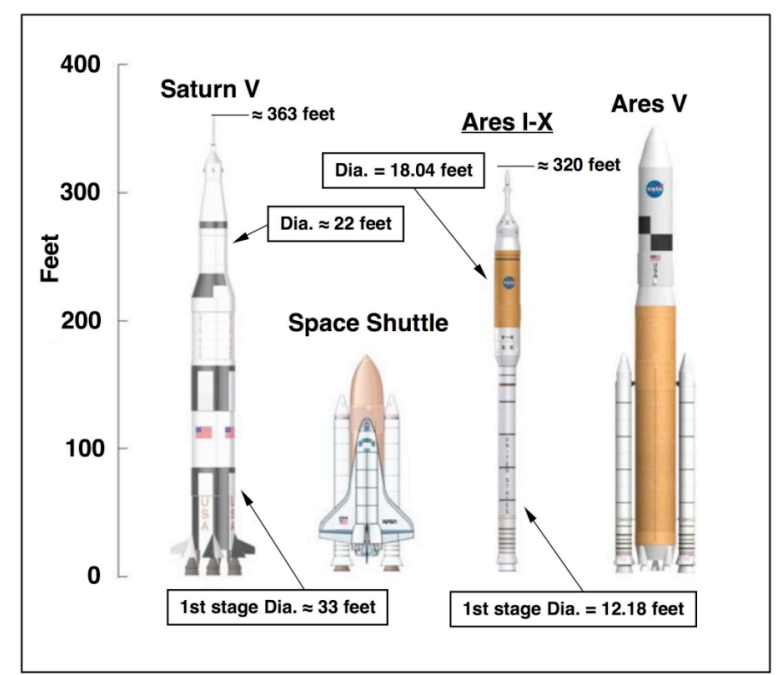

Figure 6. Launch vehicle comparison. 
Due to the hold-down post configuration at the base of the Ares I-X FTV, and the resultant asymmetric stiffness at the vehicle base, the Ares I-X FTV had greater deflection in the Y-axis direction (East-West direction at KSC LC39B) than in the Z-axis direction (North-South) for a given load. This hold-down post geometry also resulted in the vehicle being predicted to have two orthogonal first bending and second bending modes of vibration (four total modes) with the mode shapes oriented in the $\mathrm{Y}$ and $\mathrm{Z}$ axes. The hold-down post geometry at the base of the Ares IX FTV is illustrated in Fig. 7. There was also concern that the Ares launch vehicles could have low inherent values of structural damping for first and second bending modes of vibration. Measurement of the actual values for structural damping for the Ares I-X FTV was attempted prior to rollout and results from the Ares I-X FTV modal test are presented later in this paper. Based on historical data for launch vehicles, a value of $\approx 0.5 \% \mathrm{C} / \mathrm{C}_{\mathrm{c}}$ was assumed as a worst-case value for the purpose of wind-tunnel test planning and associated analyses.

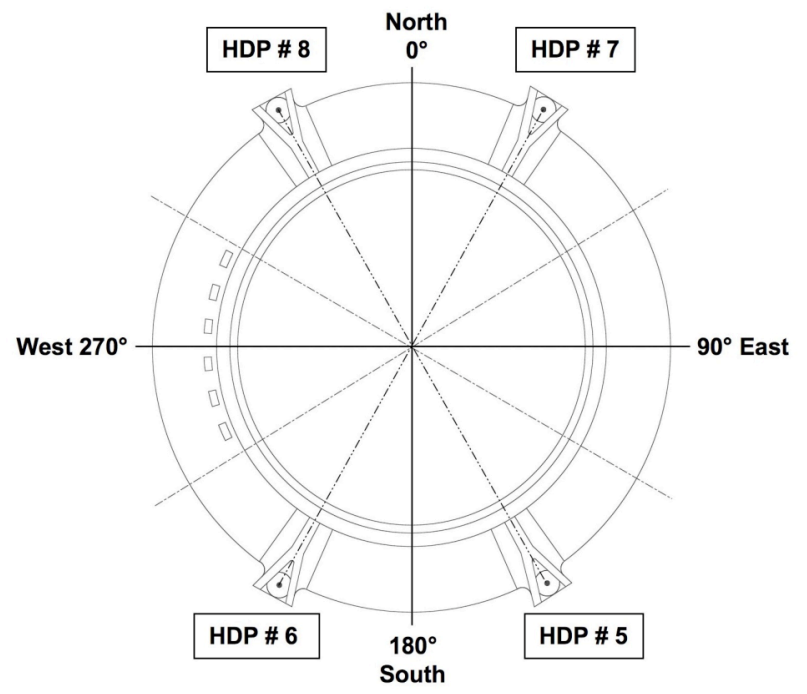

Figure 7. SRB aft skirt hold-down posts (HDP's) with compass directions for the Ares I-X FTV on the MLP at KSC Launch Pad 39B

As with the Space Shuttle and Saturn V vehicles, the Ares I-X FTV needed several hours to be transported on the MLP/Crawler from the Vehicle Assembly Building (VAB) to the launch pad and, during this period, was especially vulnerable to high velocity winds. The Ares I-X FTV was unrestrained during rollout except for the four hold-down post attachments at the base of the vehicle. Due to the launch vehicle's predicted low natural frequency for its first two modes of vibration (first-bending mode frequencies for Ares I-X FTV were predicted to be 0.17 and $0.21 \mathrm{~Hz}$ for Y-axis and Z-axis, respectively) the wind velocities at which vortex shedding might "lock in" with this mode could be rather low (approximately 6 to 12 knots at reference height of 60 feet above ground level (AGL)). The low steady drag and lift loads along with relatively weak vortex shedding loads were viewed as not likely to result in significant base bending moment loads although vehicle motion could have been significant if wind velocity remained at a relatively constant critical value and inherent vehicle damping was low.

Vortex shedding typically excites the first bending modes of launch vehicles. The dynamic responses of these modes are usually the focus of WIO analyses and wind-tunnel tests. As a result of significant first bending mode deflections and load distributions, ground wind loads typically drive the strength requirements for the lower portion of the launch vehicle. The Ares I-X FTV, however, had small section diameters and low predicted natural frequencies. Therefore, the wind speed ranges where vortex shedding frequencies could align with the natural frequencies of the second bending modes were within the design ground wind speed envelope. Additionally, the load distribution associated with a second bending mode deflection is considerably different than that of a first bending mode deflection. Figure 8 contains notional, yet representative, bending moment distributions for first and second bending mode responses. These load distributions are normalized to a base bending moment magnitude of one and are derived from load analysis of the Ares I-X FTV finite element model (FEM). For the same magnitude of base bending moment, one can easily see that many areas of the vehicle could be subjected to considerably higher loads for a second mode response by comparison to a first mode response. Therefore, ground wind loads were also a concern on how they could affect strength requirements for other areas of the Ares I-X in addition to the vehicle base region. 


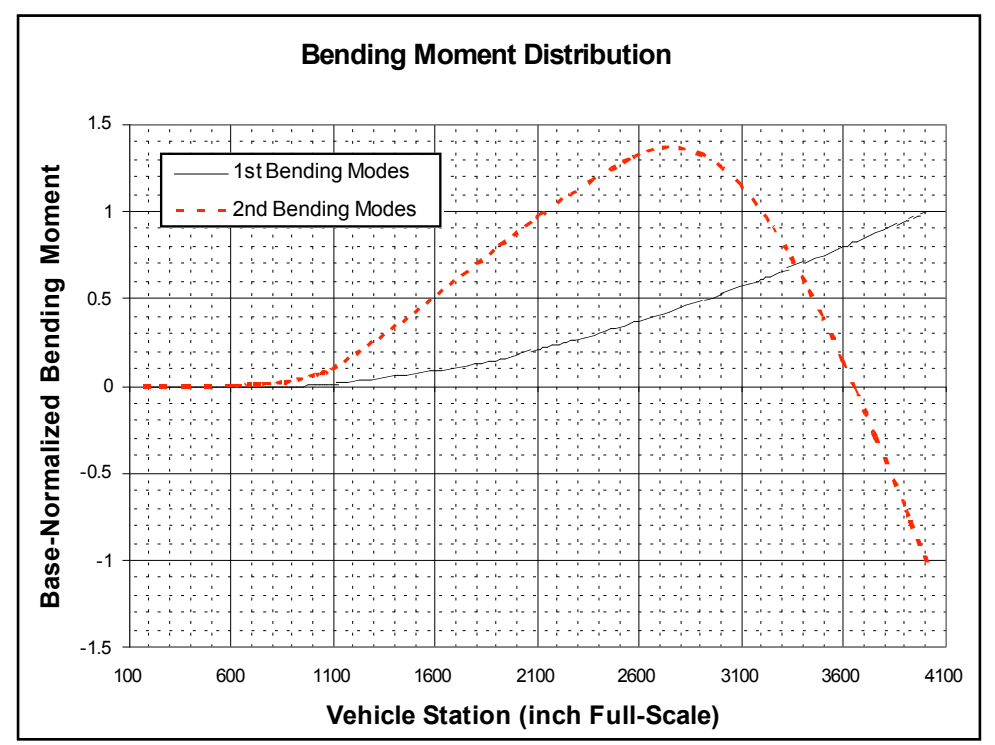

Figure 8. Notional bending moment distribution associated with first and second bending modes response.

Documented experimental data showing such an occurrence of a significant second bending mode response due to vortex shedding, WIO, for a launch vehicle had not been found. Investigating this unique case of second bending mode response was a unique focus of the Ares I-X ground wind loads wind-tunnel test. Due to the vehicle's predicted second bending mode frequencies of 1.01 and $1.16 \mathrm{~Hz}$, in the $\mathrm{Y}$ and $\mathrm{Z}$ axis, respectively, there was concern that strong vortex shedding could occur at high wind velocities that would "lock in" with this second mode of vibration. These dynamic loads, combined with the resultant higher steady loads, were a concern with regard to vehicle structural integrity at the vehicle base and in the region of the fifth segment of the first stage. Large dynamic motion in proximity to launch tower structures was also a concern although the on-pad VSS was planned and ultimately implemented for the vehicle. The VSS, a system of springs and dampers, was attached to the vehicle in the interstage region during pad stay after rollout to LC-39B had been completed. The VSS was detached from the vehicle prior to launch. A listing of Strouhal numbers and velocities for vortex shedding based on predicted Ares IX FTV natural frequencies and first and second-stage diameters is presented in Table 2. The velocities presented in the table assume uniform flow (uniform ground wind profile) along the length of the specified vehicle stage. Determination of a launch vehicle Strouhal number for critical configurations across a range of possible wind conditions was important for the Ares ground wind loads analysis and testing program. The current practice was to determine those values of Strouhal number through wind-tunnel testing and, if possible, with modern computational methods although this is currently impractical due to very large computational requirements.

Table 2. Velocities for 1st and upper stages of Ares I-X FTV where vortex shedding could "lock-in" with critical vehicle modes of vibration. Assumes uniform flow (uniform ground wind profile).

\begin{tabular}{|c|c|c|c|c|}
\multicolumn{5}{c}{ First Stage Upper Stage } \\
\hline Strouhal & $f_{I Y,} f_{I Z}=0.17,0.21 \mathrm{~Hz}$ & $f_{2 Y,} f_{2 Z}=1.01,1.16 \mathrm{~Hz}$ & $f_{I Y,} f_{I Z}=0.17,0.21 \mathrm{~Hz}$ & $f_{2 Y,} f_{2 Z}=1.01,1.16 \mathrm{~Hz}$ \\
\hline 0.16 & $V_{s}=7.7,9.5 \mathrm{knots}$ & $V_{s}=45.6,52.3 \mathrm{knots}$ & $V_{s}=11.4,14.0 \mathrm{knots}$ & $V_{s}=67.5,77.5 \mathrm{knots}$ \\
\hline 0.20 & $6.1,7.6$ & $36.4,41.9$ & $9.1,11.2$ & $54.0,62.0$ \\
\hline 0.24 & $5.1,6.3$ & $30.4,34.9$ & $7.6,9.4$ & $45.0,51.7$ \\
\hline 0.28 & $4.4,5.4$ & $26.0,29.9$ & $6.5,8.0$ & $38.6,44.3$ \\
\hline 0.32 & $3.8,4.7$ & $22.8,26.2$ & $5.7,7.0$ & $33.7,38.7$ \\
\hline
\end{tabular}

\section{Technical Approach}

The two Ares wind-tunnel models discussed in this paper were conceived and developed in response to the requirement for experimental ground wind loads data to support Ares launch vehicle development. The GWL 
Checkout Model was conceived and developed in the summer of 2006 and tested in the spring of 2007. This model served as an important basis for the development, design, and testing of the more complex and representative Ares IX GWL model that occurred over the period of mid-2007 through fall 2008. Each model and associated windtunnel test, while supporting the same overall objective, had similar and different aspects to the technical approach taken to support the successful acquisition and analysis of scaled experimental GWL data to support the Ares I launch vehicle program.

\section{A. Scope and Objectives}

\section{GWL Checkout Model}

The GWL Checkout Model (CM) was designed and fabricated at the NASA Langley Research Center (LaRC) under the direction of the NASA LaRC Aeroelasticity Branch (AB). The AB staff developed a concept for a simple low-cost 4-percent length-scaled ground wind loads model that allowed for a full checkout of the TDT in the conduct of a GWL test. In addition, it provided a low risk opportunity for $\mathrm{AB}$ researchers to go through the process of designing, fabricating, testing, and reporting on a GWL test prior to the actual Ares I-X test scheduled for Spring 2008 while acquiring test data for early identification and evaluation of potential Ares GWL issues.. The GWL CM was based on the then available Ares I design data and dynamic finite element model. The GWL CM was required to be low cost, simple to fabricate (no launch tower was considered), and ready for testing in the spring of 2007. The primary test configurations were the launch vehicle model with and without protuberances. Additional parameters varied during the test were Reynolds number and surface roughness.

\section{Ares I-X GWL Model}

The $\mathrm{AB}$ researcher staff involved in the earlier GWL CM project began preliminary design of the 4-percent scaled Ares I-X GWL model, and planned tunnel test in the TDT, in the summer of 2007 using lessons learned from the earlier GWL CM test and the information then available for the Ares I-X FTV, Mobile launch Platform (MLP), and primary KSC LC-39B structures.

The Ares I-X FTV was considered a somewhat unique launch vehicle in that there was potential for significant WIO to occur for any of the first four primary modes of vibration; first and second bending in the $\mathrm{Y}$ and $\mathrm{Z}$ axes. There was also the concern that vehicle response to ground winds could vary significantly between rollout and pad stay due to the proximity of the primary KSC LC-39B launch pad features, the Fixed Service (FSS) and Rotating Service (RSS) Structures. Wind requirements for rollout and pad stay were significantly different from each other with higher wind requirements needed to be met for the period the Ares I-X FTV would be at the launch pad. As a result, two primary configurations were tested, the Ares I-X FTV during rollout on the MLP and the launch vehicle on the MLP at the KSC LC-39B, to determine upper-allowable and/or critical wind speeds and azimuth angles at which significant levels of WIO could occur for ground wind conditions, specified as possible, during vehicle rollout and on-pad stay and launch configurations. Inherent vehicle damping was unknown until ground testing of the Ares I-X FTV, so structural damping was determined to be an additional critical parameter to vary during the wind-tunnel test. No attempt was made to simulate the planned Vehicle Stabilization System (undefined at the time of model definition and design) and variation in vehicle model damping was accomplished using an internal tunable mass-spring viscous damper.

\section{B. Caveats and Limitations}

Ground wind loads wind-tunnel testing cannot fully simulate an actual launch vehicle in natural wind conditions. Therefore, caveats and limitations associated with the inability to simulate certain components of the full-scale vehicle and wind environment and limitations associated with the wind-tunnel facility and models were accepted to meet the objectives of the Ares GWL experimental program. The caveats and limitations listed below, some model specific, were the more significant considered before test results were used to make decisions regarding vehicle structural analysis and, ground and launch operations.

1. Wind-tunnel flow was uniform and did not accurately simulate the steady ground wind boundary-layer profile at KSC as illustrated in Fig. 5. All wind-tunnel results were analyzed for steady uniform flow without a boundary layer. In order to relate the wind-tunnel data to an appropriate reference height velocity, it would be required to determine the velocity mapping that will present the most representative loads. The recommended method of determining an acceptable velocity map is to find a representative uniform velocity that produces the same mean bending moment due to drag as that created with a given reference height velocity and boundary layer profile. ${ }^{6}$

2. Wind-tunnel gust and turbulence levels did not simulate the natural wind turbulence at KSC; therefore, the associated loads and vehicle response were not measured. Wind gusts will most likely increase the 
total bending moment load due to drag beyond the static load for an equivalent peak velocity as a result of structural oscillations. Furthermore, Ref. 7 mentions that free stream turbulence can increase the strength of vortex shedding in addition to broadening the frequency bandwidth.

3. The effect of tunnel blockage and test section walls (including boundary layer) on test data, particularly for the Ares I-X GWL model when the FSS and RSS were installed, were unknown. The cross sectional area of the rollout configuration for the Ares I-X and all configurations tested for the GWL CM were considered acceptable. The additional blockage caused by the addition of the FSS and RSS to simulate the on-pad stay configurations for Ares I-X may have caused some unquantified effects on the data.

4. Reynolds number as a function of reduced frequency was limited to approximately $30 \%$ of full-scale values for the Ares I-X GWL model by test section geometry (model size), test medium, and the requirement to limit test section velocity to the quasi-incompressible flow regime (Mach number $\leq$ 0.30 although some data was acquired out to $\mathrm{M}=0.35$ ). The $\mathrm{GWL} C \mathrm{CM}$ was tested at approximately correct Reynolds number as a result of model design scale point and to a $30 \%$ increase in launch vehicle model. Simulation of higher effective Reynolds number for the Ares I-X GWL model was accomplished using fine grit applied to the entire surface of the launch vehicle model to accelerate supercritical flow transition. Still, unsteady behavior that occurs in the transcritical Reynolds number range, prior to transition to supercritical, tends to be more difficult to simulate with a subscale test since the flow field is chaotic as opposed to structured into single-frequency shedding.

5. Compromises were made regarding the level of detail of the MLP, FSS, and RSS structures for the Ares I-X GWL Model. No MLP was present for the GWL CM. Simplification of the model hardware was inevitable and the test team utilized judgment to simulate the significant components.

6. The GWL CM was mounted to a rigid base plate that did not simulate the structural restraint conditions (asymmetric stiffness) of the Mobile Launch Platform (MLP).

7. Compared to the anticipated inherent structural damping of the Ares I-X FTV, the wind-tunnel models were believed to have relatively low inherent structural damping. Limited damping variations were accomplished for the Ares I-X GWL Model with an internal variable damper instead of one that would more closely simulate the mechanics and operation of the planned vehicle stabilization system (VSS). In addition, some unwanted documented variation in damping occurred as a result of turntable mechanical characteristics, especially for the GWL CM, and these may have impacted the test results for both models.

\section{Model Orientations}

\section{GWL Checkout Model}

The coordinate systems and sign conventions for the GWL CM, GWL TT, and data are shown in Fig. 9. A photo of the model at a GWL TT angle (flow azimuth) of $0^{\circ}$ is shown in Fig. 10. Note that the model center of rotation were offset slightly upstream and toward the test section West wall. The model therefore translated a small distance as the GWL TT was rotated. This location was selected to use existing threaded holes in the GWL TT surface and the desire to mount the model in the center of the GWL TT to allow for passage of instrumentation umbilicals through the center hole of the GWL TT. 


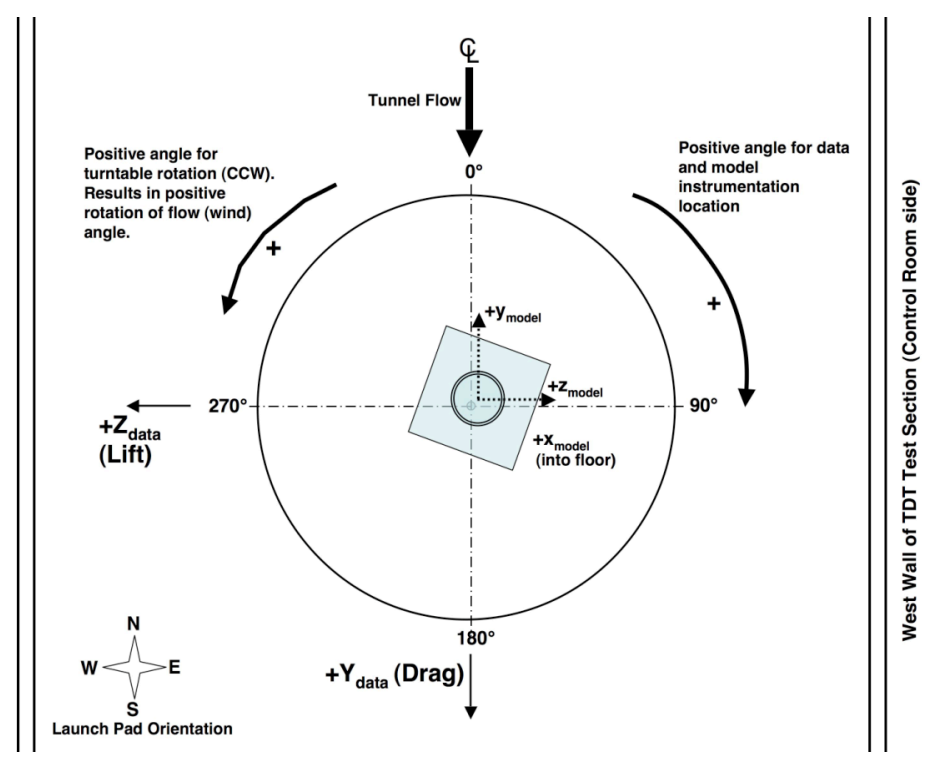

Figure 9. Coordinate system and layout for GWL CM mounted in the TDT Test Section. Flow azimuth $=0^{\circ}$.

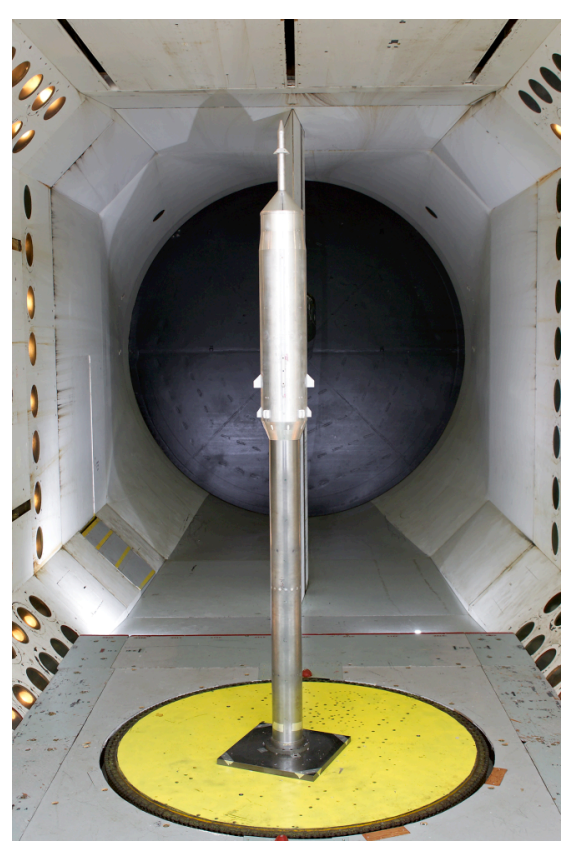

Figure 10. GWL CM mounted on the GWL TT in the TDT. Flow azimuth $=0^{\circ}$. Looking downstream.

\section{Ares I-X GWL Model}

The Ares I-X FTV model structural (model-axis) body coordinate system was selected to be the same as used for the full-scale vehicle and is shown in Fig. 11. This figure depicts the definition of the X, Y, Z model-axis system that is identical in orientation to the flight test vehicle (FTV) structural body coordinate system. Also shown in the inset in Fig. 11 is the definition of model azimuth angle. Identical to the FTV azimuth angle, the model azimuth angle is zero at the positive Z-direction, $90^{\circ}$ at the systems tunnel, and $270^{\circ}$ at the positive Y-direction. The orientation of the launch vehicle model coordinate system with relation to the KSC LC-39B model hardware was also selected to be identical to that of the full-scale vehicle as illustrated in Fig. 12. 


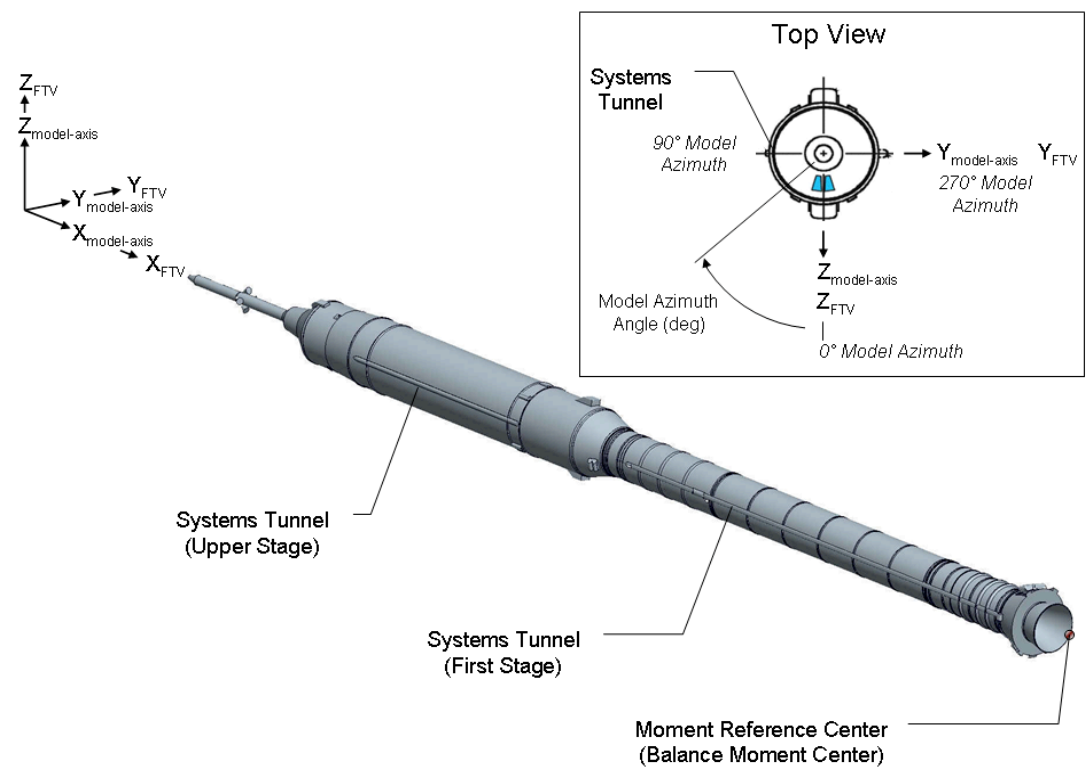

Figure 11. Definition of Ares I-X launch vehicle model-axis coordinate system.

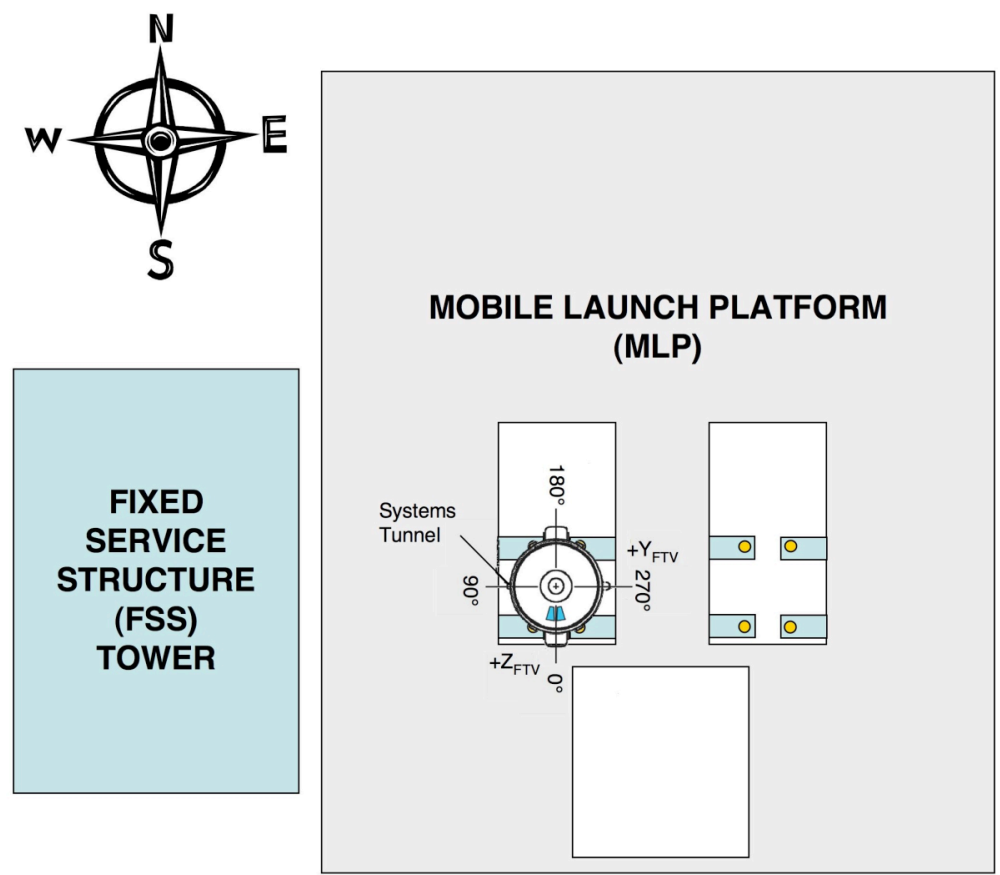

Figure 12. Ares I-X FTV orientation on KSC Launch Complex 39B.

\section{Dynamic Aeroelastic Scaling}

Model similitude to a full-scale vehicle requires wind-tunnel flow conditions and model structural characteristics to satisfy various gas-flow and structural relationships with the associated full-scale wind conditions and vehicle structure, respectively. The accurate simulation of steady and unsteady aerodynamic forces and resulting model responses was identified as very desirable since it would result in wind-tunnel data that could be used to better verify analysis and computational methods and be scaled to the full-scale Ares I-X FTV and perhaps in some approximate manner to the planned Ares I CLV. 
Important parameters for aeroelastic scaling of ground wind loads models include mass ratio, Reynolds number, reduced frequency, Strouhal number, Mach number, and Scruton number as defined in Eq. (3) through Eq. (8).

$$
\begin{aligned}
\text { mass ratio } & =\frac{\text { mass vehicle }_{\text {mass displaced fluid }}}{\text { Reynolds number }(\mathrm{Re})}=\frac{\rho \mathrm{VL}}{\mu} \\
\text { reduced frequency }\left(k_{H z}\right) & =\frac{f_{\text {structural mode }} D}{V} \\
\text { Strouhal number }(\mathrm{St}) & =\frac{f_{\mathrm{s}} D}{V} \\
\text { Mach number }(\mathrm{M}) & =\frac{V}{a} \\
\text { Scruton number }(\mathrm{Sc}) & =\frac{4 \pi m \varsigma_{j}}{\rho D^{2}}
\end{aligned}
$$

where:

$$
\mathrm{m}=\text { mass per unit length of the vehicle and } \varsigma_{j} \text { is the damping ratio of mode } j
$$

To establish aeroelastic similitude for ground wind loads testing, the values of the aeroelastic scaling parameters should be identical for the wind-tunnel model and full-scale vehicle. However, Mach number matching was assumed to be unnecessary as long as "incompressible" conditions were maintained during wind-tunnel testing and for the equivalent full-scale conditions. Mach numbers up to 0.30 were assumed to comply with the incompressible assumption. For this reason, most test data was acquired at or below this Mach number in the wind-tunnel tests, and no effort was made to simulate full-scale Mach number. Scruton number is sometimes referred to as a critical modeling parameter to characterize vortex shedding response. Scruton number is simply a combination of the mass ratio and damping ratio. Therefore, for the same value of structural damping, the wind-tunnel model Scruton number equals that for the full-scale flight vehicle. Finally, reduced frequency is defined here in a nonstandard manner using frequency in $\mathrm{Hz}$ in lieu of $\mathrm{rad} / \mathrm{s}$ and length as diameter in lieu of semi-chord. This nonstandard definition allows a direct comparison of reduced frequency with Strouhal number.

\section{Test Facility}

\section{A. Transonic Dynamics Tunnel}

The NASA Langley Research Center Transonic Dynamics Tunnel (TDT) has operated for fifty years supporting aeroelastic and non-aeroelastic research testing of fixed wing, rotorcraft, spacecraft, and miscellaneous unique aerospace vehicle configurations. The TDT is a continuous flow, single return, slotted-throat wind-tunnel capable of testing at total pressures from 0.01 to 1.0 atmospheres and over a Mach number range from zero to 1.20 . The test section of the TDT is $16.0 \mathrm{ft}$. square with cropped corners for a cross-sectional area of approximately 248 square feet. The TDT has specific features that make it ideally suited for studying aeroelastic and other unsteady flow phenomena. Testing can be conducted in the TDT using either air or a heavy gas, R-134a, as the test medium. Testing in R-134a provides advantages in aeroelastic model scaling due primarily to its higher density, approximately three times that of air, that also results in significantly higher possible Reynolds number flow conditions. The TDT is also equipped with a set of four quick-actuating bypass valves that can be activated to rapidly reduce test section velocity when necessary to prevent or minimize the risk of model and facility damage. An aerial photo and plan view of the TDT are presented in Fig. 13 and Fig. 14, respectively. The TDT operating envelope for R-134a as the test medium, with region of interest for GWL testing, is shown in Fig. 15. 


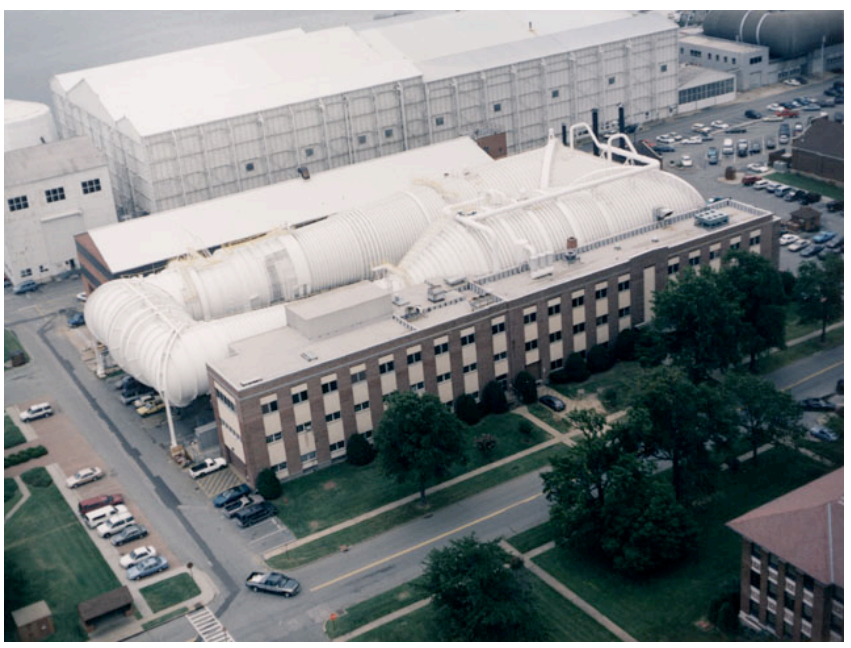

Figure 13. Aerial view of NASA LaRC TDT.

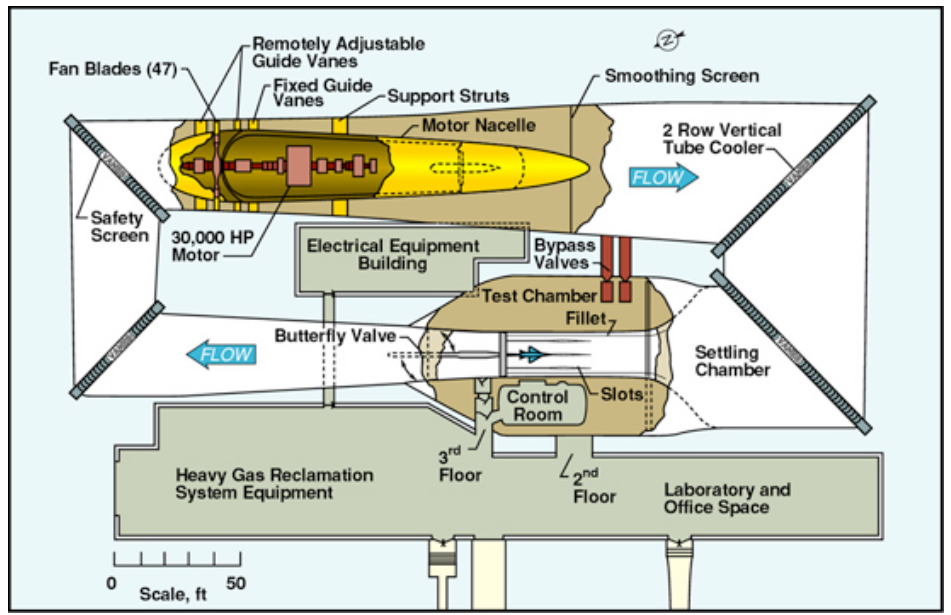

Figure 14. Plan view of NASA LaRC TDT. 


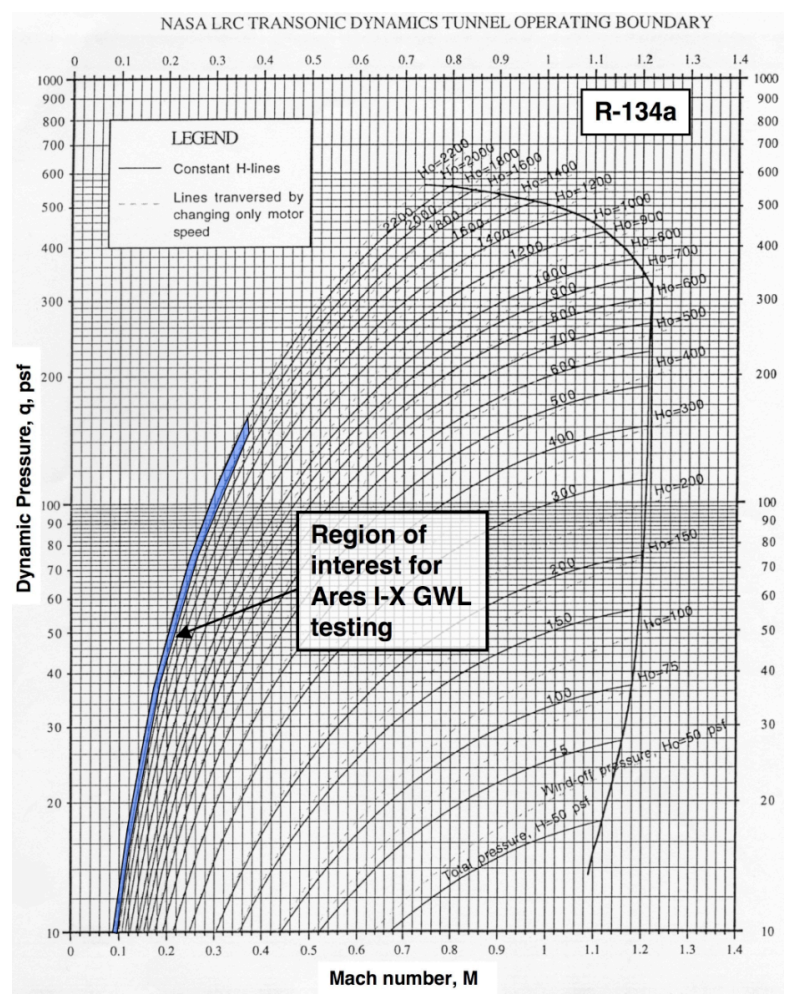

Figure 15. TDT operating boundary for R-134a as test medium.

\section{B. Ground Wind Loads Turntable}

The mount system for GWL testing in the TDT is the remotely operated Ground Wind Loads Turntable (GWL TT). The GWL TT was originally constructed and employed in the mid-1960s and used extensively for GWL testing in the 1960s and 1970s.

The GWL TT consists of an 8-foot diameter stationary steel base plate and a gas-floated (or suction-locked) 8foot diameter, 3 -inch thick rotating steel plate with a 12 -inch diameter center spindle. The upper plate can be gasfloated, using air or heavy gas, and then rotated at approximately one rpm by two electrical motor and gear box assemblies whose output sprockets engage a continuous roller chain attached to the outer edge of the rotating plate. Model instrumentation umbilicals are routed through the center of the spindle to the TDT East-wall platform patch panel. A photo of the GWL TT during installation is shown in Fig. 16.

Rotation of a model is accomplished through lifting the upper plate by introducing air or R-134a (when R-134a is the tunnel test medium) at eight pressure pads in the lower plate. A flexible sponge rubber (or similar material) seal is installed around the edge of the upper plate. The GWL TT seal is normally fully compressed when the table is unpowered or suction is applied to the pressure pads. This characteristic improves the contact pressure between the plates to provide a good repeatable boundary condition for model testing. The seal expands a small amount when the table is floated to increase the distance between the two plates during rotation. The table is floated on a gas film of $0.010-0.030$ inches depending on supply pressure, condition of the seal, model weight and location, and aerodynamic forces and moments acting on the model. The GWL TT can then be rotated to the desired angle and, when the desired rotation angle has been reached, the lifting pressure removed and a negative pressure (suction) applied to provide additional hold-down force and prevent the rotation of the GWL TT during acquisition of data.

Once the GWL TT is installed and readied for operation, a wooden floor/fairing is installed to cover the five-inch thickness of the GWL TT that projects above the floor of the TDT test section. A photo of the GWL TT, wood fairing, and Ares I-X GWL model rollout configuration installed in the TDT test section is shown in Fig. 17. 


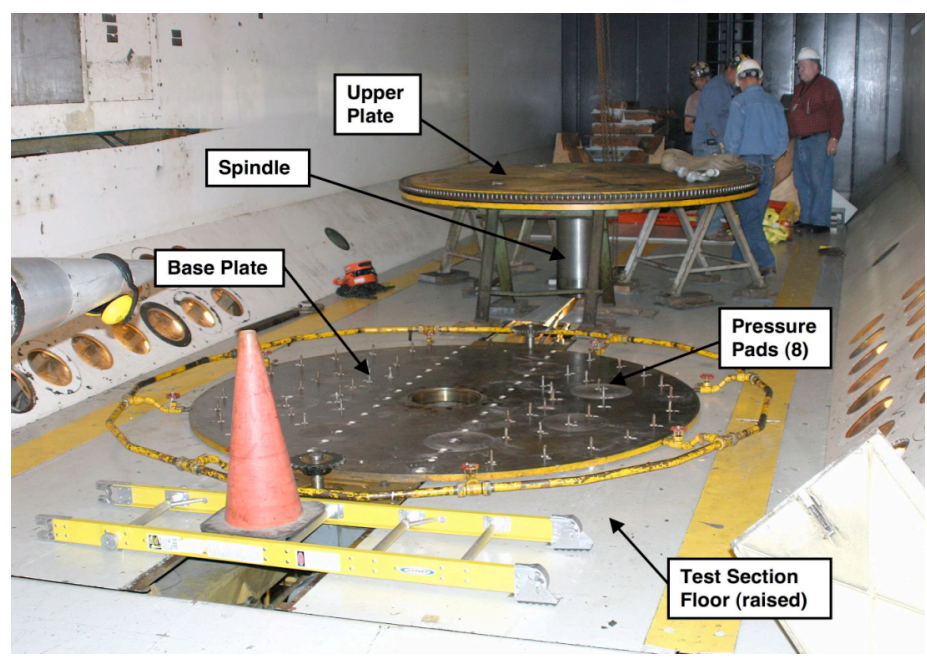

Figure 16. Ground Wind Loads Turntable (GWL TT) installation in TDT test section.

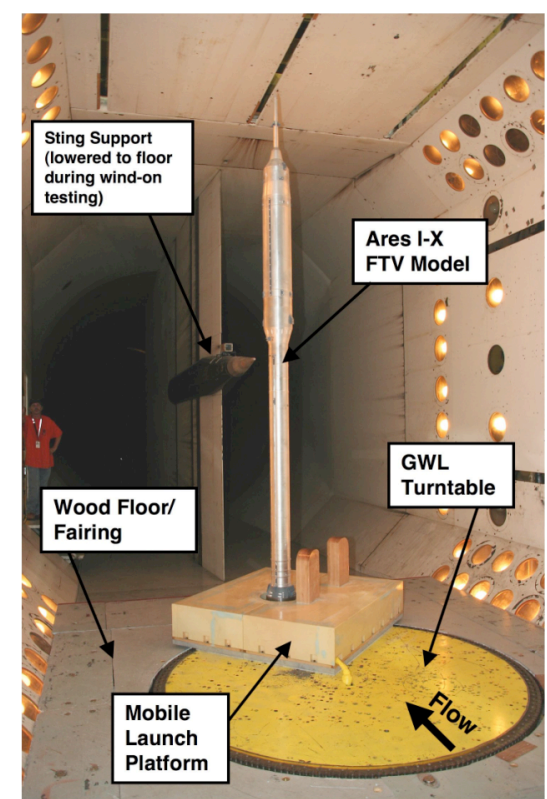

Figure 17. GWL TT with Ares I-X FTV and MLP model components installed in TDT test section.

\section{Model Descriptions}

\section{A. Ground Wind Loads Checkout Model}

\section{General Description}

The Ground Wind Loads Checkout model was a relatively simple, low cost, extensively instrumented, all metal launch vehicle model designed to provide initial parametric GWL data, with emphasis on conditions of high WIO, for the Ares I-X and Ares I CLV. The model, in many ways, served as a testbed for the Ares I-X GWL model. The actual Ares I-X FTV was not extensively defined at the time of GWL CM design. The GWL CM had a length scale factor of $4 \%$ that resulted in a model of approximately 13 feet in height. This was chosen to maximize the size of the model that would fit inside the TDT test section and not have the top of the model too close to the test section ceiling. The model was designed to have as few joints as practical and the joints had to be tight to minimize overall model inherent damping. Variation in model damping was to be accomplished using an internal viscous damper but a successful design was not completed for this model prior to testing. The outer mold line (OML) for the GWL CM was based on the Ares I Design Analysis Cycle 1 (DAC-1) that is illustrated by the solid model in Fig. 18. Primary protuberances for the Ares 1 DAC-1 were included on the model and were simplified and designed to be easily 
installed and removed during testing. The GWL CM was cantilevered at the base for simplicity and no launch tower was included due to schedule and cost constraints. The mass and stiffness characteristics for the full-scale Ares I, as defined in the then (June 2006) available NASTRAN finite-element model (FEM), was used as the basis for obtaining scaled values of mass and stiffness distributions for use in design of the wind-tunnel model. Sectional bending stiffness and mass information was extracted from the FEM as well as mode shapes and frequencies for the full-scale vehicle first and second bending modes of vibration. The model was designed to withstand dynamic pressures up to $110 \mathrm{psf}$ for testing in air or heavy gas, R-134a. The planform and layout of the model along with key model stations are shown in Fig. 19.

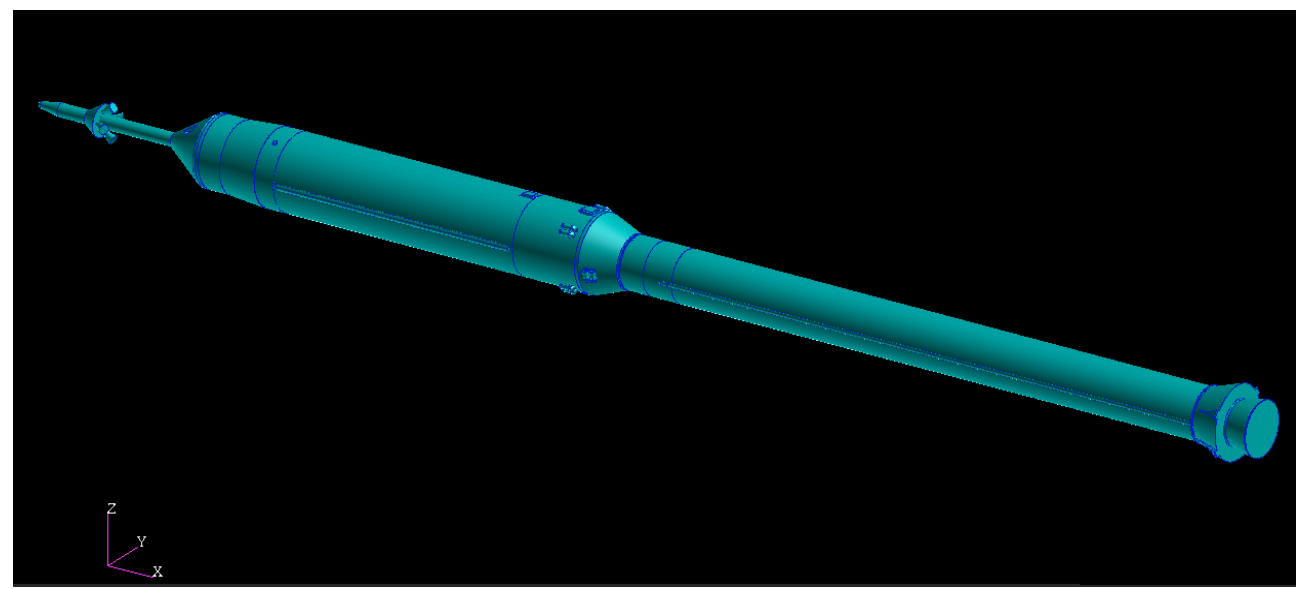

Figure 18. Ares I DAC-1 solid geometry.

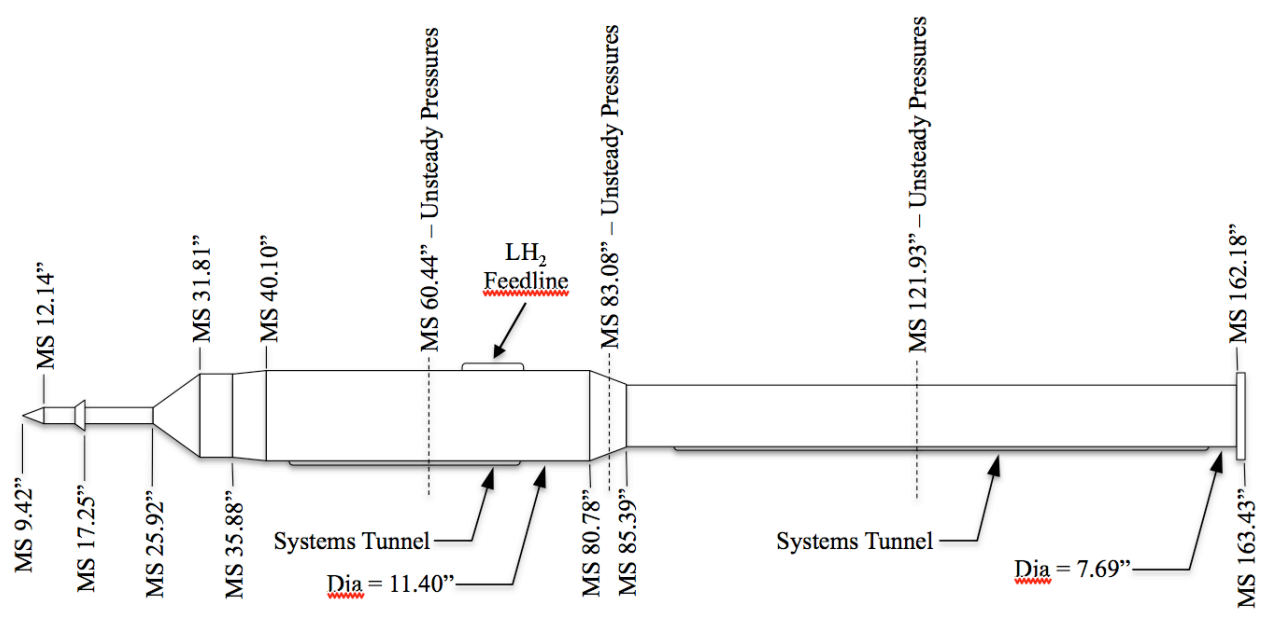

Figure 19. GWL CM planform and layout.

\section{Dynamic Aeroelastic Scaling and Design}

The accurate simulation of steady and unsteady aerodynamic forces and resulting model response was identified as very desirable since it would result in wind-tunnel data that could be used to better verify analysis and computational methods and, in at least some approximate manner, be scaled to the full-scale Ares I-X FTV and Ares I CLV.

Full-scale aerodynamic parameters in air were the values for standard sea level conditions. The assumed aerodynamic parameters for the TDT were those for R-134a at a pressure of $2000 \mathrm{psf}$, temperature of $560^{\circ} \mathrm{R}$ and purity of 95 -percent ( $5 \%$ air). Model geometric scale was chosen to maximize model size within the geometric and aerodynamic limitations of the TDT. A list of these parameters is presented in Table 3. 
Table 3. Parameters for GWL CM scaling.

(Air)

(R-134a)

\begin{tabular}{|l|c|c|c|}
\hline \multicolumn{1}{|c|}{ Parameters } & Full Scale & Model & Ratio (Model/Full) \\
\hline Density $\left(\mathrm{sl} / \mathrm{ft}^{3}\right)$ & $2.380 \times 10^{-3}$ & $7.200 \times 10^{-3}$ & 3.025 \\
\hline Viscosity $\left(\mathrm{lb}-\mathrm{sec} / \mathrm{ft}^{2}\right)$ & $3.737 \times 10^{-7}$ & $2.610 \times 10^{-7}$ & 0.698 \\
\hline Speed of Sound $(\mathrm{ft} / \mathrm{s})$ & $1.116 \times 10^{3}$ & $0.550 \times 10^{3}$ & 0.493 \\
\hline Vehicle Height $(\mathrm{ft})$ & 321 & 13 & 0.0405 \\
\hline
\end{tabular}

A standard dynamic-aeroelastic scaling was performed that resulted in a set of scale factors being generated that was used to scale the Ares I geometry and FEM derived information to use as a basis for GWL CM design.

Model response will be greatest when the vortex shedding frequency, as determined by Strouhal number, coincides with a vehicle mode of vibration. Fig. 20 presents a plot of reduced frequency versus Reynolds number for the full-scale Ares I vehicle where reduced frequency is defined by the vehicle upper stage diameter, $D$, predicted first mode frequency, $f$, and wind velocity, $V$. This definition is also the same as that for Strouhal number. A model that has the same relationship (curve) between reduced frequency and Reynolds number, although the corresponding wind-tunnel velocities will be different due to model geometric scale, first mode frequency, and test medium, should have dynamic response and vortex shedding characteristics that accurately simulate that of the fullscale vehicle over a wide range of full-scale ground wind velocities. This assumes that other factors such as structural damping, surface roughness, flow turbulence and wind profile are either properly simulated or do not have a significant effect on vortex shedding characteristics. If for example, the upper stage of the Ares I-X had a Strouhal number equal to 0.20 at a Reynolds number of approximately two million, the vehicle could be expected to have maximum vortex shedding response at a velocity of approximately $18 \mathrm{ft} / \mathrm{s}$ ( $\approx 10.6 \mathrm{knots})$. A Strouhal number of less or greater value would result in peak vortex shedding response occurring at higher or lower velocities, respectfully. The curve in Fig. 20, therefore, served as part of the basis for the scaling of the GWL CM .

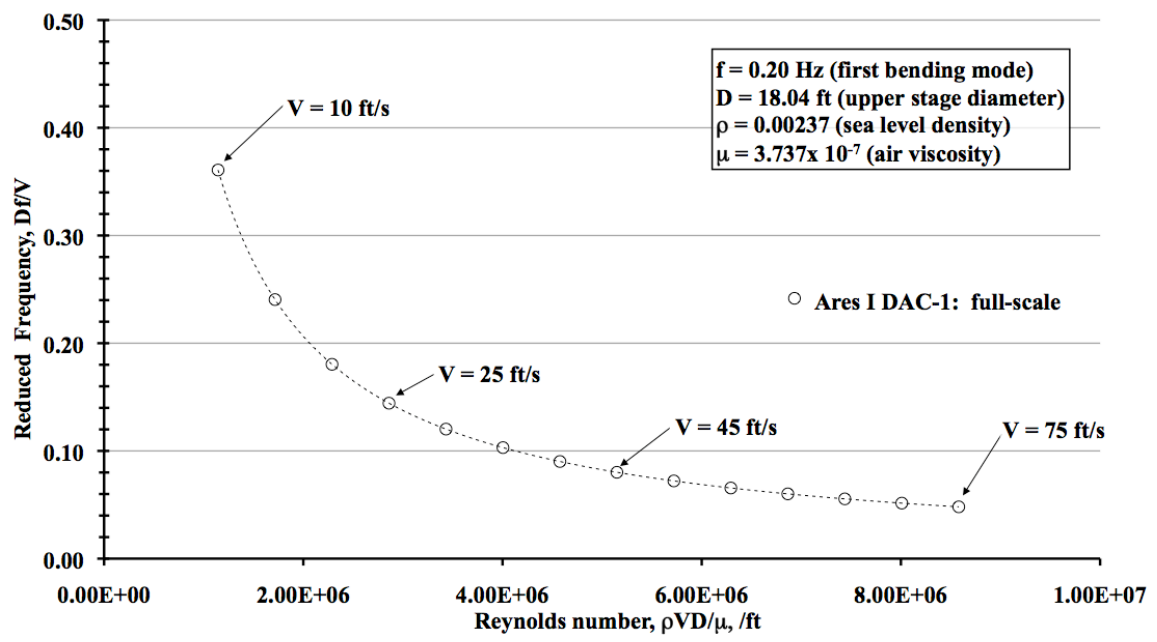

Figure 20. Reduced frequency vs Reynolds number for Ares I DAC-1.

The mass and stiffness characteristics for the full-scale Ares I as defined in the then available NASTRAN FEM was used as the basis for obtaining scaled values of mass and stiffness distributions for use in design of the windtunnel model. The mobile launch platform was removed from the FEM and the vehicle base modeled as cantilevered for the calculation of mode shapes and frequencies. The frequencies of the first two full-scale modes are presented in Table 4.

Table 4. Full-scale cantilevered modes for Ares DAC-1.

\begin{tabular}{|c|c|c|}
\hline Mode Number & Mode Name & Frequency, $\mathbf{H z}$ \\
\hline 1 & $1^{\text {st }}$ bending & $\approx 0.23$ \\
\hline 2 & $2^{\text {nd }}$ bending & $\approx 0.92$ \\
\hline
\end{tabular}

20

American Institute of Aeronautics and Astronautics 
The resulting initial scale factors are presented in Table 5. A wind velocity of $20 \mathrm{knots}(33.8 \mathrm{ft} / \mathrm{s})$ was chosen to demonstrate the resulting scaled velocity, Reynolds number, and Strouhal number for a specific velocity although any velocity could have been chosen as it would not have an effect on the key scale factors of mass, frequency, and bending stiffness.

Table 5. Initial GWL CM scale factors with full-scale and model values.

\begin{tabular}{|l|c|c|c|}
\hline \multicolumn{1}{|c|}{ Scale Factor } & Full-Scale Value & Scale ratio & Model-scale value \\
\hline Length & $321 \mathrm{ft}$ & 0.0405 & $13.0 \mathrm{ft}$ \\
\hline Diameter $\left(1^{\text {st }}\right.$ stage $)$ & 18.04 & 0.0405 & 0.731 \\
\hline Reynolds number & $3.9 \times 10^{6}$ & 1.0 & $3.9 \times 10^{6}$ \\
\hline Reduced freq. $\left(S_{t}\right)$ & 0.107 & 1.0 & 0.107 \\
\hline Velocity & $33.8 \mathrm{ft} / \mathrm{s}(20 \mathrm{kts})$ & 5.73 & $193.7 \mathrm{ft} / \mathrm{s}(\mathrm{M} \approx .35)$ \\
\hline Mass (weight) & $2.0 \times 10^{6} \mathrm{lbs}$ & $2.01 \times 10^{-4}$ & $402 \mathrm{lbs}$ \\
\hline Frequency & $f_{l}=0.23 \mathrm{~Hz}$ & 141.53 & $\begin{array}{c}f_{l}=32.6 \mathrm{~Hz} \\
f_{2}=0.92 \mathrm{~Hz}\end{array}$ \\
& \multicolumn{2}{|c}{} \\
\hline
\end{tabular}

Preliminary model design studies quickly showed that a model with a length of 13 feet and a weight of 400 pounds could not be designed to have a first mode frequency at or even near $32.6 \mathrm{~Hz}$. In addition, test section Mach number would have been required to be approximately 0.35 for a full-scale wind speed of 20 knots. It was therefore determined that the model would have to be "off-scale" in at least one or more parameters. It was decided that the model should be designed to the curve presented in Fig. 20 and that could be accomplished if the model diameter scaling was allowed to increase by 30 percent to 0.0527 while holding the length scale factor to the original value of 0.0405 . The increase in diameter would result in the model reduced frequency-Reynolds number curve rising above and to the right of the full-scale model curve. Model stiffness ( $1{ }^{\text {st }}$ mode frequency) would then have to be decreased to lower the model curve back down to the original curve. It was found that a 30 percent increase in diameter required the bending stiffness scale factor to be reduced to 32 percent of the original value. This increase in model diameter scale to make design and fabrication of the GWL CM practical, resulted in a set of model-scale values for frequency and stiffness that were achievable using common metallic materials in combination with a variable-wall thickness tube-section model concept.

The GWL CM first stage was a steel tube with the rest of the model structure fabricated using aluminum alloy. Wall thickness was varied to match running stiffness design targets. Two large ballast weights were installed in the first stage and two smaller ballast weights in stalled in the upper stage to match running mass requirements. The effect on model aerodynamic characteristics were unknown but were considered acceptable based on the GWL CM test objectives. The final scale factors and resulting model-scale values are presented in Table 6. Model mass (weight) increased to 462 pounds as a result of an error in extracting and summing mass information from the Ares I FEM as part of an effort to improve the accuracy of model mass and stiffness information. This error was discovered after model design was complete and fabrication had begun. Since primary project objectives would not be significantly affected, no attempt was made to correct the error and the final model was fabricated to the higher weight target. The full-scale and final model-scale reduced frequency-Reynolds number curves are presented in Fig. 21. The figure also presents model-scale velocities at selected points for comparison to full-scale ground-wind velocities for the Ares CLV. 
Table 6. Final GWL CM scale factors adjusted for 30-percent diameter scale increase.

\begin{tabular}{|l|c|c|c|}
\hline \multicolumn{1}{|c|}{ Scale Factor } & Full-Scale Value & Scale ratio & Model-scale value \\
\hline Length & $321 \mathrm{ft}$ & 0.0405 & $13.0 \mathrm{ft}$ \\
\hline Diameter $\left(1^{\text {st }}\right.$ stage $)$ & $18.04 \mathrm{ft}$ & 0.0527 & $0.950 \mathrm{ft}$ \\
\hline Reynolds number & $3.9 \times 10^{6}$ & 1.0 & $3.9 \times 10^{6}$ \\
\hline Reduced freq. $\left(S_{t}\right)$ & 0.107 & 1.0 & 0.107 \\
\hline Velocity & $33.8 \mathrm{ft} / \mathrm{s}(20 \mathrm{kts})$ & 4.44 & $150 \mathrm{ft} / \mathrm{s}(\mathrm{M} \approx 0.27)$ \\
\hline Mass (weight) & $2.3 \times 10^{6} \mathrm{lbs}$ & $2.01 \times 10^{-4}$ & $462 \mathrm{lbs}$ \\
\hline Frequency & $\begin{array}{l}f_{l}=0.23 \mathrm{~Hz} \\
f_{2}=0.92 \mathrm{~Hz}\end{array}$ & $\underline{71.7}$ & $\begin{array}{c}f_{l}=16.5 \mathrm{~Hz} \\
f_{2}=66.0 \mathrm{~Hz}\end{array}$ \\
\hline
\end{tabular}

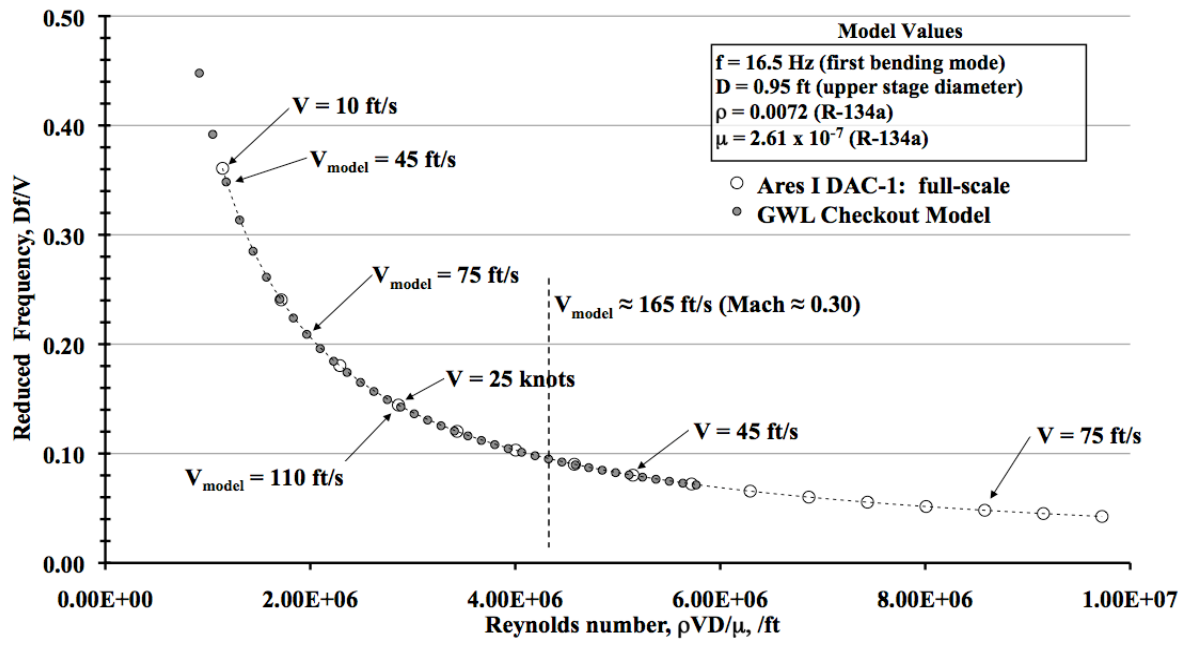

Figure 21. Reduced frequency vs Reynolds number for Ares DAC-1 and final GWL CM design.

\section{Instrumentation}

Experimental data was acquired using a variety of instrumentation consistent with to the objectives of the windtunnel test. The measurement of base bending moment loads, steady and dynamic, was accomplished using four Wheatstone-type strain gauge bridges located at the base of the model. The strain-gauge bridges were calibrated after the model was installed in the test section by applying a series of positive and negative loads 75.5 inches up (MS 85) from the base of the model in the axis of each bridge. Load checks were performed prior to wind-on testing to insure accuracy of $\pm 10 \%$ of applied moment. During testing, it was found that gauge drift was an issue and the tunnel flow was stopped regularly to zero out the base bending moment gauges to minimize effects of drift on the data.

Two vertical rows of fifteen piezoelectric-type accelerometers mounted on the $0^{\circ}$ and $90^{\circ}$ model azimuths and distributed evenly along the length of the model were employed to acquire model dynamic response data. The accelerometers were bonded to the inside of the model surface or mounting blocks using cyanoacrylate (super glue) adhesive. The accelerometers were calibrated prior to installation in the model.

Sixteen miniature, high-sensitivity piezoresistive unsteady pressure transducers were mounted in-situ at three model stations; $60.43,83.08$, and 121.93 . These pressure transducers were evenly spaced around the circumference of the model at each of the model stations for a total of 48 model unsteady pressure transducers with an integral orifice at each location. The unsteady pressure transducers were used to acquire steady and unsteady static-pressure data. The pressure transducers were calibrated after installation in the model using a reference pressure tube located at the rear of each transducer.

Twenty-five static-pressure orifices were located on the model for acquisition of steady pressures along the $90^{\circ}$ model azimuth. These orifices were nearly equally spaced (with exceptions) from the base to the top of the model. Flexible pressure tubing was attached to each of the orifices and routed to two 16-port Electronically Scanned 
Pressure (ESP) modules located underneath the TDT test section. The ESP modules were calibrated and the orifices leak checked several times during the test.

\section{Configurations}

The original baseline configuration, configuration $\# 1$, for the GWL CM test was the model without protuberances or surface grit as shown in Fig. 22. This configuration was tested with air as the test medium at near atmospheric pressure for the purpose of checkout and troubleshooting of instrumentation and model/facility systems, and to obtain data at relatively low Reynolds number flow conditions. Baseline model damping was determined to be near or greater than 1-percent critical and additional increases in damping during testing were not required and/or desired.

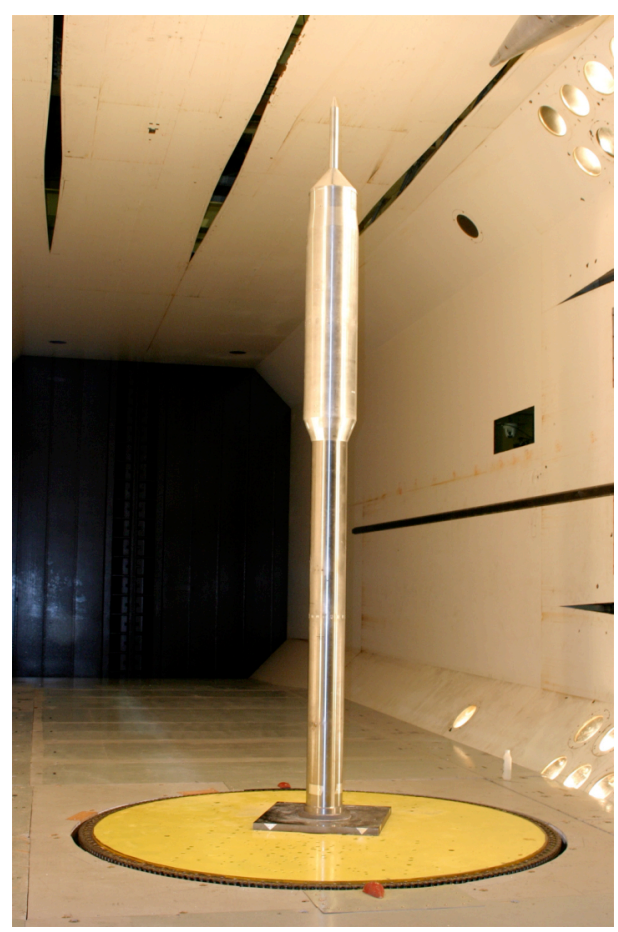

Figure 22. GWL CM Configuration \#1, baseline - looking upstream.

Protuberances were added to the baseline configuration and additional air data acquired to investigate the effects of protuberances in low Reynolds number flow conditions and for comparison to similar and higher Reynolds number data that was later acquired with R-134a as the test medium. The baseline configuration with protuberances, Configuration \#2, is shown in Fig. 23. 


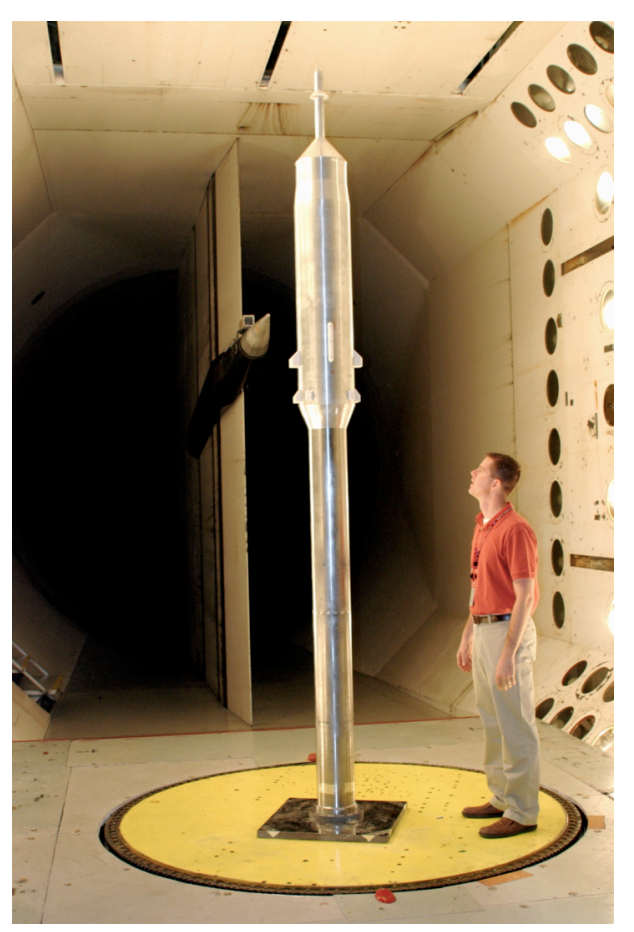

Figure 23. GWL CM Configuration \#2, baseline w/protuberances (flow azimuth $=0^{\circ}$ ) - looking downstream.

The Ares I CLV was to have a cryogenic upper stage that, if insulated in a manner similar to the Space Shuttle external tanks, would have a surface considerably rougher than that simulated by the upper stage surface finish for baseline model configuration. In addition, previous experimental efforts have indicated that vortex-shedding characteristics can be significantly affected by surface roughness. Therefore, \#36 grit (CAMI) was applied to the upper stage of the model for comparison to earlier test configurations. Although grit size and application was far from optimal, the grit was applied as uniformly as practical to double back tape and then the tape applied to the model. Data was acquired using air at near atmospheric pressure and R-134a at various pressures as the test medium. The baseline configuration with protuberances and upper stage grit, Configuration \#3, is shown in Fig. 24. 


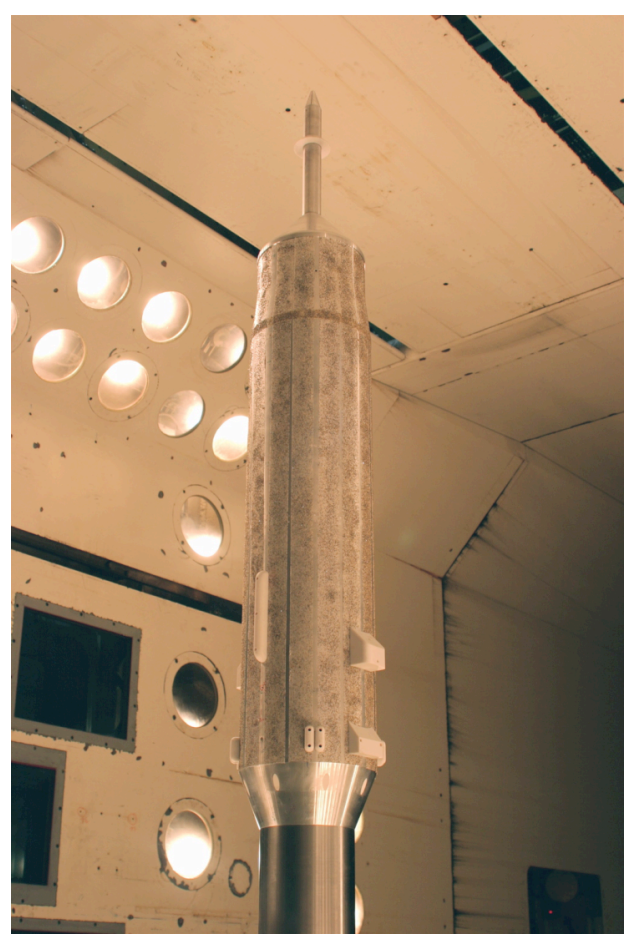

Figure 24. GWL CM Configuration \#3 - baseline model with protuberances and upper stage grit.

The final configuration tested, Configuration \#4, was the baseline configuration with upper stage grit using R$134 \mathrm{a}$ as the test medium. As for the prior runs, this was to investigate the effects of Reynolds number and upperstage surface roughness on the baseline configuration without the protuberances installed. Configuration \#4 was tested using air at near atmospheric pressure and R-134a at various pressures as the test medium and is shown in Fig. 25.

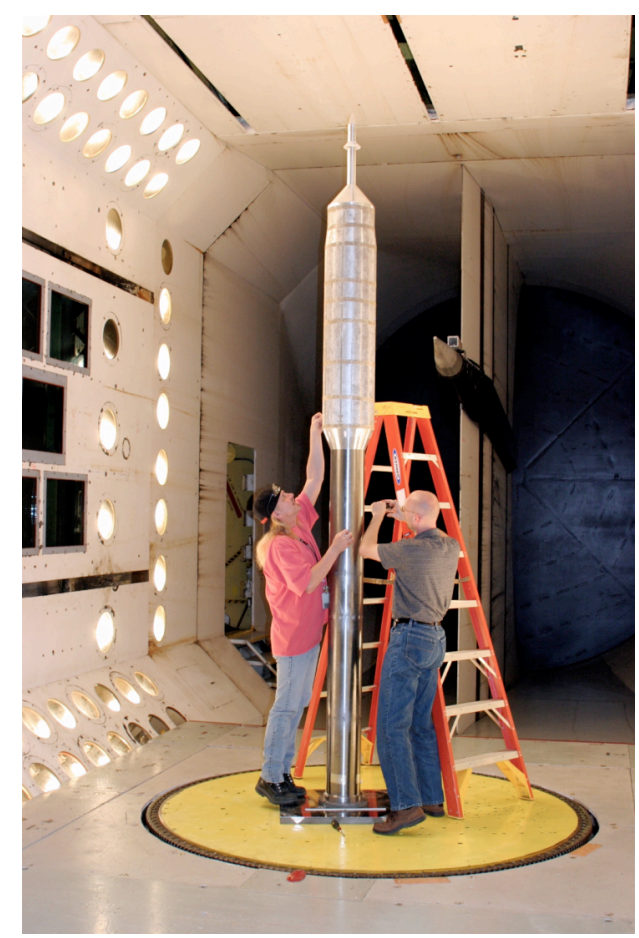

Figure 25. GWL CM Configuration \#4 - baseline (no protuberances) with upper stage grit. 


\section{B. Ares I-X Ground Wind Loads Model}

\section{General Description}

The launch vehicle portion of the Ares I-X GWL wind-tunnel model was an approximately four percent lengthscaled, dynamically-aeroelastically scaled model of the Ares I-X FTV. The launch-vehicle model possessed the appropriate scaled geometry, running mass and stiffness, natural frequencies and mode shapes for critical modes, and all significant protuberances as defined by available geometry and FEM's in early 2008. The launch vehicle model instrumentation was determined by the requirement to get accurate steady and dynamic loads and response (accelerometer) data for loads analysis as well as steady and unsteady pressure data to assist in understanding any dynamic phenomena that occurred during wind-tunnel testing. The Ares I-X GWL model also included a mobile launch platform (MLP) simulator constructed for aerodynamic influence. The MLP simulator was not elastically scaled, did not make contact with the launch vehicle portion of the wind tunnel model, and was not instrumented. In addition, a fixed service structure (FSS) tower and rotating service structure (RSS) were also constructed to simulate the aerodynamic influence of these KSC LC-39B structures. Similar to the MLP, the FSS and RSS components were not instrumented, elastically scaled or connected to the launch vehicle portion of themodel. The OML of the MLP, FSS, and RSS were based on CAD models of these structures and simplification of these was determined by technical requirements and engineering judgment, as well as cost and schedule constraints. Finally, a pedestal balance was designed and constructed to simulate the asymmetric stiffness at the base of the Ares I-X FTV that results from the aft skirt attachment points and MLP hold-down posts. For the wind tunnel model, the pedestal balance served as the attachment between the launch-vehicle model and GWL TT and was instrumented to directly measure the base bending moments and forces. The Ares I-X GWL model was designed to simulate the Ares I-X FTV during rollout to and on-pad stay at the KSC LC-39B. Photos of the Ares I-X FTV during rollout and on pad at KSC LC-39B are presented in Fig. 26 and and Fig. 27, respectively.

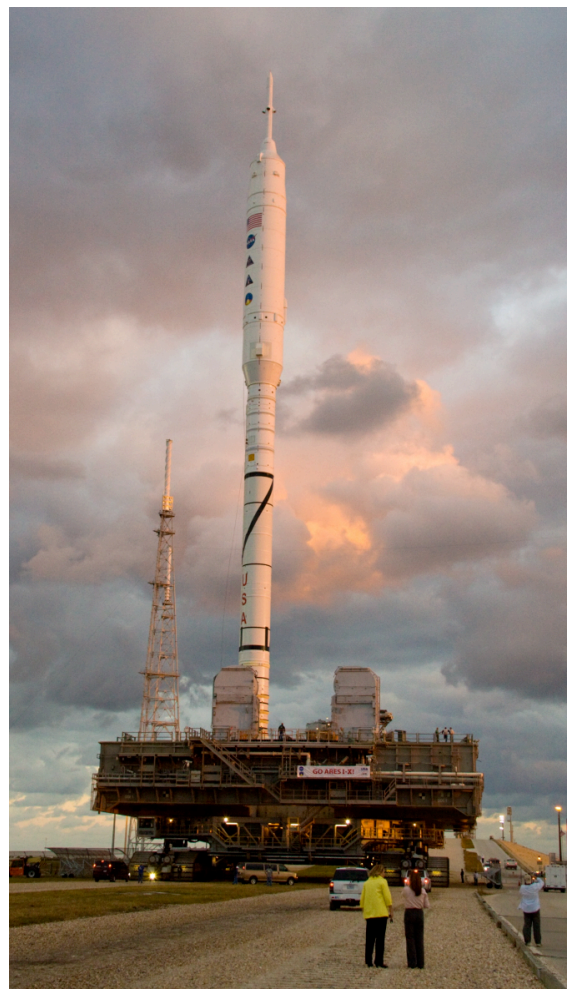

Figure 26. Ares I-X FTV on MLP during rollout to KSC LC-39B. 


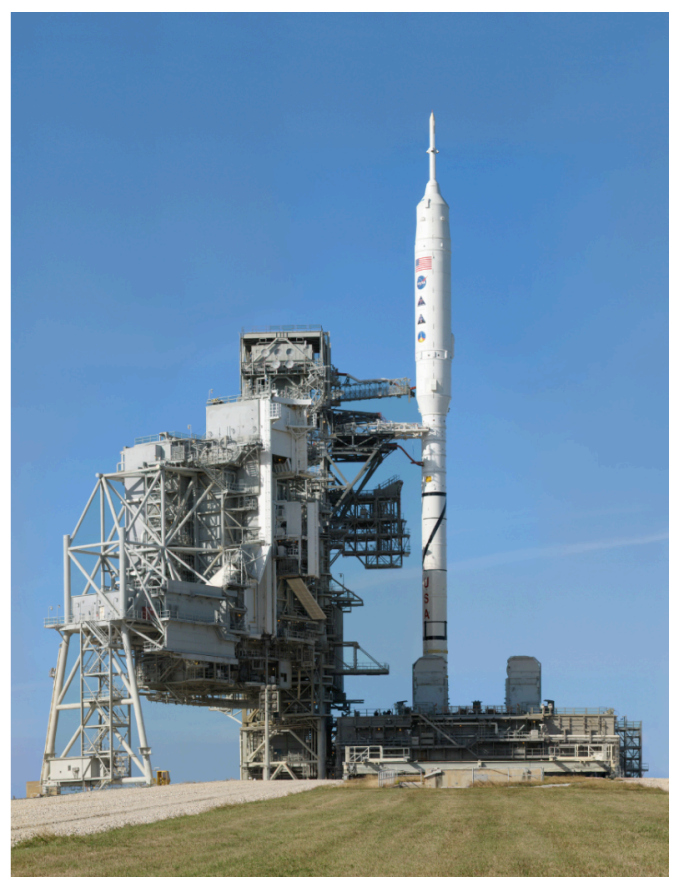

Figure 27. Ares I-X FTV on MLP at KSC LC-39B.

\section{Launch Vehicle Model}

Similar to the GWL CM, the Ares I-X GWL model geometry scale factor of 4.05 pecent was selected based on the maximum height of the launch vehicle model, approximately 13 feet, that could be tested in the TDT with minimal estimated test section wall (ceiling) effects. The OML selected for the launch vehicle model was the Ares I-X FTV OML definition as defined in November 2007. Ares I-X FTV reference layout is presented in Fig. 28. A solid model representation of the Ares I-X FTV configuration with protuberances highlighted in red is shown in Fig. 29. The Ares I-X FTV model structural body coordinate system was selected to be the same as used for the fullscale vehicle. The orientation of the launch vehicle model coordinate system with relation to the KSC LC-39B model hardware was also selected to be identical to that of the full-scale vehicle. The Ares I-X FTV structural body coordinate system and clocking are presented in Fig. 30 and Fig. 31, respectively. The Ares I-X FTV systems tunnels and the LH2 feedline on the upper stage were determined to be potentially very critical in affecting vortexshedding characteristics on the model. These protrusions along with other significant protuberances were included on the launch vehicle model. There was no requirement to simulate the surface roughness of the Ares I-X FTV, beyond inclusion of protuberances, except to better simulate full-scale diametral Reynolds number and flowtransition characteristics. The application of fine surface grit to the entire vehicle model was determined to be required for this purpose and is discussed later in this paper.

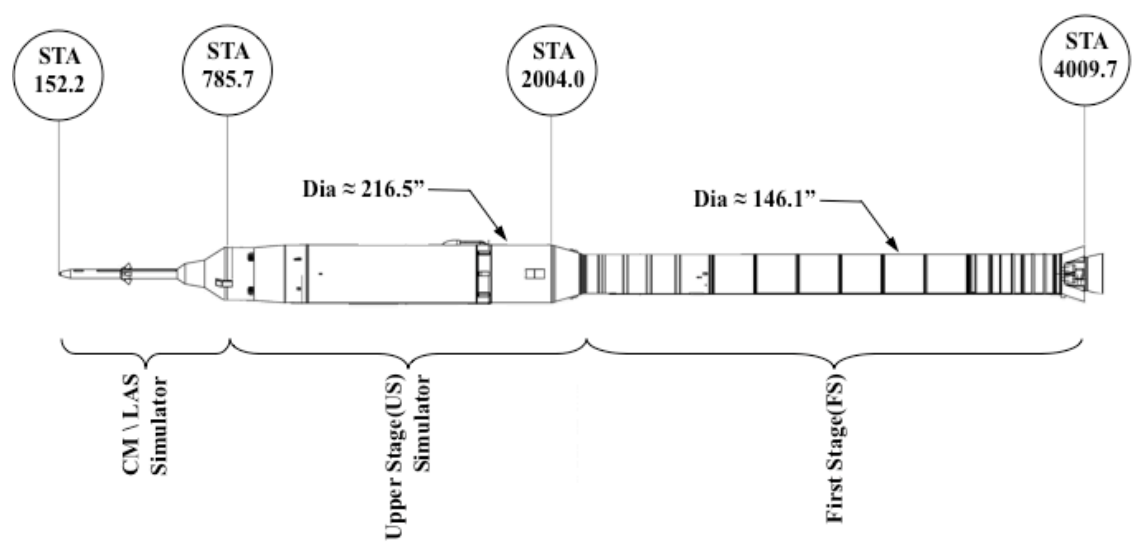

Figure 28. Ares I-X FTV configuration details (full-scale).

American Institute of Aeronautics and Astronautics 


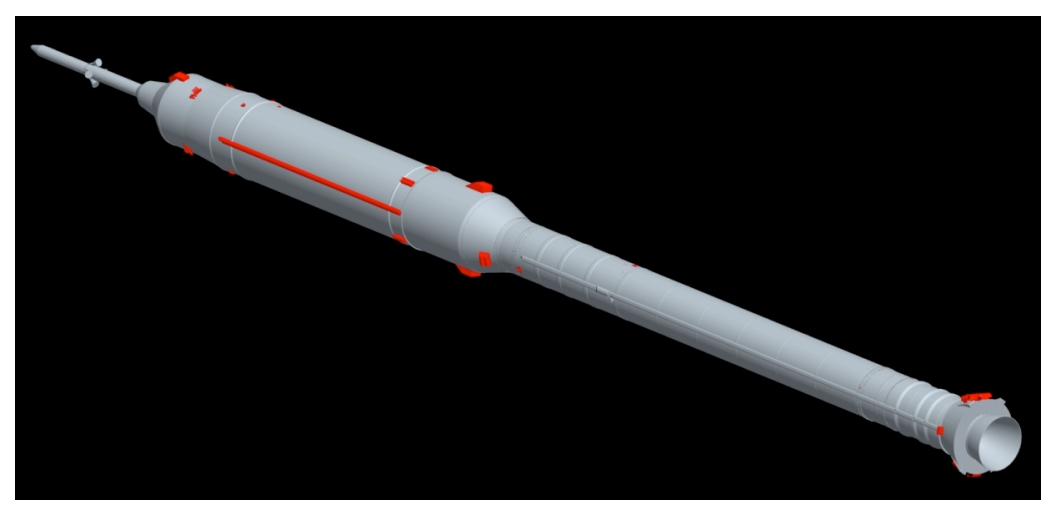

Figure 29. Ares I-X FTV solid geometry, protuberances highlighted in red.

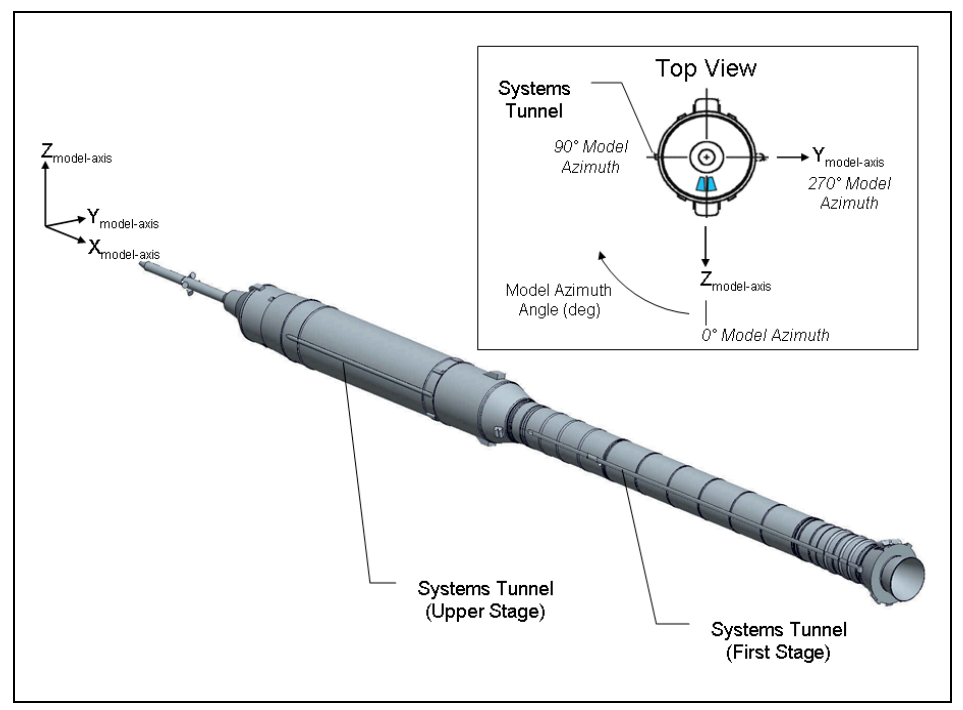

Figure 30. Ares I-X FTV model-axes coordinate system and model-azimuth angle definition.

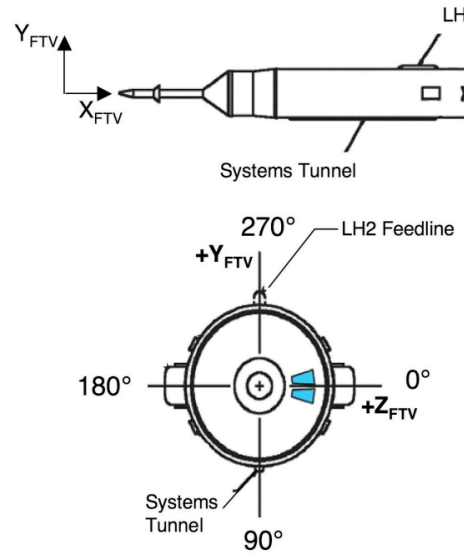

VIEW LOOKING AFT

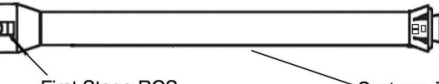

First Stage RCS Systems Tunne

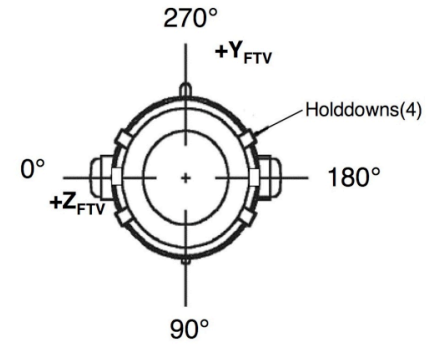

VIEW LOOKING FWD

Figure 31. Ares I-X FTV clocking.

The Ares I-X GWL model launch vehicle model structure was based significantly on lessons learned from the GWL Checkout Model project in that the model was constructed of several metal tube sections of varying thickness to simulate the scaled running stiffness of the Ares I-X FTV. Joints between tube sections were designed to minimize inherent structural damping. The outer surface of the tube sections formed the primary OML with 
attachment points for protuberances. Interior attachment points were located as required for instrumentation, ballast weight, and damper installation. The Ares I-X FTV model, and all other model components, were required to be designed to withstand predicted steady drag and dynamic lift loads in any direction at a planned maximum test dynamic pressure of 150 psf.

Based on data requirements, previous GWL models, and a desire for a simple launch vehicle model base design, the Ares I-X FTV model was designed to be cantilever mounted to the top of a then undefined balance.

The launch vehicle model was dynamically-aeroelastically scaled from the full-scale Ares I-X FTV, in a manner similar to the GWL CM, using standard dynamic-aeroelastic scaling laws with the exception that Mach number was unity scaled (flow assumed to be quasi-incompressible over the range of interest). Full-scale and model-scale flow conditions for scaling were chosen based on Ares I-X FTV wind requirements and wind speeds that were considered critical for $2^{\text {nd }}$ bending mode WIO. The launch vehicle wind-tunnel model was designed to match, as closely as practical, the scaled mode shapes and frequencies for the first four primary vehicle modes of natural vibration, first and second bending in the $\mathrm{Y}$ and $\mathrm{Z}$ axes, with emphasis on the two second bending modes. Higher modes were not considered to be important for launch-vehicle GWL and wind induced oscillation (WIO) characteristics. Model mass and stiffness characteristics were based on those available in November 2007 in the form of NASTRAN finite element models (FEMs) of the full-scale Ares I-X FTV on the MLP. The primary FEM used for design of the Ares I-X FTV model is presented in Fig. 32.

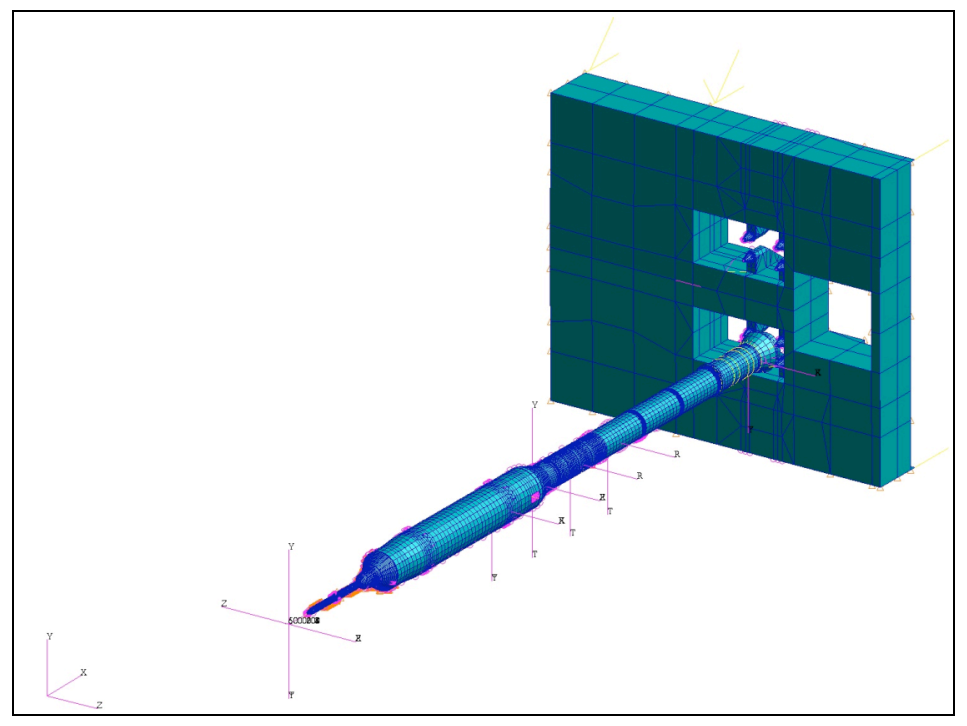

Figure 32. NASTRAN finite element model for Ares I-X on MLP.

The Ares I-X FTV FEM was used to generate mode shapes and frequencies of the first four vehicle bending modes for the vehicle on the MLP. These mode shapes and frequencies were later used for comparison to, and evaluation of, the wind-tunnel model design and later to modal results from the actual wind-tunnel model. Modal frequencies and corresponding node point locations, especially for modes 3 and 4, were used to quickly evaluate similitude of the Ares I-X GWL wind-tunnel model design to the full-scale vehicle. Ares I-X FTV full-scale FEM modal results for critical vibration modes are presented in Table 7.

Table 7. Full-scale vibration modes for Ares I-X FTV FEM.

\begin{tabular}{|c|c|c|c|}
\hline Mode \# & Mode Description & Freq, Hz & Node Point Loc.(s) \\
\hline 1 & $1^{\text {st }}$ bending, Y-dir. & 0.17 & $\approx$ Base of Vehicle \\
\hline 2 & $1^{\text {st }}$ bending, Z-dir. & 0.21 & $\approx$ Base of Vehicle \\
\hline 3 & $2^{\text {nd }}$ bending, Y-dir. & 1.02 & Base and STA 1765.4” \\
\hline 4 & $2^{\text {nd }}$ bending, Z-dir. & 1.16 & Base and STA 1691.4” \\
\hline
\end{tabular}

The mobile launch platform was removed from the full-scale Ares I-X FTV FEM and the vehicle base modeled as fully constrained (cantilevered) for the calculation of mode shapes and frequencies. This was done since the launch vehicle wind-tunnel model was initially designed as cantilevered at its base. The frequencies and 
corresponding node point locations of the first four full-scale modes of vibration of the Ares I-X FTV cantilevered at the base of the vehicle aft skirt are presented in Table 8. The differences in the results presented in Table 7 and Table 8 illustrate the significant effect of the MLP and hold-down posts on the critical vibration modes for the Ares I-X FTV.

Table 8. Full-scale vibration modes for Ares I-X FTV FEM cantilevered at base of aft skirt.

\begin{tabular}{|c|c|c|c|}
\hline Mode \# & Mode Description & Freq, Hz & Node Point Loc.(s) \\
\hline 1 & $1^{\text {st }}$ bending, Y-dir. & 0.22 & $\approx$ Base of Vehicle \\
\hline 2 & $1^{\text {st }}$ bending, Z-dir. & 0.23 & $\approx$ Base of Vehicle \\
\hline 3 & $2^{\text {nd }}$ bending, Y-dir. & 1.23 & Base \& STA 1670 \\
\hline 4 & $2^{\text {nd }}$ bending, Z-dir. & 1.24 & Base \& STA 1662 \\
\hline
\end{tabular}

As for the GWL CM, a standard dynamic-aeroelastic scaling was performed for the Ares I-X FTV that resulted in a set of full-scale to model-scale factors being generated that were used to scale the Ares I-X FTV geometry and FEM derived information to use as a basis for launch vehicle model design. Based on the GWL Checkout Model project results and preliminary Ares I-X FTV model design activities, the I-X launch vehicle model did not follow the full-scale Reynolds number and reduced frequency curve for the Ares I-X FTV, similar to that presented in Fig. 20. In addition, due to aerodynamic similitude requirements, the diameter for the Ares I-X FTV model was scaled using the length scale factor instead of a larger value as was done for the GWL Checkout Model. As a result, Ares I-X GWL model wind-tunnel test Reynolds numbers were approximately $30 \%$ of corresponding full-scale vehicle values.

Based upon fluid and geometric parameters similar to those used for the GWL CM, the Ares I-X FTV predicted structural characteristics, and an assumed critical Strouhal number of 0.20 for the Ares I-X FTV (2 ${ }^{\text {nd }}$ mode WIO, upper stage region vortex shedding), the required full-scale and model-scale parameters for calculating dynamicaeroelastic scale ratios were determined. Full-scale values for vehicle length, air density, air viscosity, air sonic velocity, and an assumed critical Strouhal number of 0.20 resulted in full-scale design values of wind speed and Reynolds number, for upper stage vortex shedding induced WIO, of 98.3 feet per second (58.3 knots) and 11.29 million, respectively. Ares I-X launch vehicle model values for length, test medium properties, model Strouhal number of 0.20 (assumed), and a test Mach number limit (although test data was acquired at higher values) and Reynolds number target of 0.30 and 3.25 million, respectively, resulted in the calculation of the scale factors presented in Table 9.

Table 9. Ares I-X FTV model design scale factors.

\begin{tabular}{|l|c|}
\hline \multicolumn{1}{|c|}{ Name } & Scale Factor \\
\hline Length & 0.0405 \\
\hline Velocity & 1.59 \\
\hline Strouhal number & 1.00 \\
\hline Frequency & 39.25 \\
\hline Weight (mass) & $2.01 \times 10^{-4}$ \\
\hline Reynolds number & 0.288 \\
\hline
\end{tabular}

The final value for model-design Reynolds number for $2^{\text {nd }}$ mode WIO was selected to correspond to a test section Mach number of slightly below 0.30 to allow for as-tested model aerodynamic and structural uncertainties. The assumption of Strouhal number similitude between the full-scale vehicle and wind-tunnel launch vehicle model resulted in the calculation of the remaining scale factors required for design of the wind-tunnel model. Application of these scale factors to full-scale vehicle parameters and characteristics resulted in the wind-tunnel model having a target weight of approximately 370 pounds and an average second bending modes ( $\mathrm{Y}$ and $\mathrm{Z}$ axes) frequency of 42.8 $\mathrm{Hz}$. Corresponding predicted model test conditions for $2^{\text {nd }}$ bending mode, upper stage WIO, were $156.3 \mathrm{ft} / \mathrm{s}, 88 \mathrm{psf}$, and 0.284 for velocity, dynamic pressure, and Mach number, respectively.

The relatively low value of Reynolds number scale ratio, 0.288 , was a significant concern even though the predicted value of Reynolds number for upper stage vortex shedding induced WIO for the wind-tunnel model was thought to be high enough, greater than 2.5 to 3.0 million, to ensure that the boundary layer would be fully turbulent 
and similar to the flow expected on the upper stage of the full-scale vehicle. The first stage diameter, however, was approximately $2 / 3$ that of the upper stage with a corresponding lower Reynolds number for planned test conditions. Model first-stage vortex shedding characteristics might therefore not be similar to those on the full-scale vehicle with a corresponding negative effect on similitude for first stage $2^{\text {nd }}$ bending mode WIO test data. Low Reynolds number ratio was also a concern with regard to acquisition of accurate steady load (lift and drag) and moment data. As a result, the surface roughness of the Ares I-X FTV model was increased to simulate diametral Reynolds numbers representative of that expected for the full-scale vehicle ${ }^{5}$. The selection of grit size was determined by the requirement that the grit be large enough to cause proper flow transition along the length of the launch vehicle model but not so large as to disrupt the vortex shedding. In addition, selection of too large a grit particle size would cause unrealistically large values of drag to be measured. Based on the results reported in Ref. 8, the effective increase in Reynolds number was calculated as a factor of $\frac{\delta}{3.5 \times 10^{-5} D}$, where $\delta$ was the grit roughness diameter and D is the local cylinder diameter. Since the model-scale to full-scale Reynolds number ratio of the Ares I-X FTV model design for all conditions was approximately 0.29, the desired Reynolds number increase factor was the inverse of 0.29 , or approximately 3.45. For a first stage diameter of 5.92 inches, this equated to a desired $\delta$ of approximately 0.0007 inches. For an upper stage diameter of 8.77 inches, this equated to a desired $\delta$ of approximately 0.0011 inches. As a result, a grit size of 400 was chosen for the first stage with an average grit particle diameter of 0.00092 inches, and a grit size of 360 was chosen for the upper stage and service module area with an average grit particle diameter of 0.00112 inches. Grit specifications were based on those supplied by the manufacturer and standards established by CAMI. A grit size of 360 was also used for the frustum and command module areas of the model. No grit was applied to the LAS tower region. The region where each type of grit was applied to the vehicle model is illustrated in Fig. 33.

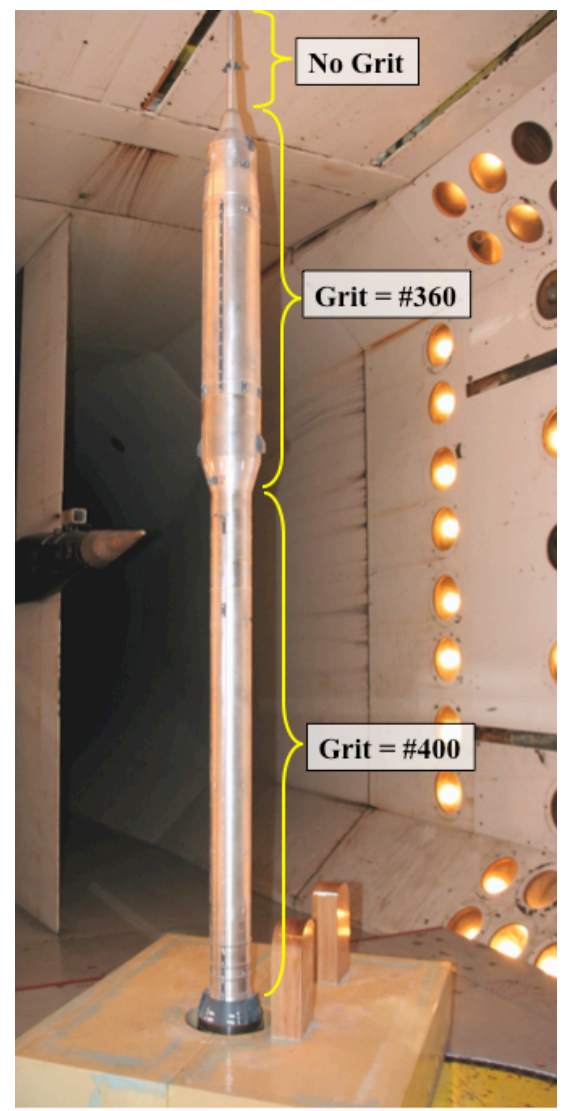

Figure 33. Ares I-X FTV model grit layout. 
The full-scale and final model-scale design reduced frequency-Reynolds number curves are presented in Fig. 34. The figure also presents model-scale velocities at selected points for comparison to full-scale ground-wind velocities, uniform flow assumed, for the Ares I-X FTV.

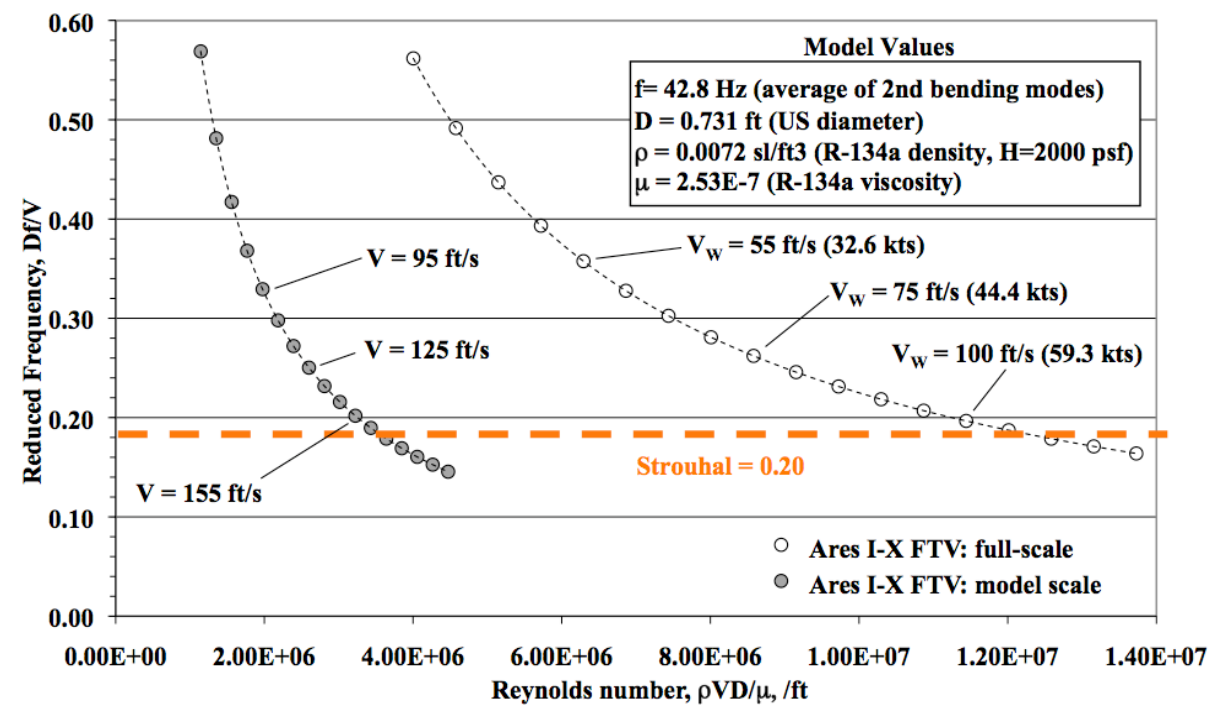

Figure 34. Reduced freq. vs Re for Ares I-X FTV, full-scale and model-scale.

Launch-vehicle model design involved using scaled design values for the Ares I-X FTV OML, and stiffness and mass distributions, to develop the I-X launch vehicle model structure and ballast weights using classical and finite element analysis based design methods. The number of ballast weights was minimized for ease of manufacture and assembly without negatively impacting model dynamic characteristics. A final ballast weight configuration of eight tungsten weights was selected. Each ballast weight was secured to the inside of the launch vehicle model at a specified location using an aluminum spider bracket and four steel shear pins. The weight and location of the ballast weights is presented in Table 10. The final design of the model was checked for proper dynamic characteristics using finite element analysis and was found that the two critical $2^{\text {nd }}$ bending modes were in good agreement with design objectives for frequency and mode shapes. Model design was also in good agreement with scaled vehicle mass and center of gravity location.

Table 10. Ares I-X model final design ballast weight details.

\begin{tabular}{|c|c|c|c|}
\hline Ballast Wt. \# & Weight, lbs & Model Station, in & Stage Location \\
\hline 1 & 7.0 & 42.85 & Upper \\
\hline 2 & 14.0 & 70.91 & Upper \\
\hline 3 & 9.0 & 105.43 & First \\
\hline 4 & 48.0 & 115.38 & First \\
\hline 5 & 48.0 & 128.42 & First \\
\hline 6 & 25.0 & 138.14 & First \\
\hline 7 & 34.0 & 146.47 & First \\
\hline 8 & 26.0 & 155.62 & First \\
\hline
\end{tabular}

As stated earlier, structural damping is a critical parameter in determining the response of an exposed launch vehicle to the periodic lifting forces caused by vortex shedding, especially when the shedding occurs at or near the frequency of a critical mode of vibration of the vehicle during rollout or on-pad stay. Past wind-tunnel studies and analysis efforts have demonstrated that variations in inherent vehicle damping as small as $0.5 \% \mathrm{C} / \mathrm{Cc}$ can result in significant increases or decreases in vehicle response during WIO. As a result, the Ares I-X FTV model was purposely designed to have low inherent, i.e. worst case, structural damping, that could be increased to as high as $2 \% \mathrm{C} / \mathrm{Cc}$ or greater through the use of an internal tunable (variable) damper mechanism. 
The damper concept developed for the Ares I-X GWL model test employed a tuned mass-spring-damper concept and was successfully used to increase damping of the roll-out configuration late in the wind-tunnel test. The tuned mass-spring-damper was installed internally near the top of the vehicle model upper stage and, during testing, was installed in place of ballast weight \#1. The weight of the damper was very close to the weight of ballast weight \#1 or approximately 7 pounds, depending on the number of tuning weights installed on the damper. This tuned damper utilized a moving mass constrained by a linear slider and compression springs. Damping was provided by a rotary dashpot and rack gear. The slider mass, spring rates, and dashpot viscosity determined the mode of vibration affected and the amount of damping added to the vehicle for that specific mode. The amount of moving mass and/or the spring rates was adjusted by adding tuning weights and/or installing different springs, respectively. There were two damper systems on the damper unit and these were oriented $90^{\circ}$ apart. These two linear degrees of freedom for the damper unit were fixed to align with the fundamental vehicle model axes, $\mathrm{Y}$ and $\mathrm{Z}$, when installed in the model and each was tuned to increase damping for a specific fundamental mode with minimal impact on any other critical modes of vibration. Photos of the damper prior to installation in the Ares I-X FTV model, are presented in Fig. 35.

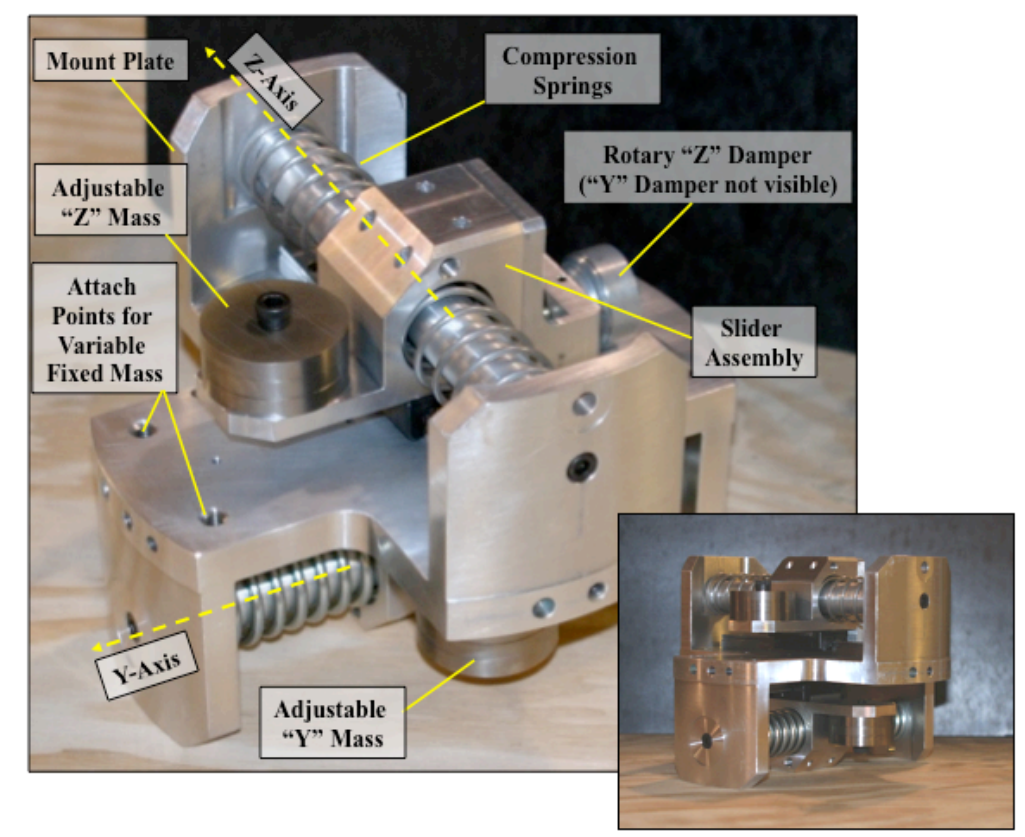

Figure 35. Tuned mass-spring-damper assembled prior to wind-tunnel test.

Ares I-X launch vehicle model instrumentation was similar to that used on the GWL CM with the exception that the base bending moment data was acquired using a separate balance instead of strain gauge bridges installed on the launch vehicle model structure and there were no steady static pressure orifices. Instead all pressure data was acquired using unsteady pressure transducers.

Two vertical rows of nine piezoelectric-type accelerometers mounted on the $0^{\circ}$ and $90^{\circ}$ model azimuths distributed evenly along the length of the vehicle model were employed to acquire model dynamic response data.

Eight miniature, high-sensitivity piezoresistive unsteady pressure transducers were mounted in-situ at two model stations, 33.89 and 67.50 , both in the upper stage. These pressure transducers were evenly spaced around the circumference of the launch vehicle model at each of the model stations for a total of sixteen model unsteady pressure transducers with an integral orifice at each location. The unsteady pressure transducers were used to acquire steady and unsteady static-pressure data. The pressure transducers were calibrated after installation in the model using a reference pressure tube located at the rear of each transducer.

Installation of Ares I-X FTV model instrumentation and wiring during launch-vehicle model assembly is shown in Fig. 36. The approximate location of the accelerometers and pressure transducer model stations, along with the location of the damper unit (when installed) is shown on the Ares I-X FTV model in the TDT test section in Fig. 37. 


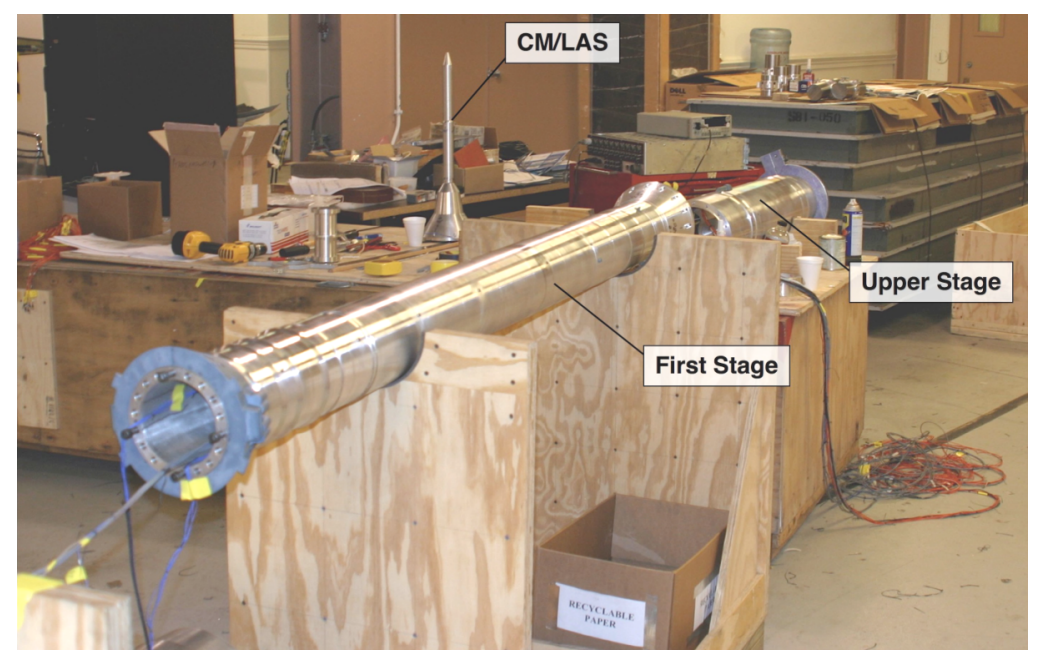

Figure 36. Ares I-X FTV model during assembly and installation of instrumentation.

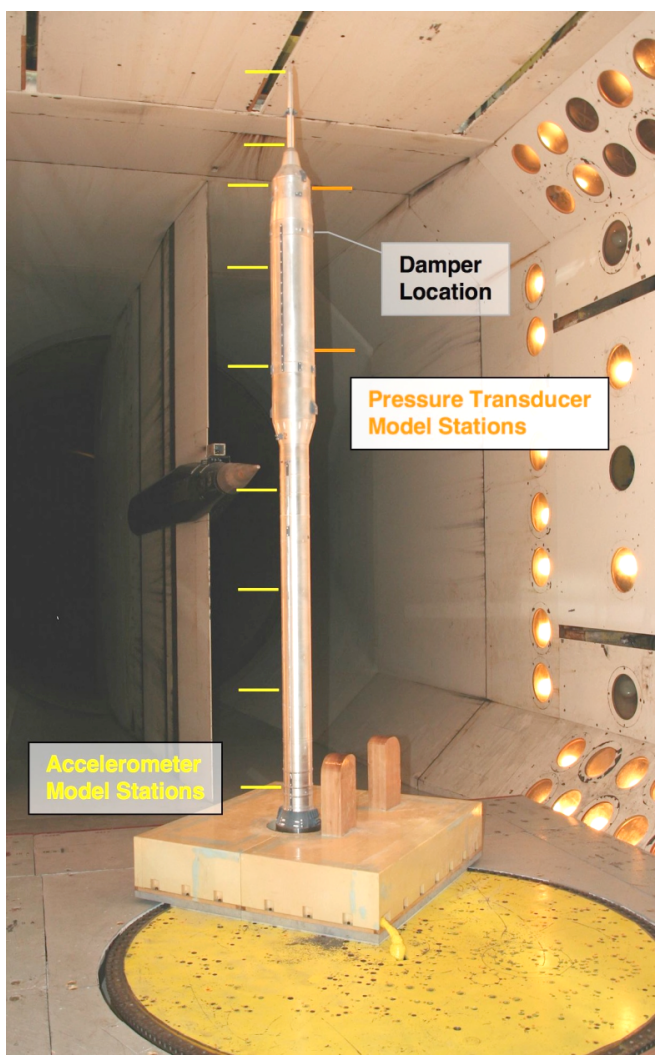

Figure 37. Ares I-X FTV model instrumentation and damper model stations.

\section{Pedestal/Balance}

The balance used in the Ares I-X GWL model test, and sometimes referred to during the project as the pedestal or pedestal balance, was a four-component balance that was intended to directly measure vehicle model forces in, and base bending moments about, the $\mathrm{Y}$ and $\mathrm{Z}$ model axes. This data would be converted to full-scale to calculate launch vehicle model lift and drag forces and bending moments based on flow angle (wind azimuth angle). Accurate force data would also enable calculation of center of pressure on the launch vehicle model, useful for correlation to computational results and understanding of model steady and unsteady flow characteristics during testing. The balance also simulated the scaled asymmetric stiffness at the base of the full-scale Ares I-X Flight Test Vehicle that resulted from the aft skirt attachment points and MLP hold-down post stiffness and geometry. Finally, 
the balance served as the mounting interface between the base of the vehicle model and the TDT GWL TT and resided inside, and not in contact with, the model component that simulated the full-scale MLP.

The geometry and OML for the balance were determined by the design of the Ares I-X FTV model and the MLP that surrounded and shielded the balance during testing. Launch vehicle model wiring and tubing umbilicals passed through the center of the balance and out to the TDT east wall instrumentation patch panel. The height of the balance was 10 inches due to limits on the total height of the launch vehicle model on the balance in the TDT test section.

The combined requirements for balance stiffness, strength, and OML caused concern with regard to accuracy of the balance, especially for measurement of forces in the $\mathrm{Y}$ and $\mathrm{Z}$ directions. Balance load checks performed at NASA during ground testing, prior to the wind-tunnel test, discovered that accuracy of the balance in measuring forces, $F_{Y}$ and $F_{Z}$, was poor when moments and forces were applied in combination. The calibration and checkload results indicated that the balance was accurate to within $\pm 1 \%$ of applied bending moment regardless of location, direction, and application point of the load, while the accuracy in measuring forces was so poor that no force data was able to be acquired during the wind-tunnel test.

A dynamic load was also applied to the balance in the $\mathrm{Y}$-axis and $\mathrm{Z}$-axis directions to determine if the balance output would be accurate for loads at frequencies up to $100 \mathrm{~Hz}$. This dynamic load check was performed without a model installed on the balance. A large electromagnetic shaker was used to apply loads at specific frequencies and over the range of interest, 0 to $100 \mathrm{~Hz}$, using sine sweeps and noise as input. The shaker was rigidly mounted to a floor strongback, located at the TDT, during the dynamic load test. Applied load was measured using a calibrated dynamic load cell placed between the shaker and the balance. The load was input into the balance through a small threaded plate that was bonded to the side of the top flange of the balance. Test data indicated that the balance moment data was accurate, from 0 to $100 \mathrm{~Hz}$, using static load calibration coefficients. No further testing of this type was performed in the TDT test section.

A photo of the balance just after delivery to NASA and a photo of the balance installed in the TDT test section with the Ares I-X FTV model on the GWL TT are presented in Fig. 38 and Fig. 39, respectively.

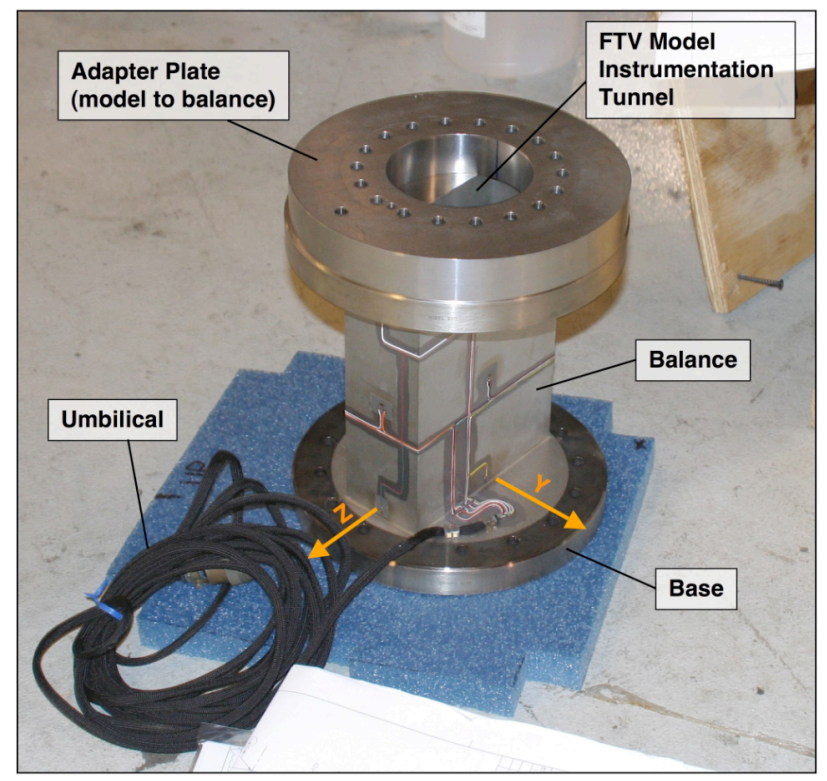

Figure 38. Ares I-X GWL model balance with adapter plate. 


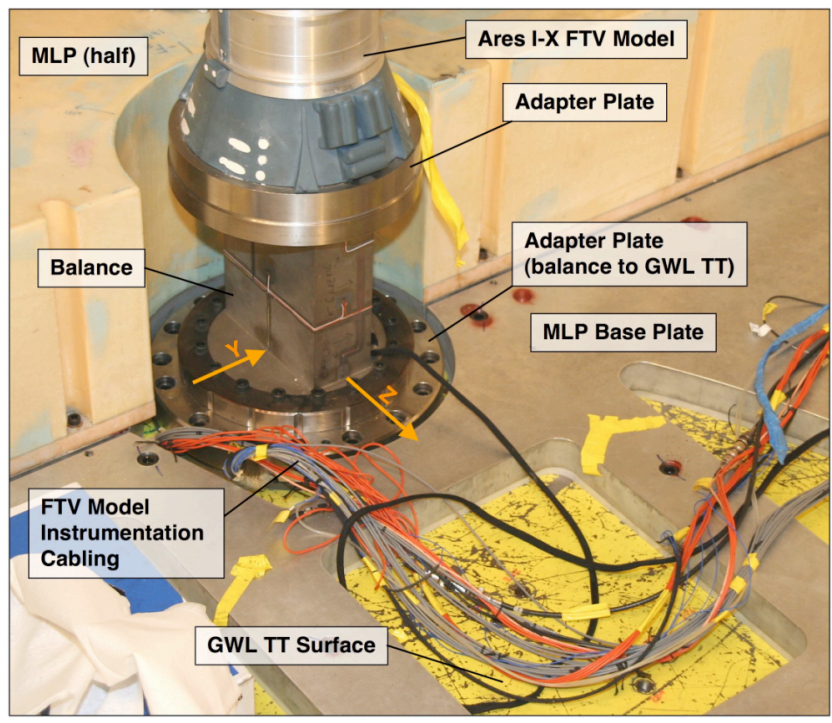

Figure 39. Ares I-X GWL model balance installation in TDT. 40 .

The direction of positive forces and moments in the vehicle model-axis coordinate system are depicted in Fig.

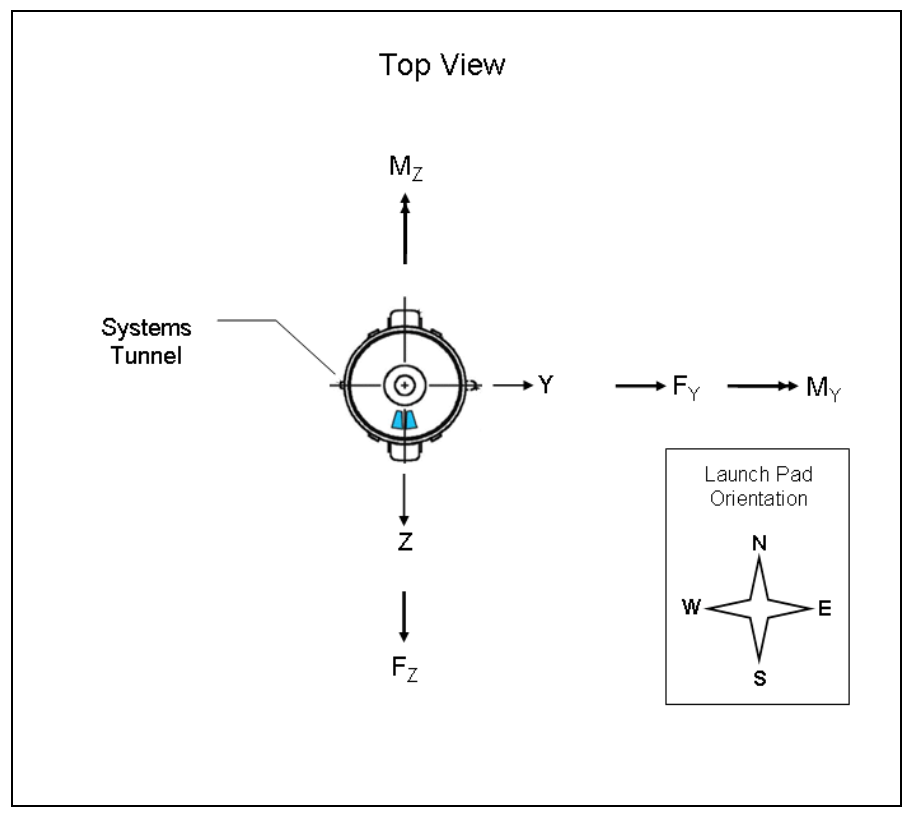

Figure 40. Definition of positive foorces and moments in vehicle model-axis system.

The wind-axis coordinate system along with the definition of positive drag and lift forces and resulting moments for the launch vehicle model are presented in Fig. 41. The moments are defined such that a positive drag force results in a positive base bending moment due to drag (DBM) and a positive lift force results in a positive bending moment due to lift (LBM).

In the TDT test section, the direction of flow is fixed. Rotating the GWL TT with the model mounted on it simulated the variation in wind azimuth angle. Rotating the model counterclockwise (as viewed from above) was analogous to full-scale wind azimuth rotating clockwise. GWL TT angle (THETATT) was therefore defined, as shown in Fig. 41, as positive counterclockwise so that the value of the turntable angle was equal to full-scale wind azimuth angle. It should be noted that the full-scale vehicle orientation with respect to North during roll-out was different as the vehicle turned during rollout to KSC LC-39B. 


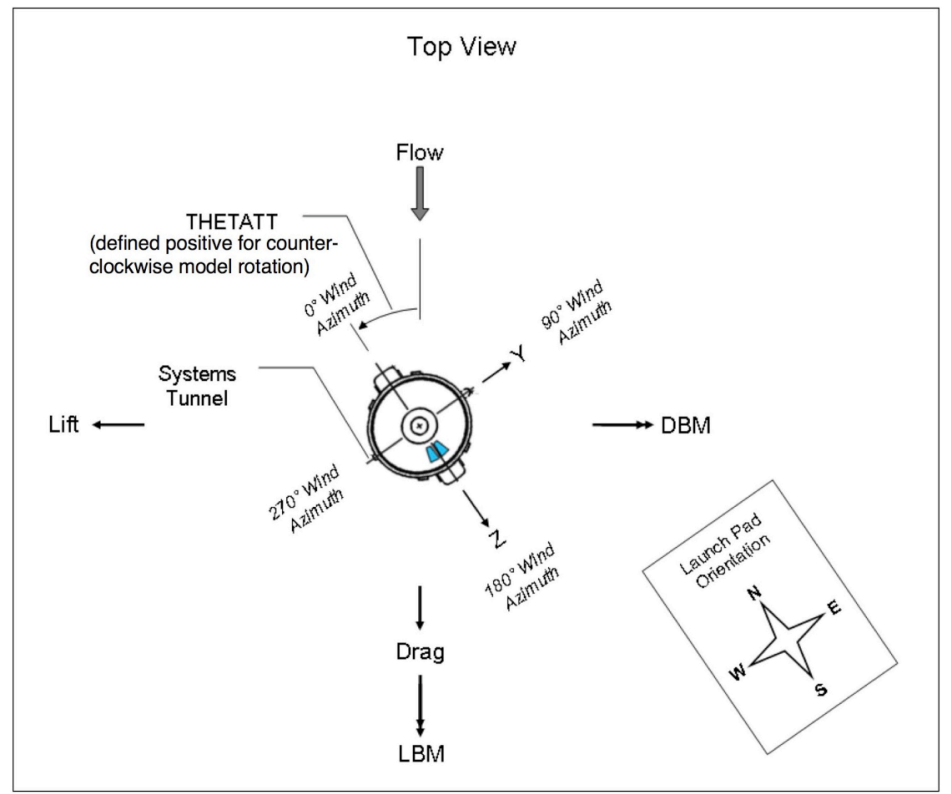

Figure 41. Ares I-X FVT model wind-axis coordinate system and definition of forces and moments.

The final design of the balance was based on normal modes analyses performed using a NASTRAN FEM of the Ares I-X FTV wind-tunnel model attached to a relatively simple beam-element based NASTRAN FEM for the balance and adapter plate. The assembled FEM was cantilevered at the base of the balance, MS 173.64. The frequencies, mode shapes, and node point locations, for $2^{\text {nd }}$ bending modes, were compared to scaled results from the full-scale Ares I-X FTV shell-element NASTRAN FEM where the Ares I-X FTV was on the MLP. It was determined that the predicted dynamic characteristics for the FTV model on the balance were in reasonably good agreement with the full-scale vehicle on the MLP. As-built modal data was not available until ground testing of the hardware could be performed just prior to the wind-tunnel test. The NASTRAN FEM for the Ares I-X FTV windtunnel model on the balance is presented in Fig. 42. Predicted frequencies, mode shapes, and $2^{\text {nd }}$ mode node point locations for the launch vehicle model on the balance are presented, with scaled full-scale target values for comparison in parentheses, in Fig. 43 and Fig. 44.

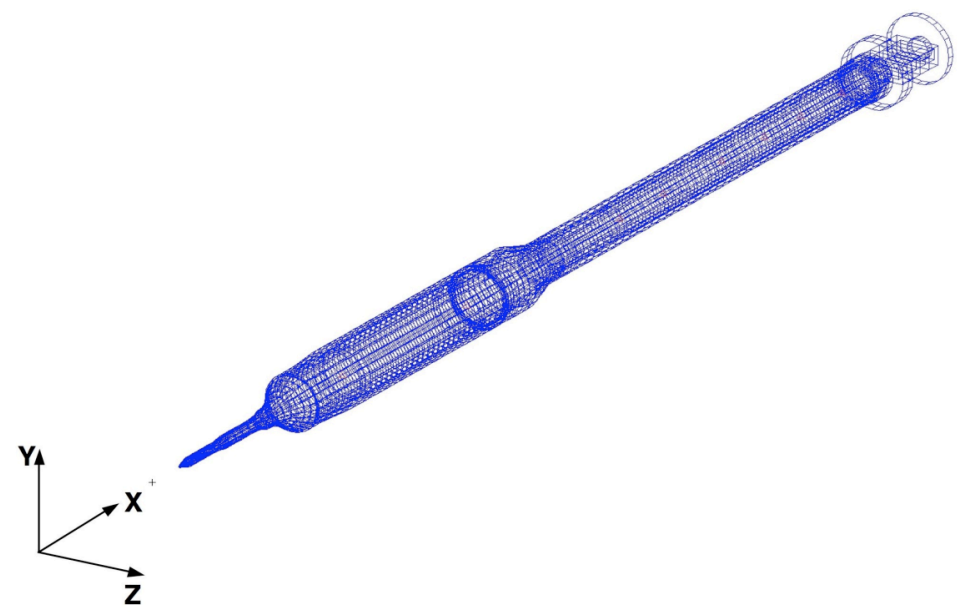

Figure 42. Ares I-X FTV model and balance, NASTRAN FEM. 


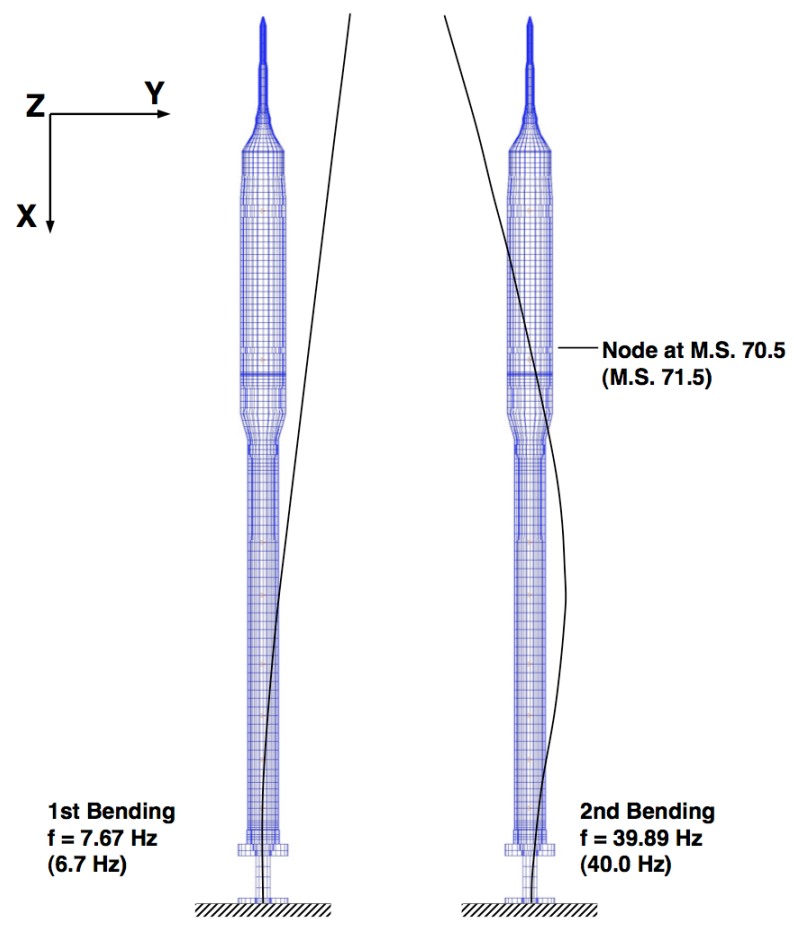

Figure 43. Ares I-X FTV model on balance, NASTRAN FEM, 1st and 2nd bending modes in Y-axis direction.
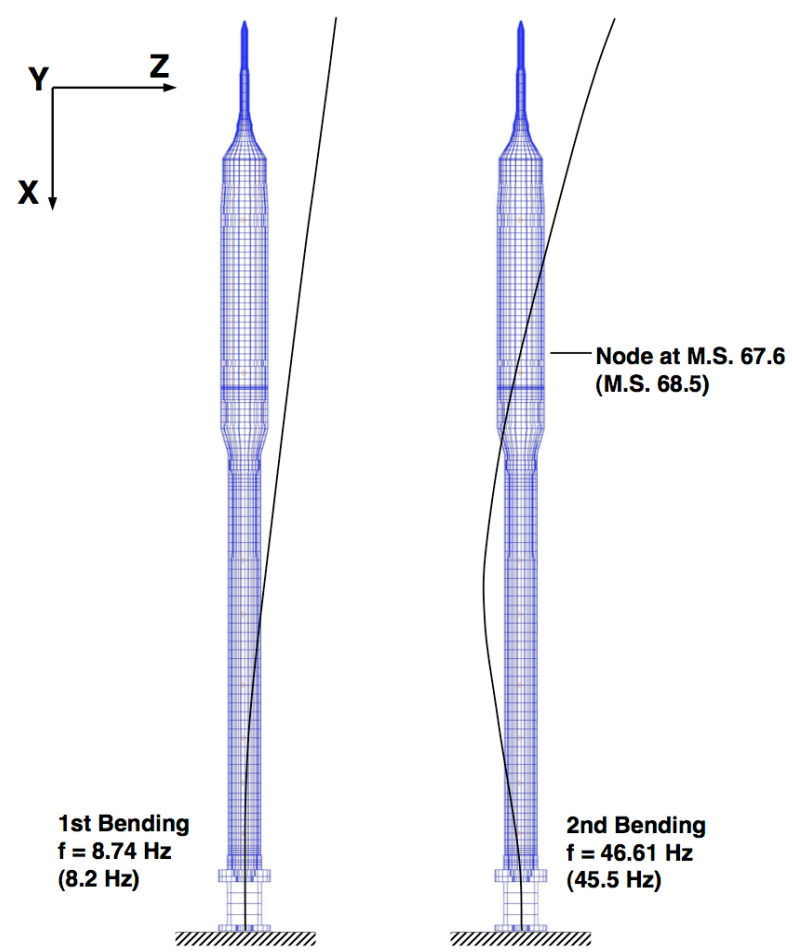

Figure 44. Ares I-X FTV model on balance, NASTRAN FEM, 1st and 2nd bending modes in Z-axis direction.

4. Mobile Launch Platform and Fixed Service and Rotating Service Structures

The Ares I-X GWL model component that simulated the KSC MLP was conceived to be a simple rectangular box of model-scale dimensions properly located relative to the Ares I-X FTV model. The position and shape of the MLP created the basic aerodynamic effects on the vehicle model similar to what would occur on the full-scale vehicle during rollout and on-pad stay and launch configurations. The MLP simulator also served as a shroud to

American Institute of Aeronautics and Astronautics 
protect the balance and instrumentation umbilicals while having no effect on the vehicle model dynamic characteristics. The MLP was securely mounted to the TDT GWL TT and therefore rotated with the other Ares I-X GWL model components during testing.

The model MLP was planned to be of the approximate scaled outer perimeter dimensions of the full-scale MLP with a height sufficient to shield the balance and associated adapter plates. There was no wind flow path under the model MLP due to the desire to limit test section blockage and it was not believed that flow under the model MLP would have improved the simulation of the actual wind flow over and around the full-scale MLP. No additional surface or protuberance details were included except for the inclusion of two tombstone like protuberances to simulate the tail service masts located on the full-scale MLP. A hole in the model MLP at the location of the launch vehicle model existed for placement of the balance with enough clearance to prevent contact between the model MLP and balance at any time during the test. The final size and location of the model MLP was determined by the need to be compatible with those model components simulating the other two primary KSC LC-39B components, the Fixed Service Structure (FSS) and Rotating Service Structure (RSS). This eventually resulted in the dimensions of the model MLP being reduced approximately $32 \%$ and $12 \%$ in the Z-axis and Y-axis, respectively, to allow full rotation of the GWL TT during testing. This reduction in the planform of the model MLP is illustrated in Fig. 45.

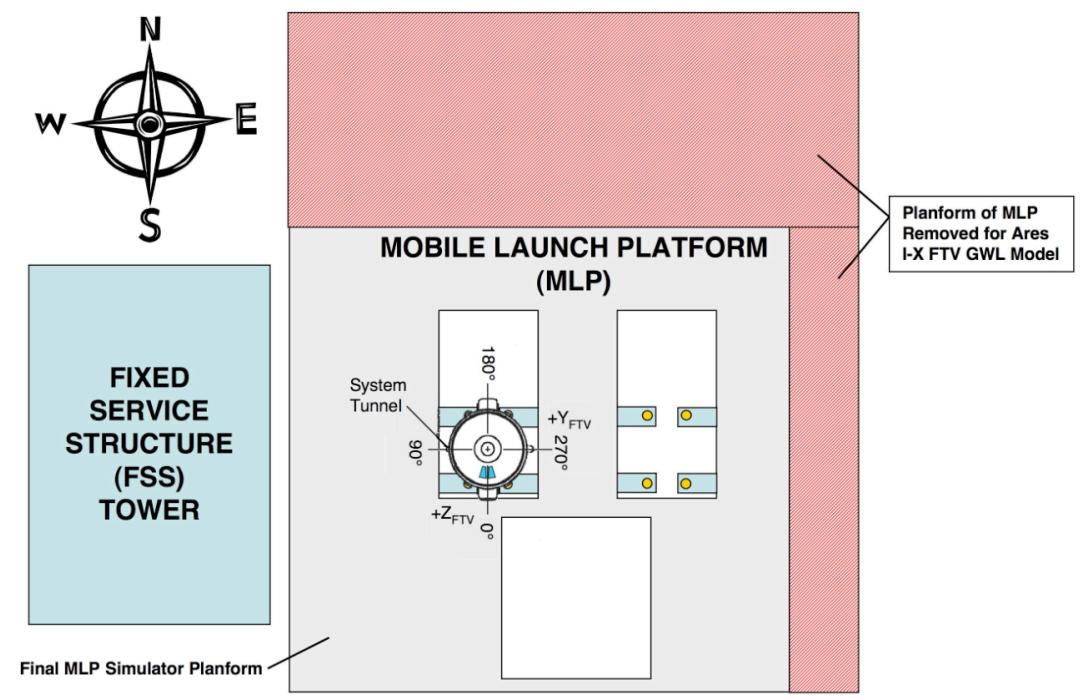

Figure 45. Original and final planform of model MLP used during Ares I-X GWL test.

The model MLP planform was approximately 56.6 inches by 52.5 inches in the model Y and Z axes, respectively and approximately 11.5 inches in height. The model MLP was fabricated in two pieces that were assembled around the balance and launch vehicle model after those had been installed on the GWL TT in the TDT test section. The model MLP had a channel cut into the base of the foam core that was used to route the launch vehicle model and balance instrumentation umbilicals out to the center of the turntable where they were routed down through the spindle of the turntable and to the TDT east wall DAS patch panel. There was no instrumentation on the model MLP. The model MLP was secured to the turntable using bolts and threaded holes that were drilled and tapped into the turntable surface specifically for the model MLP. The model MLP foam core was covered with fiberglass to allow test personnel to walk on it during test operations and also so it could be easily repaired if damaged during handling or the wind-tunnel test. Photographs of the model MLP installed in the TDT, with the Ares I-X FTV model and balance, are presented in Fig. 46 and Fig. 47. 


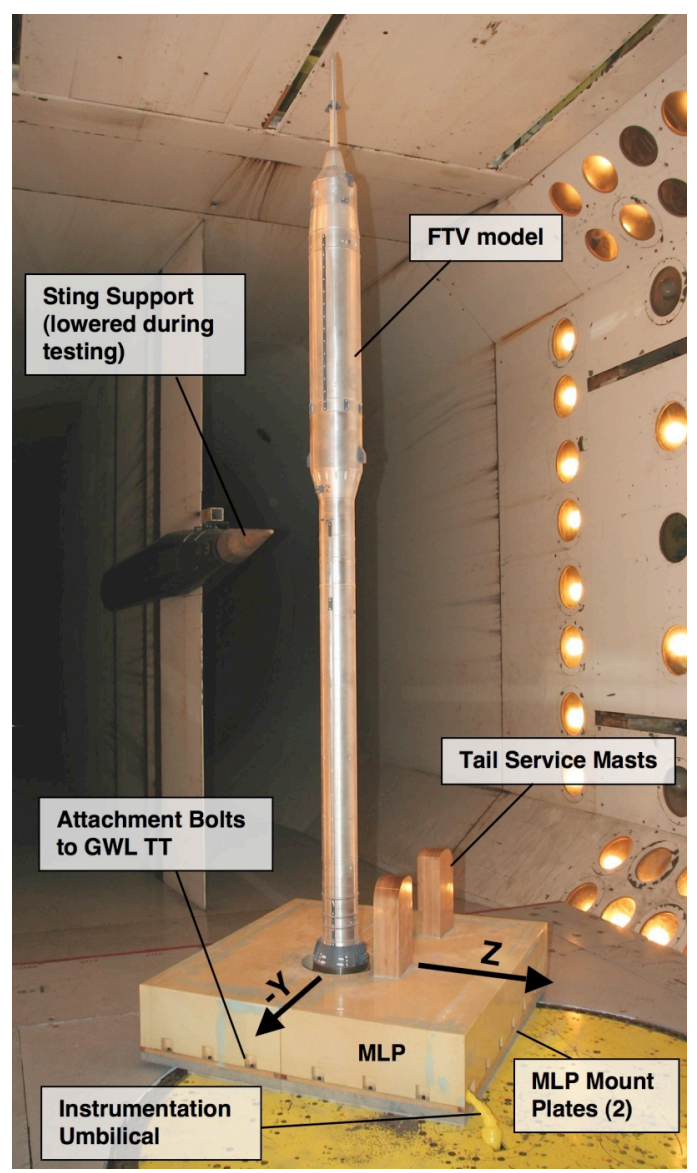

Figure 46. Ares I-X GWL model in TDT - MLP details. Rollout configuration - looking downstream.

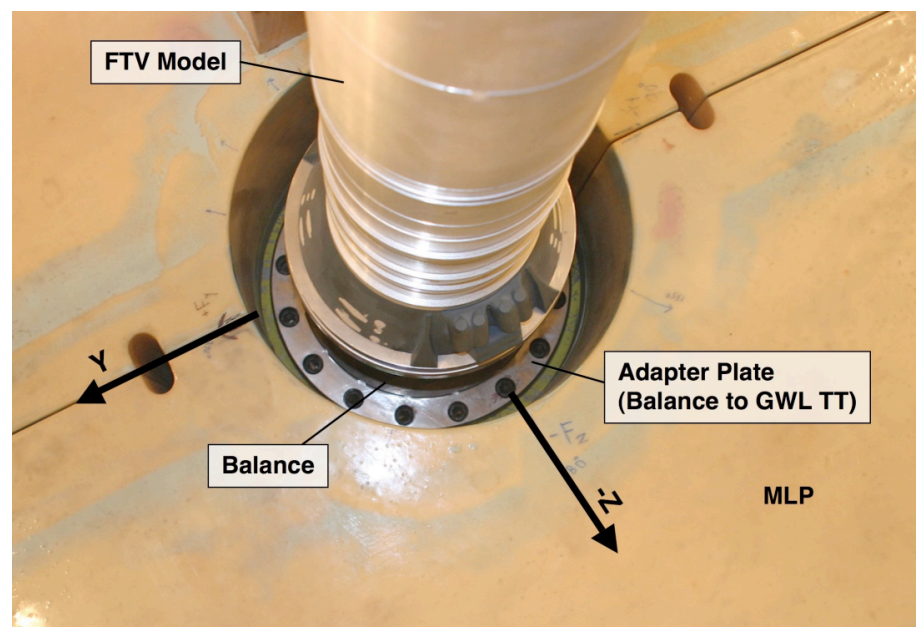

Figure 47. Ares I-X GWL model - MLP details. Model and balance location and orientations.

The model components that would simulate the primary KSC Launch Complex 39B structures, the FSS and RSS, were conceived to be simplified versions of the full-scale FSS and RSS. The model-scale FSS and RSS were designed to be relatively stiff and robust to withstand the predicted maximum test conditions. The profile and correct relative positions of the model FSS and RSS, with respect to the launch vehicle model and model MLP, created the basic aerodynamic blockage and interference effects on the Ares I-X FTV model similar to what would occur on the full-scale vehicle during on-pad stay and launch configurations. The model FSS and RSS were not in contact with the launch vehicle model, balance, or model MLP during the wind-tunnel test so any actual physical 
connections between the full-scale FSS and RSS and the Ares I-X FTV and MLP were not simulated. The model FSS and RSS were 0.0405-scale components attached to the GWL TT surface and positioned to allow the model assembly to rotate $360^{\circ}$ during testing in both the on-pad stay and launch (RSS rotated away from the Ares I-X FTV) configurations. The model FSS and RSS included major structural components of the full-scale structures and any attached structures that were considered critical for GWL testing. One such attached structure was the Vehicle Stabilization System (VSS), used with the Ares I-X FTV during on-pad stay to minimize vehicle motion due to ground winds at KSC LC-39B.

The final geometry and components of the model FSS and RSS for the Ares I-X GWL model were specified based upon CAD models. The model RSS was able to be positioned near the launch vehicle model for the on-pad stay configuration and then rotated away from the launch vehicle model for the launch configuration. The level of structural detail for the FSS and RSS that would be present in these wind-tunnel model components and all included structural elements were represented by model scale structure of similar shape and orientation to the most practical extent. The FSS had several large structures attached to it. The structures selected for inclusion on the model FSS during the test were the VSS, Upper Service Access Arm (USAA) (modified Shuttle Gaseous Oxygen Vent Arm), and the Shuttle Gaseous Oxygen Hood Maintenance Arm (USAA Platform for test) modified for the Ares I-X launch. The planned lightning tower was also designed and fabricated for the test but was not used during the test. The new KSC LC-39B Lightning Protection System for the Constellation Program was completed prior to rollout of the Ares I-X FTV and this eliminated the test requirement for a lighting tower on top of the model FSS. Photos of the full-scale FSS and RSS showed a great deal more structure and detail than was available in the CAD files. There was, however, no verified definition of this additional structure and there was a practical limit to the detail that could be included in the test components and the level of detail required to accomplish the test technical objectives. A view of the CAD file geometry for the KSC LC-39B, as planned for Ares I-X operations, is presented in Fig. 48.

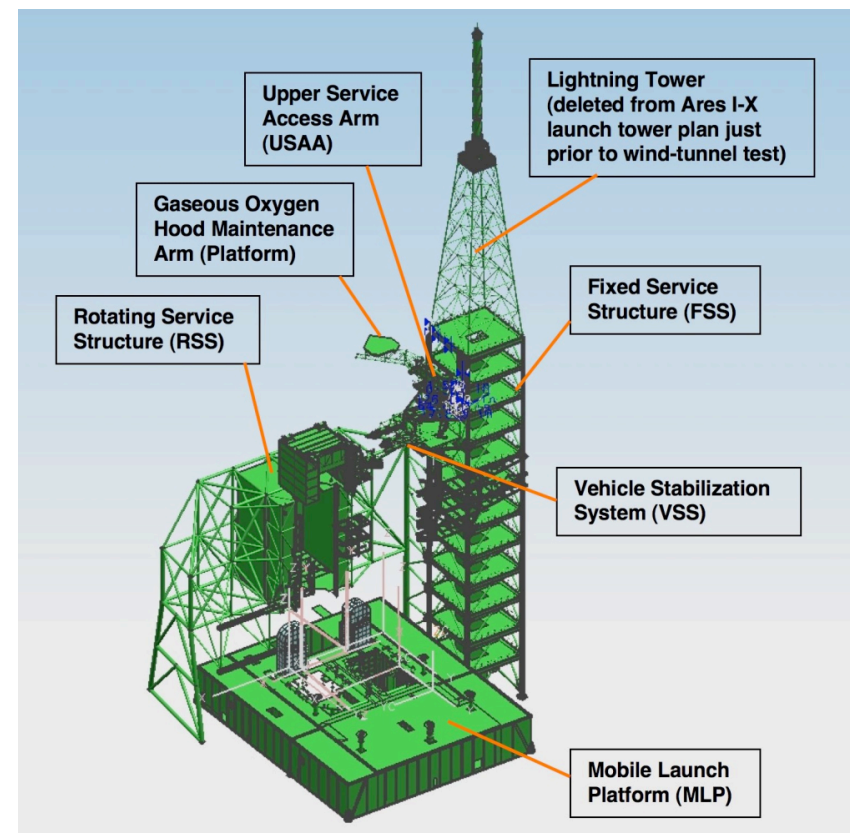

Figure 48. CAD model for KSC LC-39B structures for Ares I-X FTV, on-pad stay.

There were no specific requirements for mass and stiffness for the model FSS, RSS, and attached components although it was desired that these have minimal deflection and no significant dynamic motion during wind-tunnel testing. The model FSS, RSS, and attached components, such as the VSS, were required to withstand predicted loads in any direction for a planned maximum test dynamic pressure of $150 \mathrm{psf}$

The model FSS primary structure was comprised of four steel plates bolted and pinned together to form an approximately nine-foot long center beam that represented the elevator shaft of the full-scale FSS. The beam was bolted and pinned inside a collar that was part of a steel weldment that mounted the entire model FSS assembly to the surface of the GWL TT. The eleven model FSS floor sections were fabricated by welding together steel plates and tubing. These independently mounted floor sections were installed on the model FSS by sliding them down the length of the model FSS elevator shaft beam and securing them in place using angled steel clips. The floor sections 
were not connected to each other so that the center beam carried all aerodynamic loads on the model FSS. The hinge post assembly, to which the model RSS was attached and manually rotated about for on-pad stay or launch configurations, was connected to the model FSS through another welded steel plate and tubing assembly that reached through the floor sections and attached directly to the model FSS center beam. The part that simulated the model VSS was comprised of steel plates that had been welded together to replicate the primary full-scale VSS truss structure. The portion of the full-scale VSS that contained the springs and dampers and attach to the Ares I-X FTV was simulated using two pieces fabricated from polycarbonate material. These two pieces could be manually secured in the attached (did not actually contact model) or retracted position to simulate on-pad stay and launch configurations, respectively. The two remaining parts, the model USAA and USAA platform were also fabricated from welded steel plates and mounted to the model FSS using bolts and pins. The model USAA could also be manually positioned in the extended (on-pad stay) or retracted (launch) configuration. Details of the model FSS are presented in Fig. 49 for the Ares I-X GWL model in the on-pad stay configuration.

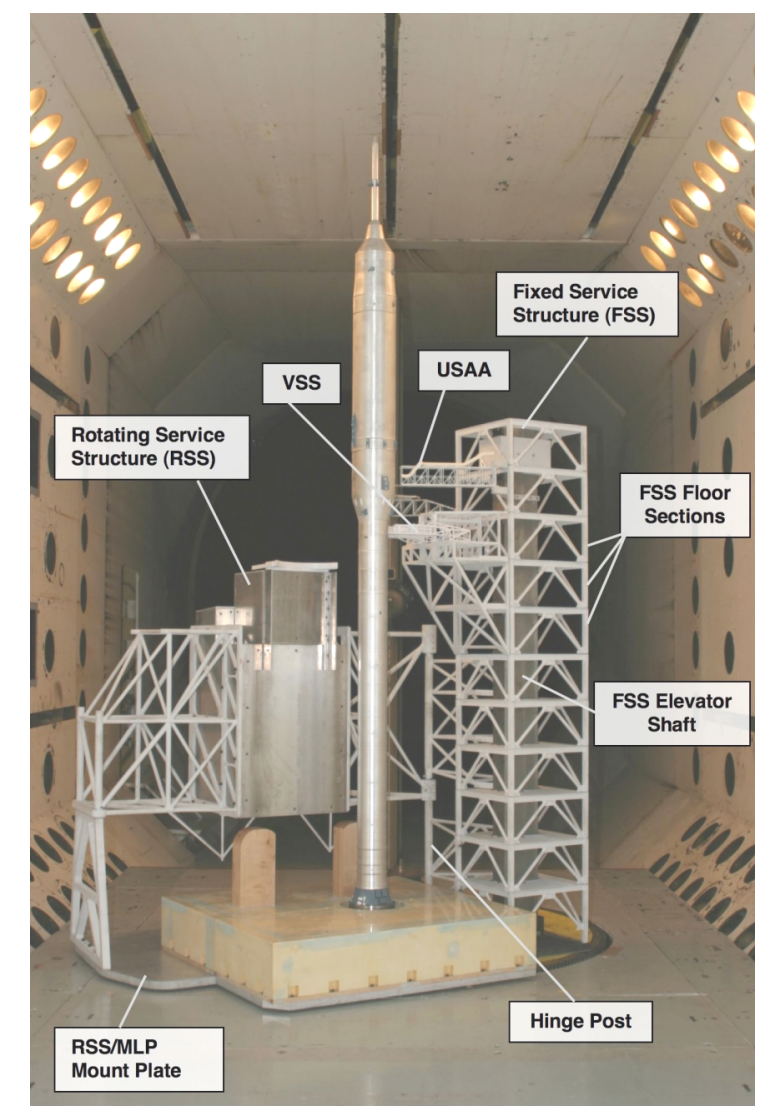

Figure 49. Ares I-X GWL model installed in TDT, looking downstream. On-pad stay configuration.

The model RSS was comprised of the large center box structure that simulated the Shuttle Payload Changeout Room and associated service areas, and the bridge and truss structure that supported it and attached it to the model FSS through the hinge post that was bolted to the GWL TT. The center box was fabricated from aluminum plate and honeycomb bolted and bonded together to form a rigid structural box. This center box was bolted to the supporting bridge and truss structure, constructed from welded steel plate and tubing assemblies, using bolts and pins. The model RSS was mounted to a large aluminum mount plate that was bolted and pinned to the surface of the GWL TT. The model RSS could be mounted in the closed or open (retracted) positions, for on-pad stay and launch configurations, respectively. In the open position, shown in Fig. 50, the model RSS was mounted to an independent mount plate. In the closed position, the model RSS and MLP shared a mount plate, as presented in Fig. 49. There was no instrumentation on the model FSS or RSS. 


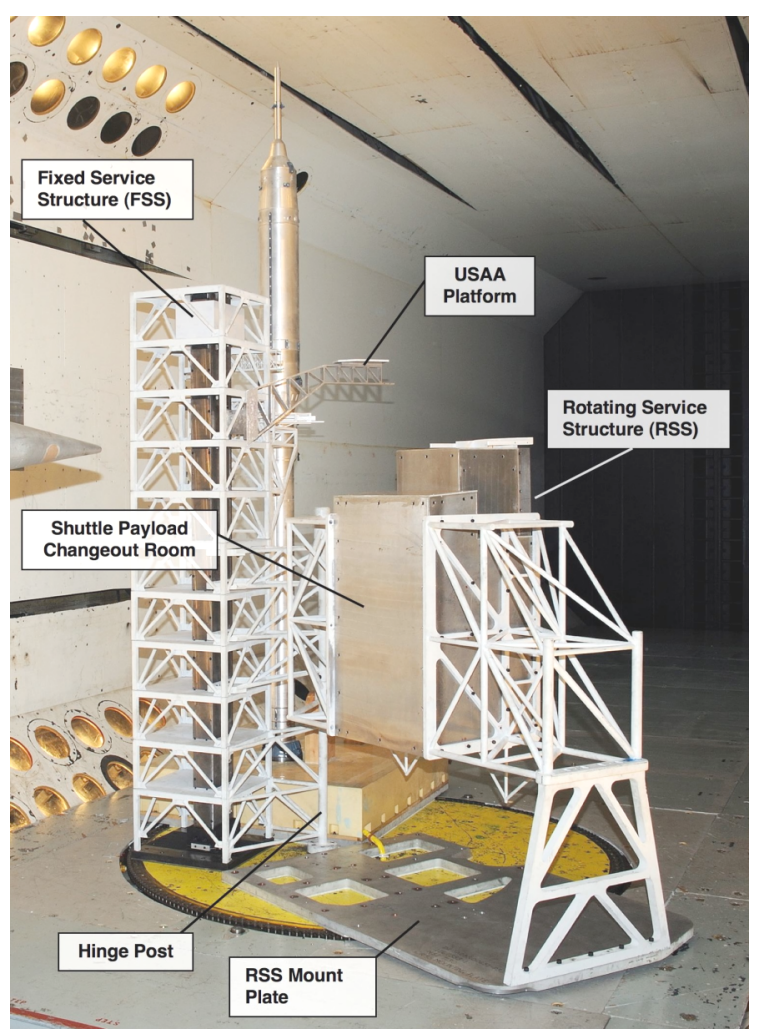

Figure 50. Ares I-X GWL model installed in TDT, looking downstream. On-pad launch configuration.

For completeness, the orientation of the primary LC-39B structures, FSS and RSS, simulated in the Ares I-X GWL model test in the TDT, is depicted in Fig. 51. The position of the RSS is shown for the on-pad stay and onpad launch configurations. 


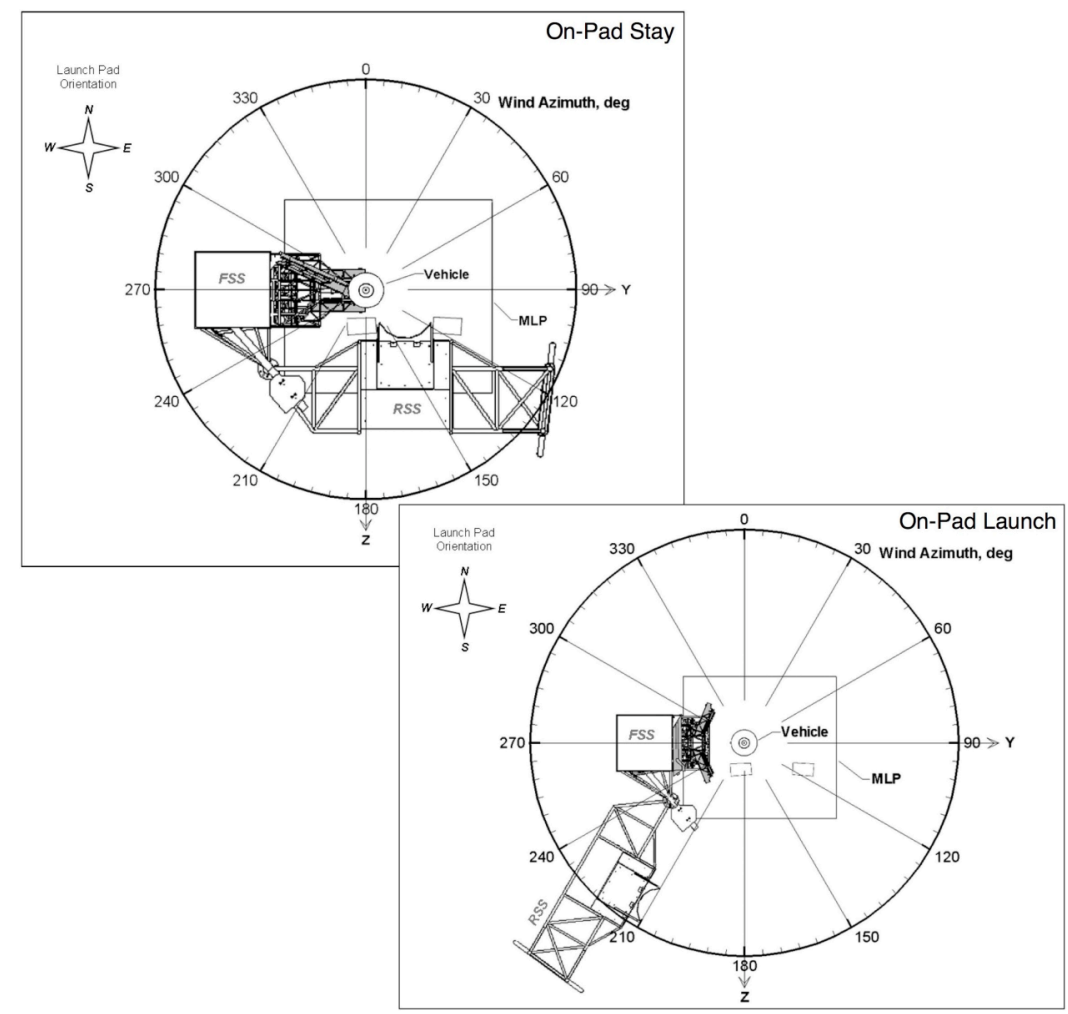

Figure 51. Orientation of FSS and RSS for on-pad stay and launch configurations.

\section{Configurations}

The first Ares I-X GWL model configuration tested, rollout, simulated rollout of the Ares I-X FTV on the MLP. All 8 ballast weights were installed. No damper was installed. As for all configurations tested, all protuberances were installed on the launch vehicle model and grit applied to the entire launch vehicle model surface, except for the LAS tower, to simulate full-scale Reynolds number flow conditions. The majority of test data was acquired by performing velocity sweeps, test section velocity typically increased from $50 \mathrm{ft} / \mathrm{s}$ to $200 \mathrm{ft} / \mathrm{s}$, where the turntable angle (flow azimuth) would be held constant, and data acquired at $5 \mathrm{ft} / \mathrm{s}$ increments in test section flow velocity. The velocity sweeps were conducted over a flow azimuth range of $0^{\circ}$ to $355^{\circ}$ in increments of $5^{\circ}$. Data acquired during the velocity sweeps was used to identify velocities and flow azimuths where significant Ares I-X FTV model dynamic response was present. Tunnel flow velocity and turntable angle was varied in smaller increments in these regions of interest and additional data acquired to properly define model peak response at these potentially critical conditions. The rollout configuration is shown in Fig. 52. 


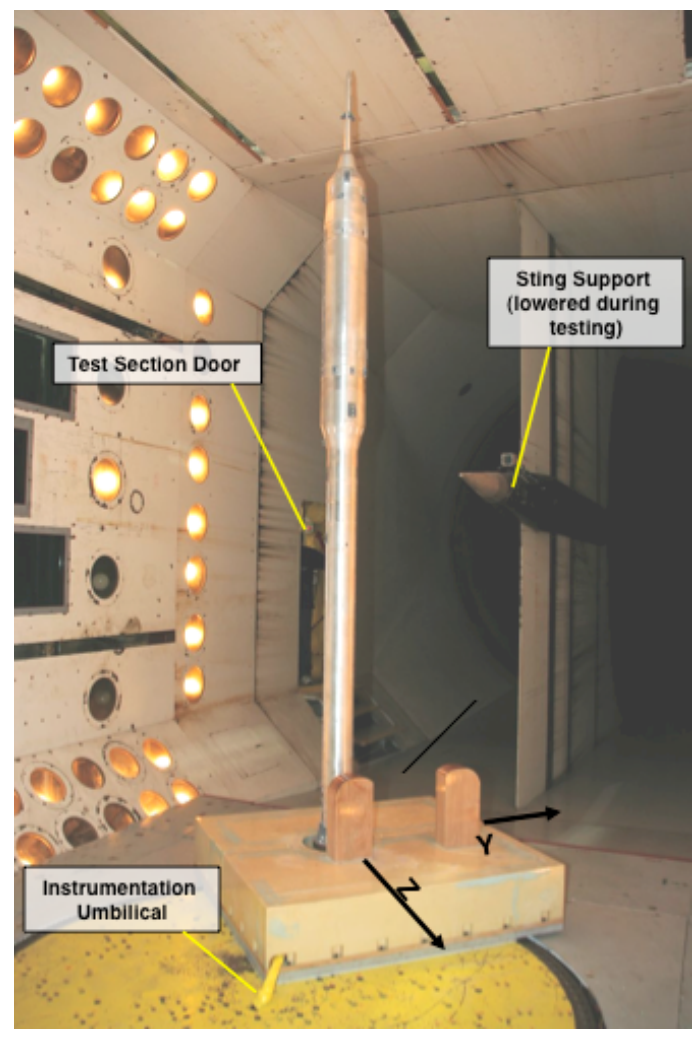

Figure 52. Ares I-X GWL model, rollout configuration - looking downstream.

The second model test configuration, on-pad stay, simulated the Ares I-X FTV at the KSC LC-39B. The model included the rollout configuration with the addition of the FSS, and the RSS in the closed, on-pad stay, position. The VSS and Upper Stage Access Arm (USAA) were positioned in the on-pad stay positions (extended) near the Ares I-X FTV model. The majority of test data was acquired by performing velocity sweeps. Test section velocity was typically increased from $50 \mathrm{ft} / \mathrm{s}$ to $180 \mathrm{or} 190 \mathrm{ft} / \mathrm{s}$, where the flow azimuth would be held constant, and data acquired at $5 \mathrm{ft} / \mathrm{s}$ or $10 \mathrm{ft} / \mathrm{s}$ (typical) increments in test section flow velocity. The velocity sweeps were conducted over a flow azimuth range of $0^{\circ}$ to $350^{\circ}$ in increments of $10^{\circ}$ (typical). Data acquired during the velocity sweeps was used to identify velocities and flow azimuths where high model response was present although the test matrix was less extensive than that for the rollout configuration. Tunnel flow velocity and flow azimuth were varied in smaller increments in these regions of interest and additional data acquired to properly define model peak response at these potentially critical conditions. The on-pad stay configuration is shown in Fig. 53. A close-up view of the VSS and USAA for the on-pad stay configuration is shown in Fig. 54. 


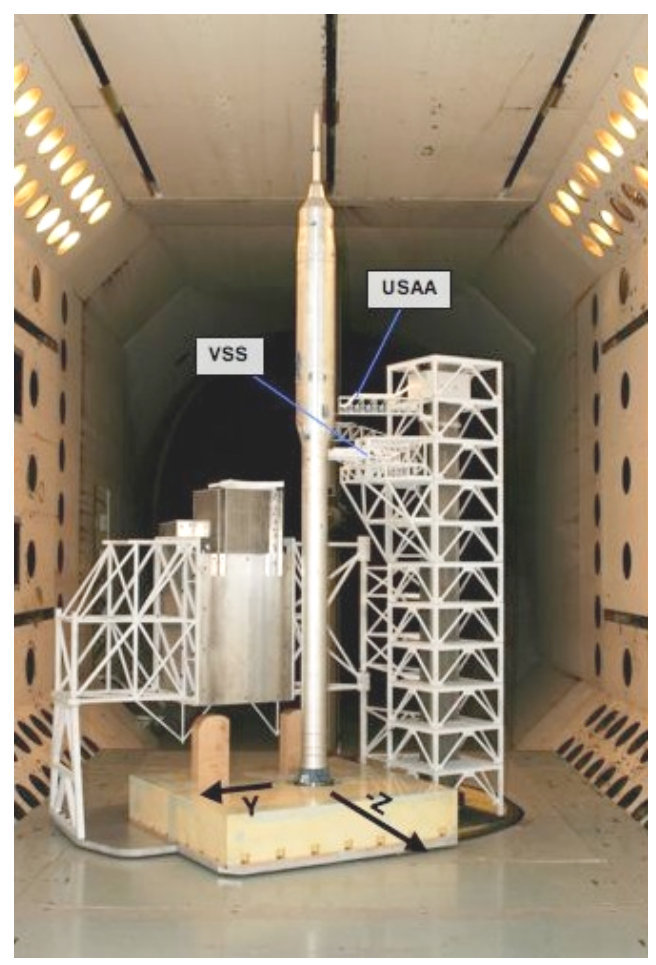

Figure 53. Ares I-X GWL model, on-pad stay configuration - looking downstream.

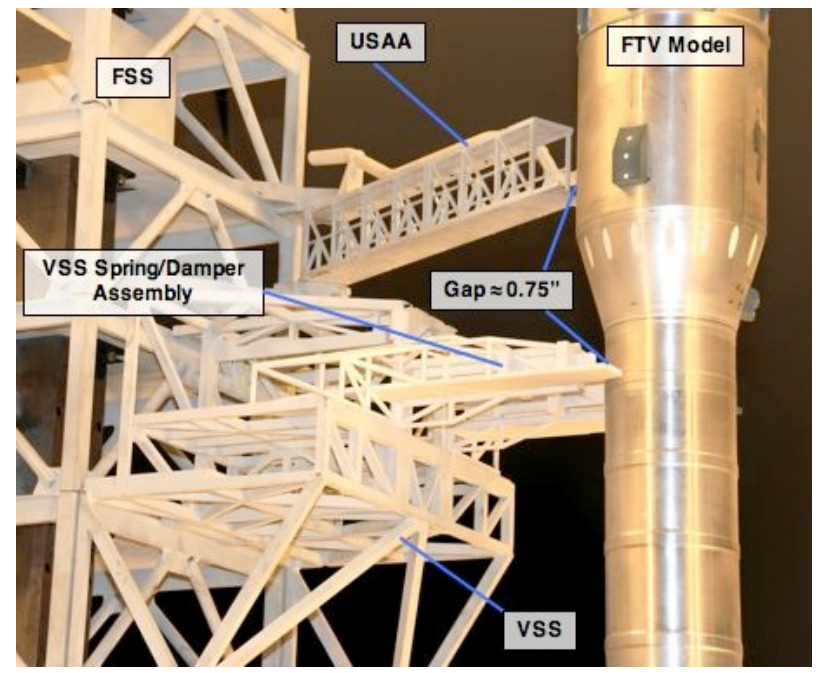

Figure 54. Close up view of VSS and USAA for on-pad stay configuration.

The third model configuration, on-pad launch, simulated the Ares I-X FTV at KSC LC-39B with the RSS open (retracted) in the launch position. The model components were the same as for the on-pad stay configuration but with the model VSS and USAA retracted, as the actual components were for launch of the Ares I-X FTV. The majority of test data was acquired by performing velocity sweeps with test section velocity typically increased from $45 \mathrm{ft} / \mathrm{s}$ to approximately $110 \mathrm{ft} / \mathrm{s}$, where the flow azimuth was held constant, and data acquired at $5 \mathrm{ft} / \mathrm{s} \mathrm{or} 10 \mathrm{ft} / \mathrm{s}$ (typical) increments in test section flow velocity. The lower maximum test section velocity represented the lower full-scale wind speed requirement for the Ares I-X FTV, immediately prior to launch. The velocity sweeps were conducted for turntable angles of $0^{\circ}$ to $355^{\circ}$ in increments of $5^{\circ}$ or $10^{\circ}$. Data acquired during the velocity sweeps was used to identify velocities and flow azimuths where high model response was present although, as with the onpad stay configuration, for a test matrix that was less extensive than that for the rollout configuration. Tunnel flow velocity and flow azimuth were varied in smaller increments in regions of interest and additional data acquired to 
properly define Ares I-X FTV model peak response at these potentially critical conditions. The on-pad launch configuration is presented in Fig. 55.

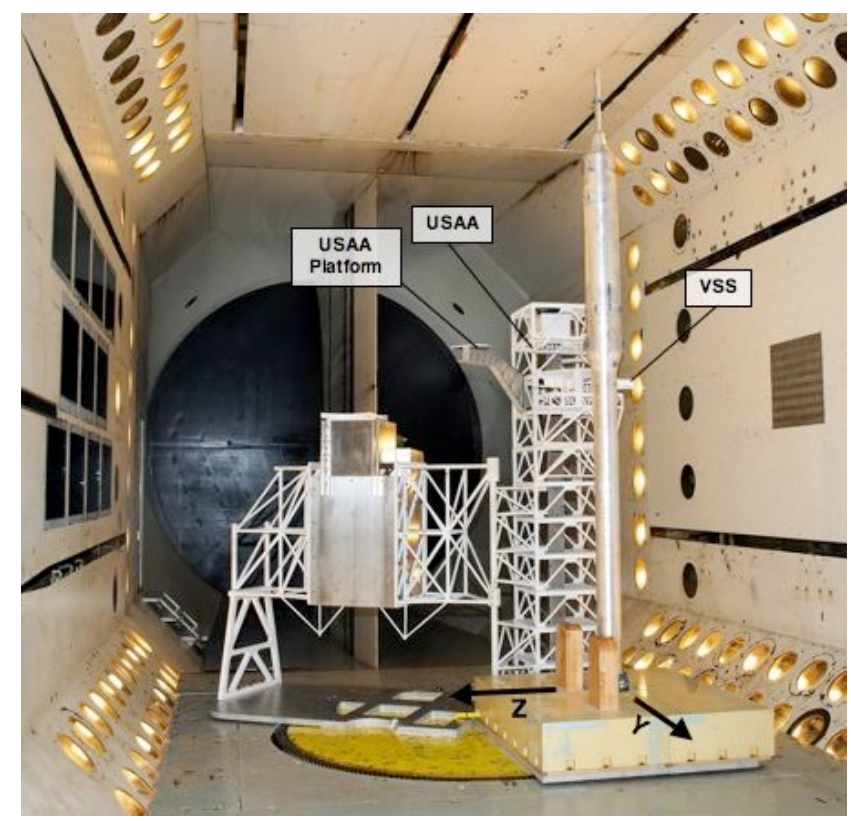

Figure 55. Ares I-X GWL model, on-pad launch configuration - looking downstream.

The fourth and last Ares I-X GWL model test configuration, rollout with damper installed, simulated rollout of the Ares I-X FTV on the MLP with increased structural damping for the $1^{\text {st }}$ bending mode in the Z-direction (1Z) and $2^{\text {nd }}$ bending mode in the $\mathrm{Y}$-direction $(2 \mathrm{Y})$. These modes were selected for increased damping based on test data acquired on the rollout configuration. Ballast weight \#1 was removed and replaced with the internal damper specifically tuned to increase damping of modes $1 \mathrm{Z}$ and $2 \mathrm{Y}$ to a value of approximately $1 \% \mathrm{C} / \mathrm{Cc}$. Actual values of damping for both modes were considerably higher and impact hammer damping tests conducted at the beginning and end of testing of this configuration indicated that the effectiveness of the damper increased during use. The damper had little effect on the frequency of the four critical modes, as expected, because the weight of the damper with tuning weights installed was approximately the same as ballast weight \#1. Average values for frequency and damping for the four modes are summarized in Table 11. The test matrix used for this configuration was significantly smaller than those for the previous configurations due to a focus on known regions of high response and a limit on available test time. The majority of test data was acquired by performing velocity sweeps over narrow ranges of test section velocity at specific flow azimuths. A few turntable sweeps over narrow ranges in flow azimuth were also conducted where the test section velocity remained constant. Significant reductions in model response were observed in comparison to that encountered for the original rollout configuration where only inherent Ares I-X FTV model structural damping was present.

Table 11. Average frequency and damping of critical modes at start and end (red and underlined) of wind-on testing for Ares I-X GWL model in TDT, rollout configuration with damper installed.

\begin{tabular}{|c|c|c|c|}
\hline Mode & Description & $\begin{array}{c}\text { Average Frequency, } \\
\text { Hz. }\end{array}$ & $\begin{array}{c}\text { Average Damping, } \\
\text { (\% C/Cc) }\end{array}$ \\
\hline 1 & 1st Bending, Y-axis (1Y) & $6.70 / \underline{6.70}$ & $0.22 / \underline{\underline{0.28}}$ \\
\hline 2 & 1st Bending, Z-axis (1Z) & $8.26 / \underline{8.26}$ & $1.31 / \underline{1.83}$ \\
\hline 3 & 2nd Bending, Y-axis (2Y) & $35.72 / \underline{35.66}$ & $1.17 / \underline{1.48}$ \\
\hline 4 & 2nd Bending, Z-axis (2Z) & $43.42 / \underline{43.44}$ & $1.03 / \underline{0.99}$ \\
\hline
\end{tabular}




\section{Test Implementation and Conduct}

\section{A. Tunnel Flow Conditions}

Test section flow conditions were determined by test medium, tunnel total pressure (generally near $2000 \mathrm{psf}$ ), and tunnel motor rpm. Test medium was chosen prior to wind-on operations and was R-134a for most wind-on testing test. Tunnel total pressure was generally set prior to wind-on conditions although it was varied during windon operations if it was more efficient to do so than when the tunnel was off-line. Test-section velocity was generally controlled by varying tunnel motor rpm. Velocity sweeps were conducted by increasing tunnel $\mathrm{rpm}$ to, and acquiring data at, each specified velocity for a set turntable angle (flow azimuth). These sweeps were performed to identify velocities of interest where vortex shedding appeared to occur as indicated by significantly increased model response. In addition, turntable angle sweeps were sometimes conducted to identify flow azimuths of interest for a specified velocity.

\section{B. GWL Turntable Operation}

During the majority of wind-on testing, suction (vacuum) was supplied to the GWL TT to ensure the upper plate was restrained to the base plate to the maximum extent possible. The applied suction resulted in as near to a cantilevered base boundary condition as practical for the model. Acquisition of data only occurred when adequate suction was applied to the GWL TT as indicated by a digital pressure gauge on the GWL TT controller. Rotation of the model was accomplished by switching from vacuum to a positive supply pressure (air or heavy gas) that lifted the turntable upper plate at least 0.020 " up from the base plate.

\section{Data Acquisition, Reduction, and Analysis}

Data was acquired and processed using the TDT Data Acquisition System (DAS). Data was acquired at a rate of 500 frames (samples per data channel) per second, generally for a period of 15 seconds. All dynamic data was antialias filtered at $200 \mathrm{~Hz}$. All data and computed quantities such as balance measured moments and forces, accelerations, unsteady pressures, calculated forces and moments resolved in the wind axis system, tunnel parameters, and equivalent full-scale quantities were time correlated. Data was continually processed and reviewed during the test to check for repeatability and quality, as practical, and to determine the next test conditions, during the testing of each model configuration.

The ESP system scanned data at a rate of 10 samples per second and only the mean values of the data from the ESP system were considered accurate.

Converting model-scale wind tunnel data into equivalent full-scale values was accomplished by reducing the wind tunnel data into a nondimensional coefficient form using model-scale reference values. The equivalent fullscale quantity was then calculated by converting the coefficient back into dimensional form using full-scale reference values.

The response of the first bending mode was the only mode of interests for the GWL CM since second mode response was not an objective and little, if any, second mode response was observed during that test. As stated previously, however, first and second mode response, with particular interest in second bending mode response, were encountered during the Ares I-X GWL model test. During wind tunnel testing of the Ares I-X GWL model, bending moments were only measured at the balance moment center and not at other areas of the vehicle. The need to quantitatively isolate the loading associated with first modes and second modes was identified. This was accomplished through the use of a digital elliptical band-pass filter (not used during GWL CM test and data reduction/analysis).

Fig. 56 contains a power spectral density (PSD) plot of the base bending moment due to lift and the base bending moment due to drag, LBM and DBM respectively, of a typical data record from the Ares I-X GWL model test. The data shown in Fig. 56 is unfiltered and peak responses at the frequencies of vibration modes $1 \mathrm{Y}, 1 \mathrm{Z}, 2 \mathrm{Y}$, and $2 \mathrm{Z}$ are depicted in addition to the bandwidths of the filters used to acquire filtered data. 


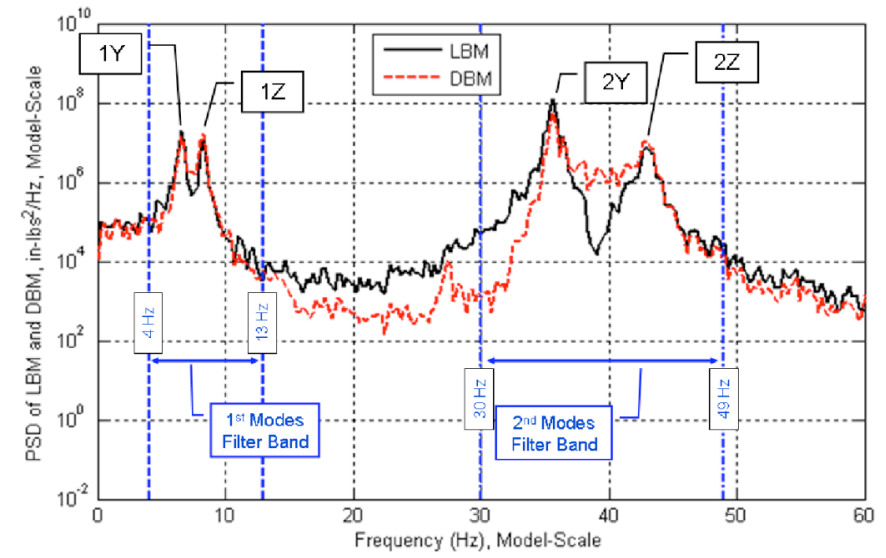

Figure 56. PSD of bending moments with filter bands illustrated, model scale, Ares I-X GWL model.

LBM and DBM are quantities of base bending moment that are resolved in the wind-axis system. Therefore, an LBM or DBM time history will contain vibration contributions of the bending modes about both the $\mathrm{Y}$ and $\mathrm{Z}$ model axes as demonstrated here. Analyzing the base bending moments in the model-axis system, $\mathrm{M}_{\mathrm{Y}}$ and $\mathrm{M}_{\mathrm{Z}}$, isolated the vibrations of the modes in the $\mathrm{Y}$ and $\mathrm{Z}$ axes. This method allowed better analysis of individual contributions of modes $1 \mathrm{Y}, 1 \mathrm{Z}, 2 \mathrm{Y}$ and $2 \mathrm{Z}$; however, the greater interest for the current study was to analyze the dynamics of bending moment due to lift. Therefore, the filter is demonstrated on the quantity LBM.

Fig. 57 contains the unfiltered time-history data of the quantity LBM for a one-second duration of a typical data record. In addition to the unfiltered time-history data for LBM, Fig. 57 also contains the results of band-pass filtered data for the first and second bending modes.

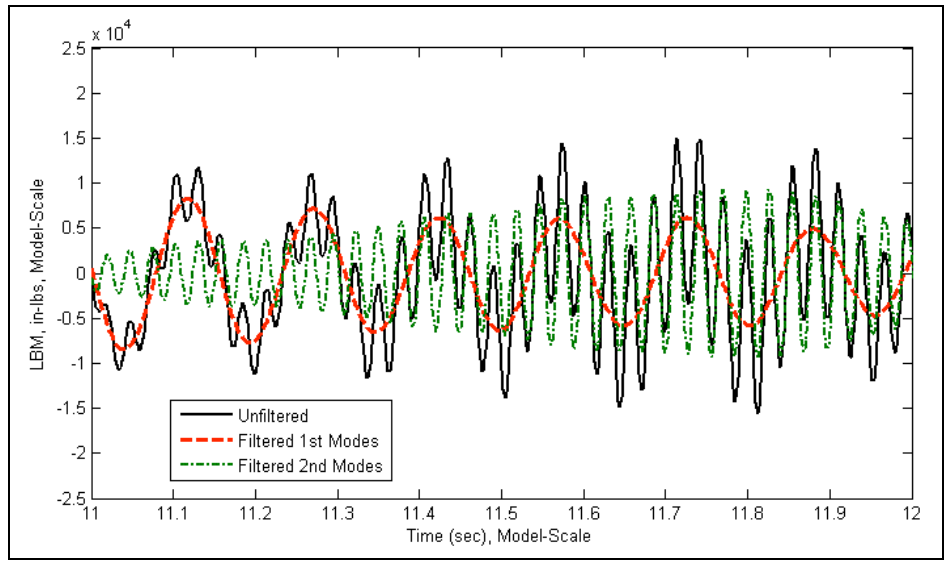

Figure 57. Tine history of filtered and unfiltered data for LBM, model scale, Ares I-X GWL model.

Dynamic motion of any real structure can be represented as a linear combination of an infinite number of individual modal vibrations. If all dynamic motion of the wind tunnel model can be attributed to only the modes $1 \mathrm{Y}, 1 \mathrm{Z}, 2 \mathrm{Y}$ and $2 \mathrm{Z}$, then a linear addition of the filtered modes displayed in Fig. 57 would equal the original unfiltered signal. Fig. 58 contains a time history of the original unfiltered LBM data and a linear sum of the filtered components for a one-second duration of a typical data record showing extremely close agreement and verifying this assumption. 


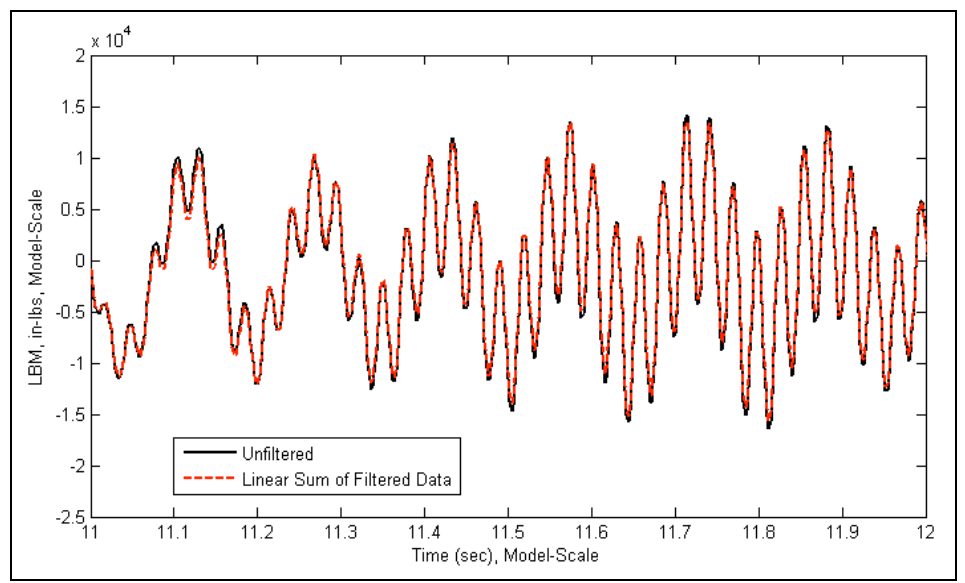

Figure 58. Comparison of unfiltered data and linear sum of filtered data for LBM, model scale, Ares I-X GWL model.

\section{Data Quality Assurance}

Data quality is an extremely important aspect of any wind-tunnel test and additional requirements and challenges exist for the acquisition, reduction, and analysis of dynamic data. Extensive efforts were made during model fabrication, instrumentation selection and installation, and the set-up and conduct of the wind-tunnel test to provide confidence in the quality of the acquired data. All pressure instrumentation and accelerometers were calibrated and functionally checked before installation in the model. Pressure instrumentation was calibrated and functionally checked prior to wind-on testing and the calibrations were checked on a regular basis throughout the test. Base bending moment strain gauge bridges and the balance, for the GWL CM and Ares I-X GWL model, respectively, were calibrated in place in the TDT, with the models installed, and check loaded several times during the test. Unfortunately, accuracy of the force measurements for the Ares I-X GWL model balance was determined to be of too poor of quality to be acceptable. Phase and amplification checks were performed on the TDT DAS just prior to wind-on testing.

\section{Results and Discussion}

\section{A. Ground Wind Loads Checkout Model}

\section{Test Summary}

Checkout runs were conducted using Configuration \#1 with wind-on testing being comprised of two velocity sweeps in air at flow azimuths of 0 and 90 degrees. No additional wind-on testing was performed for Configuration \#1. Protuberances were installed on the model. Configuration \#2 was tested extensively in air at near atmospheric pressure and using heavy gas, R-134a, at total pressures of approximately 1000, 1500, and 2000 psf to determine Reynolds number effects on GWL characteristics, steady (drag) and dynamic (WIO) at various flow azimuths and velocities. Wind-on air testing for Configuration \#2 consisted almost entirely of flow azimuth sweeps at constant velocities of 40,60, 70, 80, 100, 109, and $250 \mathrm{fps}$ (model scale) with one velocity sweep of 60 to $305 \mathrm{fps}$ at a flow azimuth of $125^{\circ}$. Wind-on heavy gas testing for this configuration also consisted primarily of flow azimuth sweeps at specific test section velocities over a range of 50 to $220 \mathrm{fps}$ with data acquired at total pressures of approximately 1000,1500 , and 2000 psf. Several velocity sweeps were also conducted at and near flow azimuths where high first bending mode response (WIO) was encountered, such as $115^{\circ}$ and $310^{\circ}$. Grit was applied to the upper stage of the model and Configuration \#3 tested primarily at flow azimuths and velocities where high response had been encountered for Configuration \#2. Configuration \#3 was wind-on tested in air and heavy gas over a range of Reynolds numbers to investigate effects of the grit on drag and dynamic response and whether grit could be used to raise effective Reynolds number in preparation for testing of the Ares I-X GWL model. The protuberances were removed and the final configuration, Configuration \#4, was tested at very limited conditions in air and heavy gas. Velocity sweeps in air and heavy gas were performed at selected flow azimuths, primarily $310^{\circ}$, based on earlier test results, and flow azimuth sweeps conducted in heavy gas only for test section velocities of 100 and 115 fps. A brief summary of the test for each configuration is presented in Table 12. It should be noted that the convention for turntable angle (THETATT), illustrated in Fig. 59, was such that a turntable angle of 90 degrees equated to the fullscale vehicle on the pad experiencing wind from the East - 90 degrees (wind azimuth). 
Table 12. GWL Checkout Model, test run summary, model scale.

\begin{tabular}{|c|c|c|c|c|c|c|}
\hline Configuration \# & Data Runs, \# & Protuberances & US Grit & Medium, Air & Medium, R-134a & Re, /ft x 10 \\
\hline 1 & Checkout only & Off & No & $\mathrm{H} \approx 2000 \mathrm{psf}$ & None & $0.30-1.76$ \\
\hline 2 & 33 & On & No & $\mathrm{H} \approx 2000 \mathrm{psf}$ & $\mathrm{H} \approx 1000,1500,2000 \mathrm{psf}$ & $0.22-3.20$ \\
\hline 3 & 15 & On & Yes & $\mathrm{H} \approx 2000 \mathrm{psf}$ & $\mathrm{H} \approx 1000,1500,2000 \mathrm{psf}$ & $0.34-3.29$ \\
\hline 4 & 8 & Off & Yes & $\mathrm{H} \approx 2000 \mathrm{psf}$ & $\mathrm{H} \approx 1500,2000 \mathrm{psf}$ & $0.34-5.43$ \\
\hline
\end{tabular}

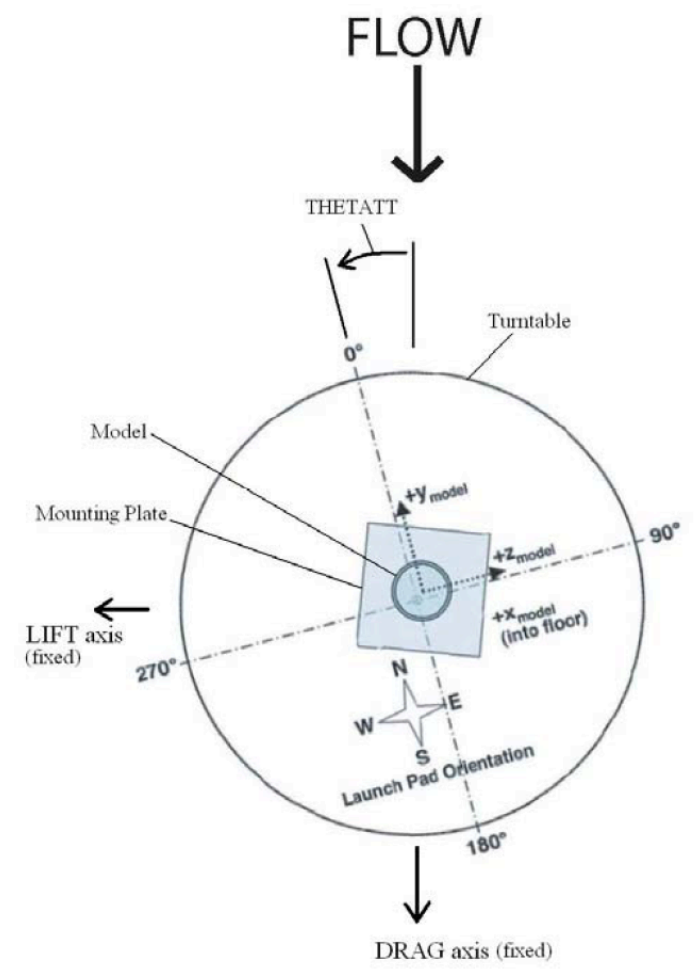

Figure 59. Top view illustration of GWL CM mounted on the GWL TT,

\section{Modal Testing and Analysis}

Model frequency and damping characteristics were monitored throughout the test. Model frequency data was acquired for each test configuration for correlation to analytical models, and to ensure that unexpected changes to model dynamic characteristics did not occur. Model damping data was acquired to establish percent-critical damping values for the model first four primary modes of vibration, first and second bending in the $\mathrm{Y}$ and $\mathrm{Z}$ directions. The data was also important to determine if model configuration changes and turntable angle had significant effects on model damping characteristics during testing as this could significantly impact model response due to vortex shedding.

Model response data acquired during the test also indicated that model frequency and damping characteristics might be related to turntable angle (flow azimuth). As a result of this and the requirement to properly characterize model modal characteristics, extensive wind-off model response data was acquired to determine how natural frequency and structural damping varied with turntable angle.

Model frequency and damping data was acquired using a large impact test hammer with a hard rubber tip. The hammer was used to excite the critical modes of vibration by striking the model sharply near the interstage region at intervals of 4-5 seconds while time history data was acquired for all model instrumentation. Analysis of the data indicated that model damping characteristics were affected by turntable angle.

Wind-off hammer-rap model response data for the first bending mode(s) are presented in Fig. 60 and Fig. 61. Ground vibration testing and hammer raps clearly indicated the existence of two orthogonal modes of vibration for the first (1st bending) and second (2nd bending) natural vibration modes of the model. This was likely the result of small asymmetries in model stiffness caused by the base mounting plate and primary protuberances. Analysis of 
hammer-rap results indicated that the frequency of the second mode did vary slightly with turntable angle and that structural damping of the first two model vibration modes (1st and 2 nd bending) varied with turntable angle. The first bending mode frequencies were approximately $16.1 \mathrm{~Hz}$ and $16.6 \mathrm{~Hz}$ in the model $\mathrm{Y}$-axis and Z-axis, respectively. Second bending mode frequencies were approximately $65.8 \mathrm{~Hz}$ (Y-axis) and $67.4 \mathrm{~Hz}$ (Z-axis).

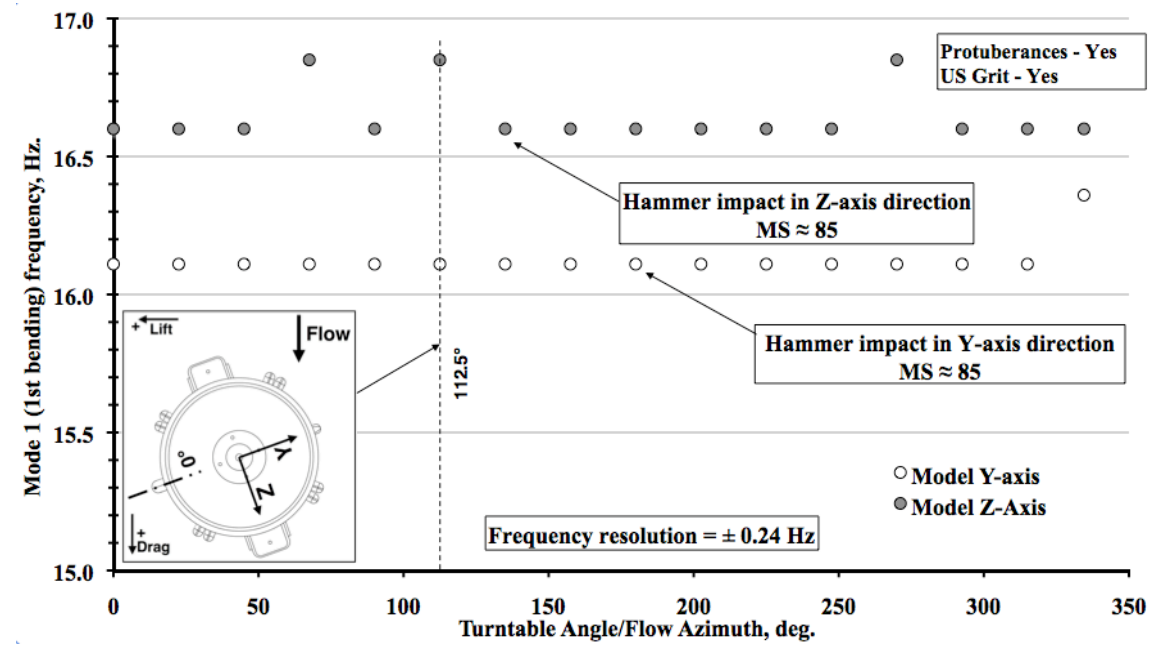

Figure 60. Effect of GWL TT angle on 1st bending mode frequency. GWL CM, Configuration \#3. Wind-off hammer raps.

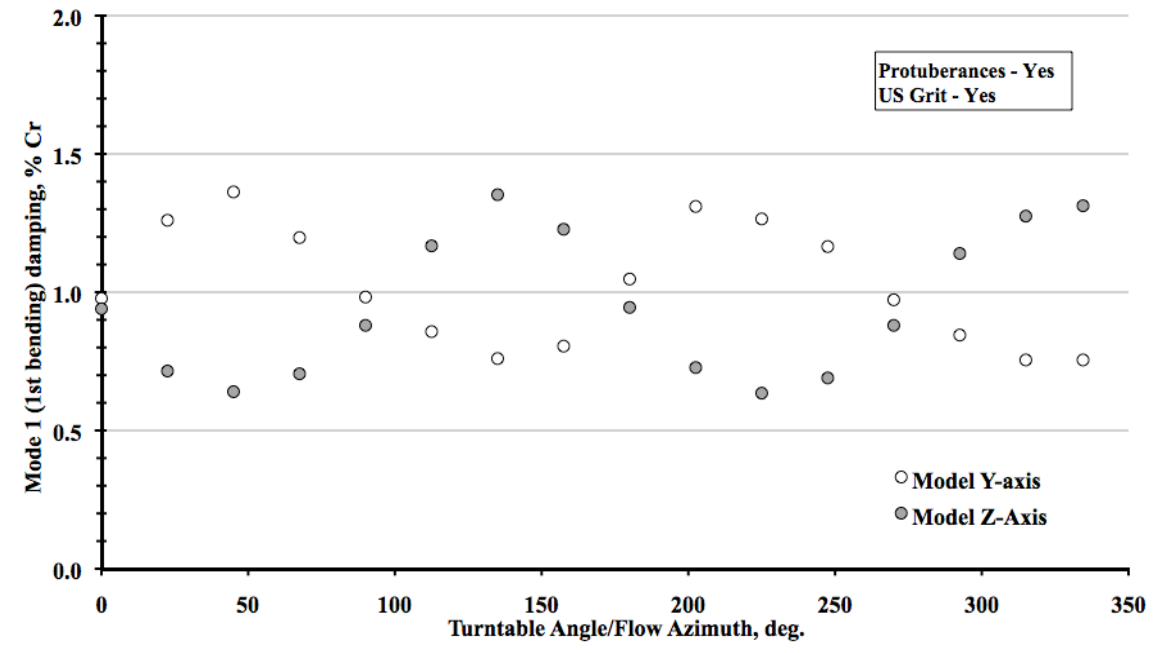

Figure 61. Effect of GWL TT angle on 1st bending mode damping, \%Cr. GWL CM, Configuration \#3. Wind-off hammer raps.

\section{3. $\quad$ Test Results}

The primary results from the GWL CM wind-tunnel test were the steady and dynamic base bending moment data since base bending moment data was important in determining critical loads at the base of the Ares vehicle and for decisions regarding the design and test of the then planned Ares I-X GWL model. The response (accelerometer) and pressure data were considered useful for better understanding the base bending moment data and model response characteristics and quantifying the aerodynamic forces acting on the GWL CM model.

The outputs of the base-bending moment strain-gauge bridges at model azimuth $0^{\circ}$ and $90^{\circ}$ were acquired and resolved during data acquisition to provide steady and dynamic base bending moment data and associated steady and dynamic coefficients in the lift and drag directions, $\mathrm{C}_{\mathrm{ML}}$ and $\mathrm{C}_{\mathrm{MD}}$, respectively. The same method was applied to output from the base-bending gauges at model azimuth $45^{\circ}$ and $135^{\circ}$. This data was used as a check and back-up to the $0^{\circ}$ and $90^{\circ}$ base bending moment gauges. Bending moment coefficients were calculated using the following formula: 


$$
C_{M}=[\text { Base bending moment, in-lbs }] /\left[q(\mathrm{psi}) \times A\left(\mathrm{in}^{2}\right) \times L(\text { in })\right]
$$

where

$$
A=\text { Model Projected Side Area }=1260.7 \text { in }^{2}
$$

and

$$
L=\text { distance from model base to centroid of model projected side area }=75.2 \mathrm{in} .
$$

These coefficients were also the force coefficients since, for the purpose of the above calculation, the center of force on the model was assumed to be at its centroid and the moment measured at the base of the model.

The primary configuration for the GWL CM test was Configuration \#2 since it was the most representative of the Ares I-X and Ares I CLV launch vehicles. As such, the dataset for that configuration was the most extensive and is the subject of the following data presentation and discussion. A brief discussion of the other configurations and results is presented at the end of this section. All data presented below was acquired with heavy gas (R-134a) as the test medium.

The two primary data values from the GWL CM test were the steady base bending moment coefficient in the drag direction, $\mathrm{C}_{\mathrm{MD}}$, and the dynamic base bending moment coefficient in the lift direction, $\mathrm{C}_{\mathrm{ML}}(\sigma)$. The windtunnel data for $\mathrm{C}_{\mathrm{MD}}$ indicated a wide variation in values for bending moment, and therefore the force coefficient, in the drag direction at low values for Reynolds numbers where the flow was still subcritical or transcritical. Once the flow, over most of the vehicle model, became supercritical, or fully turbulent, as shown in Fig. 4, the values for $\mathrm{C}_{\mathrm{MD}}$ converged into a narrow range of value of approximately 0.60 to 0.80 . The variation of $\mathrm{C}_{\mathrm{MD}}$ with test section velocity is shown in Fig. 62 for Configuration \#2 with heavy gas as the test medium. Variations within that range, indicated by the blue triangles in Fig. 62, were primarily due to the orientation of significant protuberances (systems tunnels and LOX feedline) with respect to flow azimuth.

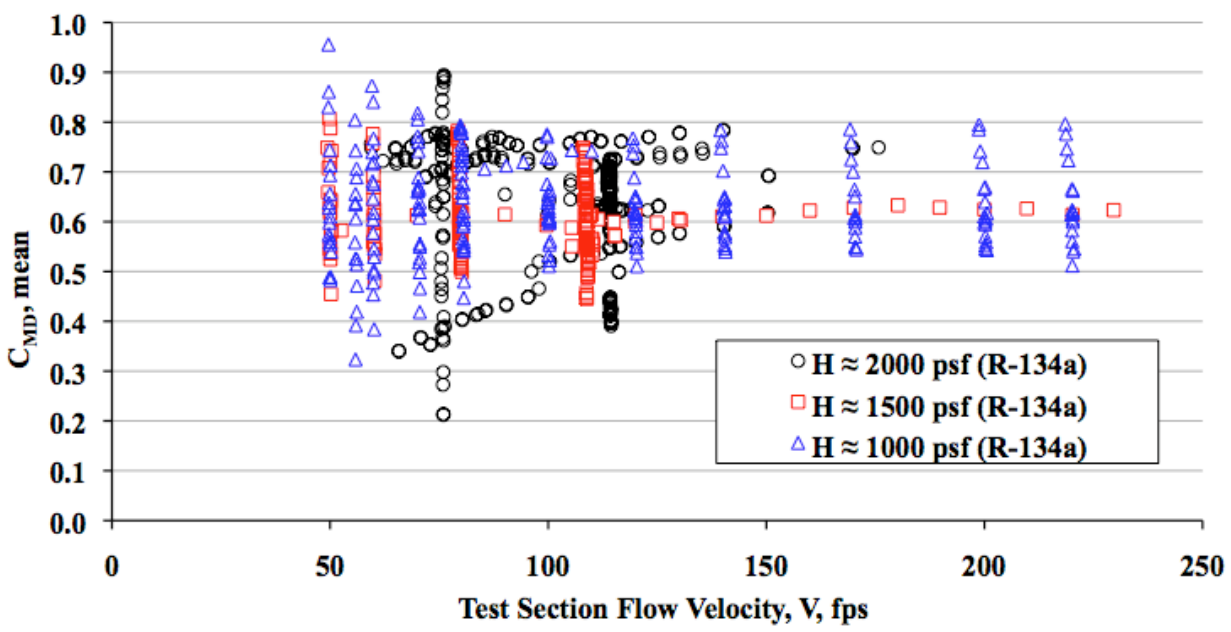

Figure 62. GWL Checkout Model. Base bending moment (mean) coefficient - drag direction. Configuration $\# 2$.

An example of how $\mathrm{C}_{\mathrm{MD}}$ varied with flow azimuth and as a function of Reynolds number (test section velocity) is shown in Fig. 63. The shape of the curve presented in Fig. 69 for the higher Reynolds number conditions was typical of that observed for higher Reynolds number testing for the GWL CM and later for the Ares I-X GWL model. The test section velocities of 75 and $114 \mathrm{fps}$ correspond to approximately 10 and 15 knots, full-scale, respectively. Based on these results, a drag coefficient of 0.80 was chosen for use in determining design loads for the Ares I-X GWL model. 


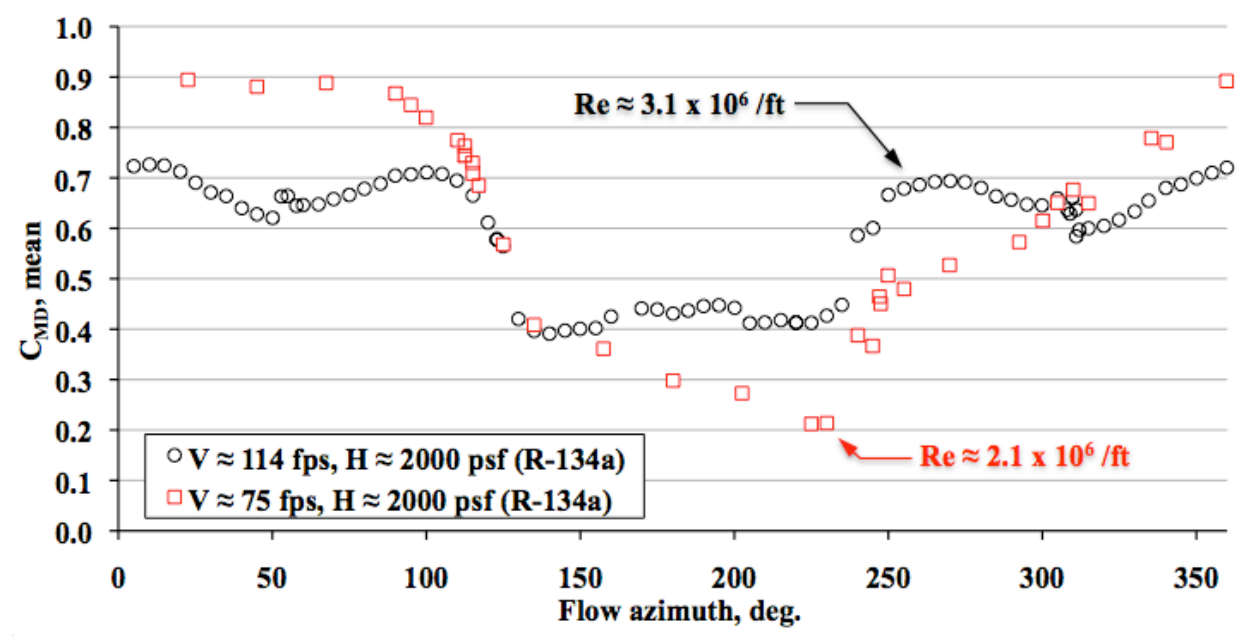

Figure 63. GWL Checkout Model. Effect of flow azimuth on base bending moment (mean) coefficient - drag direction. Velocity $=\mathbf{7 5}$ fps and 114 fps. Configuration \#2.

During the GWL CM test, two test section velocities were identified that resulted in high sinusoidal response, WIO, of the model although lesser regions of response were observed during the test. These two regions of strong WIO were the result of periodic vortex shedding, as evidenced by large amplitude sinusoidal response of the model, at a frequency at or near approximately $16 \mathrm{~Hz}$, the first bending mode frequency for the model. Wind tunnel data also indicated, as predicted, that the vortex shedding was more organized and periodic at higher Reynolds numbers (higher tunnel total pressure, H) and resulted in significantly higher model peak response for the observed WIO. These two peak responses also were found to only occur over a very narrow range of flow azimuth indicating that they were at least in part due to the protuberances ${ }^{9}$ acting to fix the separation point along certain lengths of the vehicle model. This fixed separation point (line) likely helped to synchronize and correlate the shed vortices along constant diameter sections of the model. The peak responses fell off more gradually as test section velocity was increased or decreased from peak response conditions and Strouhal frequency increased or decreased from a value of approximately $16 \mathrm{~Hz}$. Summary plots of dynamic bending coefficient in the lift direction as a function of test section velocity and flow azimuth for Configuration \#2 are shown in Fig. 64 and Fig. 65, respectively.

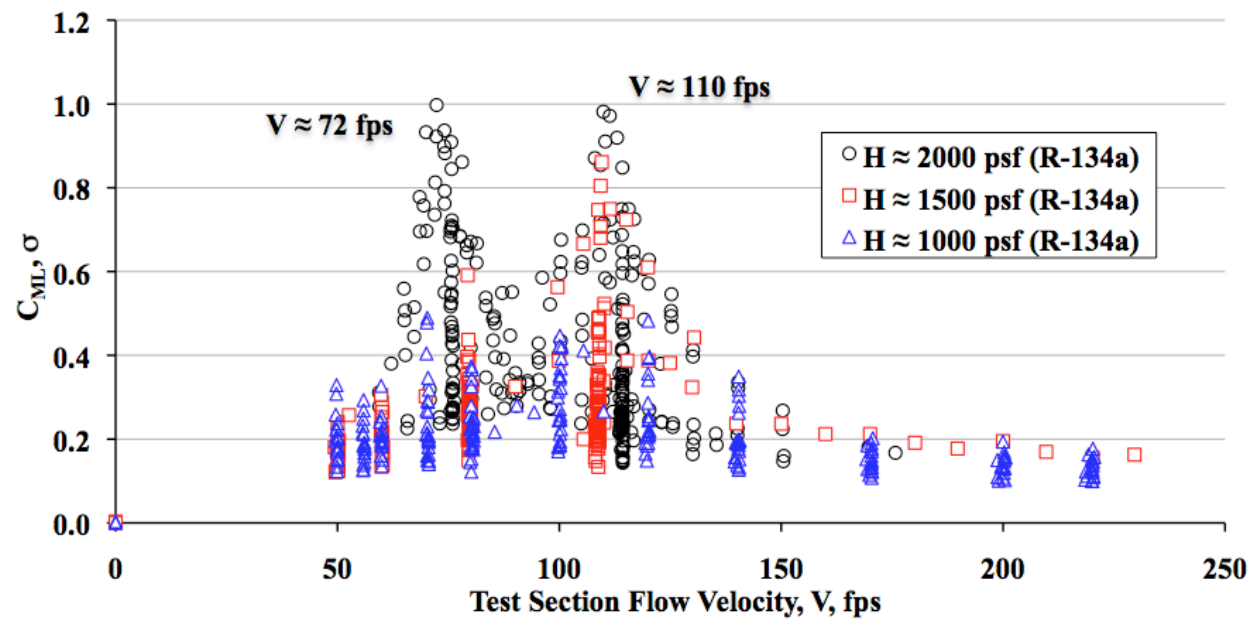

Figure 64. GWL Checkout Model. Base bending coefficient (rms) - lift direction. Velocity $=\mathbf{5 0}$ fps to 230 fps. Configuration \#2. 


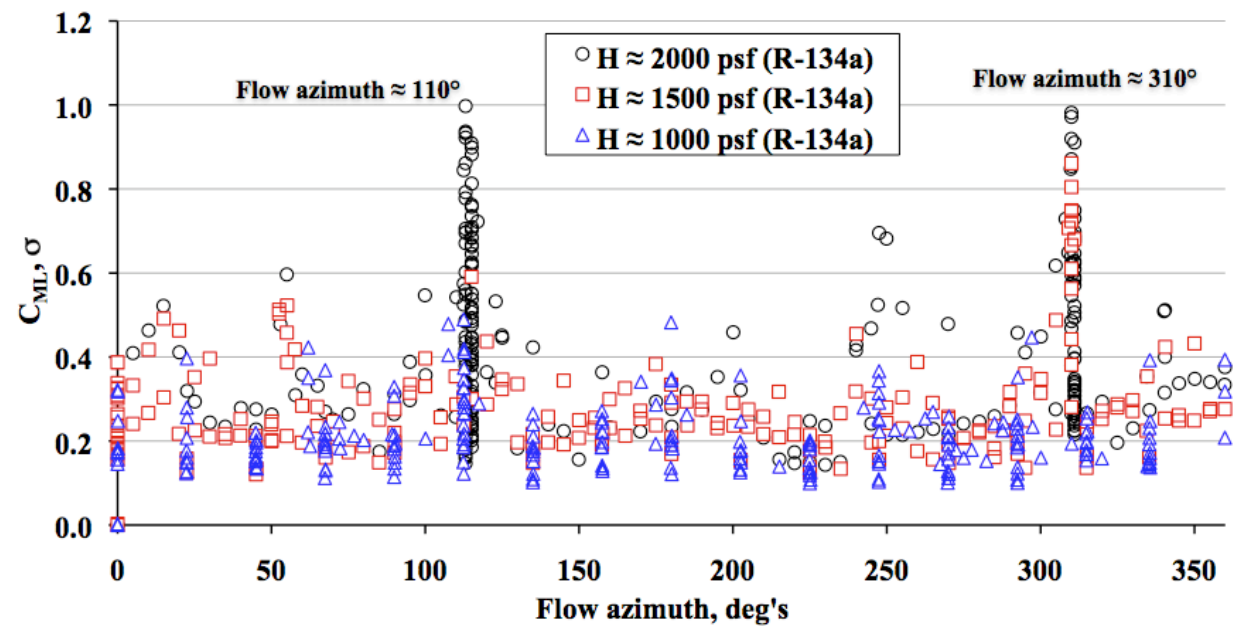

Figure 65. GWL Checkout Model. Base bending coefficient (rms) - lift direction. Flow azimuth $=0^{\circ}$ to $359^{\circ}$. Configuration \#2.

The data shown in Fig. 66 is a ratio of the $3 \sigma$ values for base bending moment due to lift over the mean values for base bending moment due to drag for a range of test flow velocities. These values give a quantitative indication of the ratio of peak dynamics in the lift direction with respect to steady drag. For example, a value of one indicates that the maximum dynamics in the lift direction were approximately equal to $+/-$ steady drag. The reader is also reminded that the wind tunnel model had a first bending mode damping of approximately $1.0 \%$ of critical. If the damping value had been lower, the magnitude of dynamic lift to steady drag would have been expected to be larger than shown. All data acquired in the wind tunnel was done with uniform flow, and the equivalent full-scale wind velocity values, $\mathrm{V}_{\mathrm{W}}$, shown in Fig. 67 for the same data, assumed uniform flow across the full-scale vehicle.

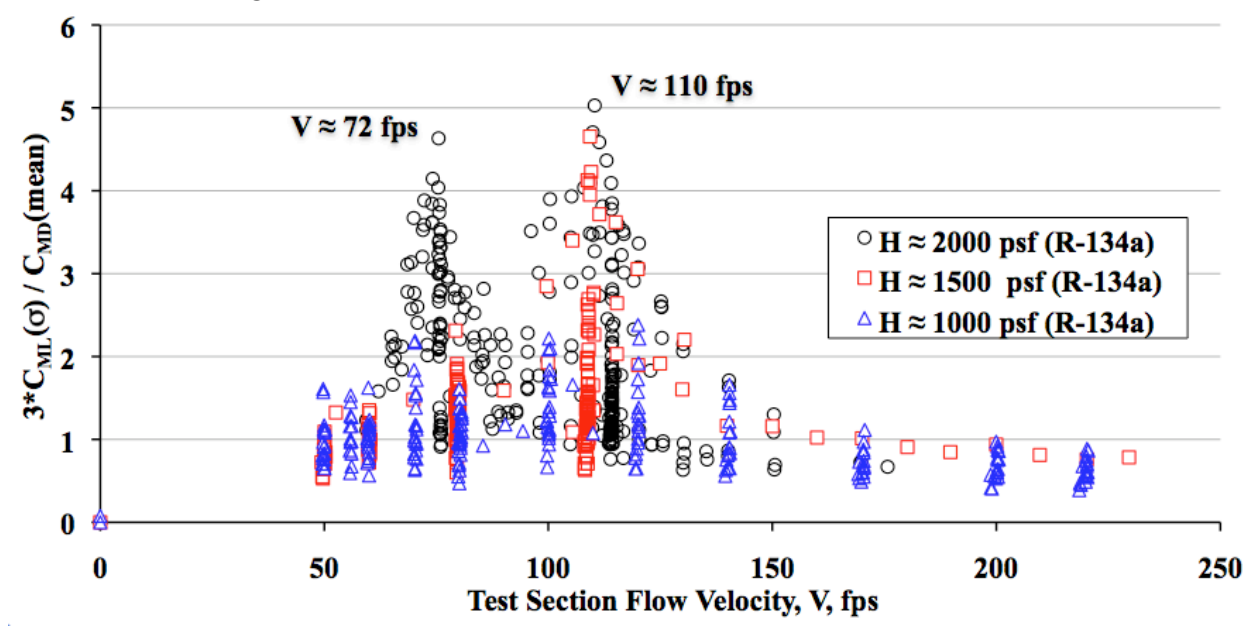

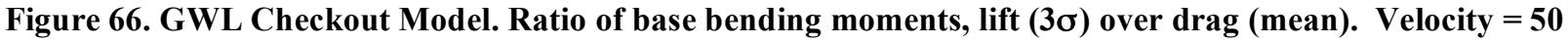
fps to 230 fps. Configuration \#2. 


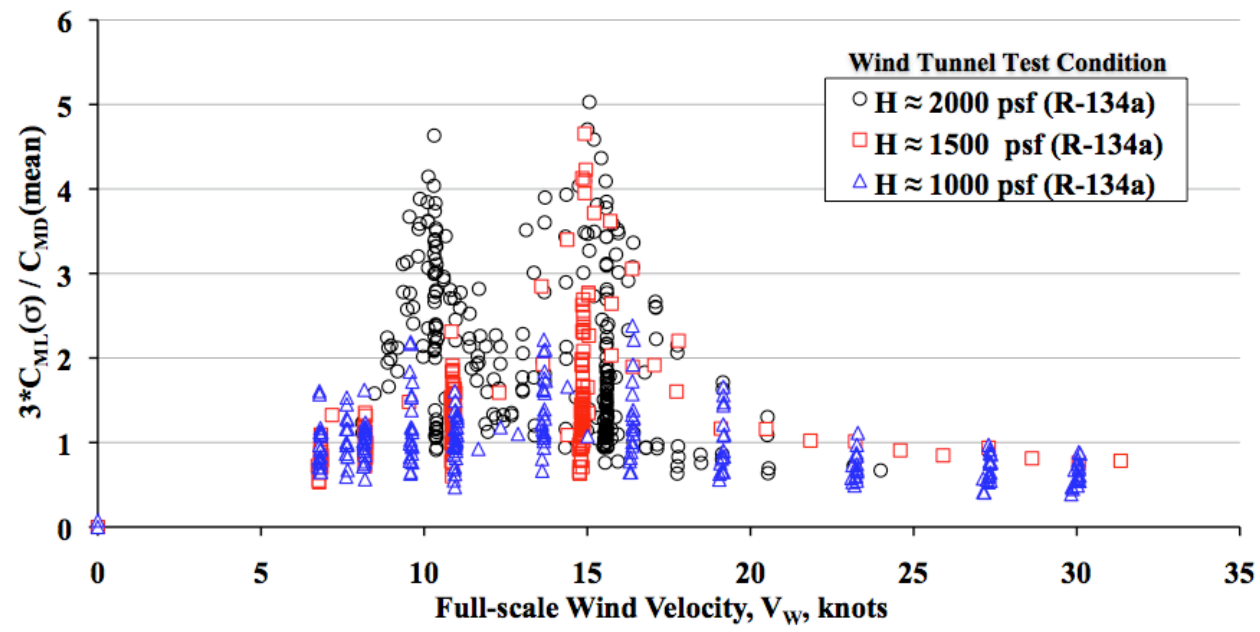

Figure 67. GWL Checkout Model. Ratio of base bending moments, lift $(3 \sigma)$ over drag (mean). $V_{W} \approx 6$ kts to 32 kts, uniform wind profile. Configuration \#2.

A question during the test and post test analysis concerned the portion(s) of the launch vehicle model experiencing the periodic vortex shedding that caused the peak responses in the test data. Based on the Strouhal and Reynolds numbers listed in Table 13, it was believed that the peak response at the flow velocity of $110 \mathrm{fps}$ was a result of strong vortex shedding that occurred on the upper stage of the vehicle model with a Strouhal number less than 0.20 caused by 3-D effects near the tip of the vehicle. Ref. 7 states that the tip shedding Strouhal number for structures with parallel sides or taper ratios great than 0.02 should be approximately 0.16 . It is theorized that the presence of the system tunnel protuberance on the upper stage increased the size of the effective diameter that produced a lower Strouhal number when calculated with the actual structural diameter of the upper stage. The source of the peak response at $72 \mathrm{fps}$ was more difficult to determine although the Strouhal and Reynolds numbers listed in Table 16 seemed to indicate that perhaps the lower portion of the upper stage was the more likely source with the higher Reynolds number and Strouhal number near 0.20. It was theorized that the flow in this region was more 2-D in nature.

Table 13. GWL Checkout Model. Strouhal and Reynolds numbers for primary WIO response peaks. Configuration \#2.

\begin{tabular}{|c|c|c|}
\hline Reference Stage & $\mathbf{V} \approx 72 \mathbf{f p s}$ & $\mathbf{V} \approx \mathbf{1 1 0} \mathbf{f p s}$ \\
\hline $1^{\text {st }}$ Stage & $\mathrm{Str} \approx 0.16, \operatorname{Re} \approx 1.45 \times 10^{6}$ & $\mathrm{Str} \approx 0.11, \mathrm{Re} \approx 2.22 \times 10^{6}$ \\
\hline Upper Stage & $\mathrm{Str} \approx 0.21, \mathrm{Re} \approx 1.89 \times 10^{6}$ & $\mathrm{Str} \approx 0.14, \mathrm{Re} \approx 2.88 \times 10^{6}$ \\
\hline
\end{tabular}

Dynamic base bending moment data due to lift showed that the peak response at 72 fps occurred at a different flow azimuth than the peak response at $110 \mathrm{fps}$. The WIO at $72 \mathrm{fps}$ occurred for a flow azimuth of approximately $110^{\circ}$ and the response at $110 \mathrm{fps}$ was for a flow azimuth of approximately $310^{\circ}$. This data is shown in Fig. 68 and Fig. 69, respectively. The figures also show the orientation of the model protuberances with respect to the flow angle. It seemed likely that the systems tunnel, on the first stage or upper stage, and the LH2 feedline on the upper stage significantly affected the flow azimuth at which these two peak responses occurred. Dynamic base bending moment due to lift data for these two flow azimuths at the highest Reynolds number conditions for Configuration \#2 are shown in Fig. 70 and Fig. 71. Peak responses did occur for configurations without protuberances installed but those occurred at somewhat different flow velocities and azimuths and at significantly lower response levels. 


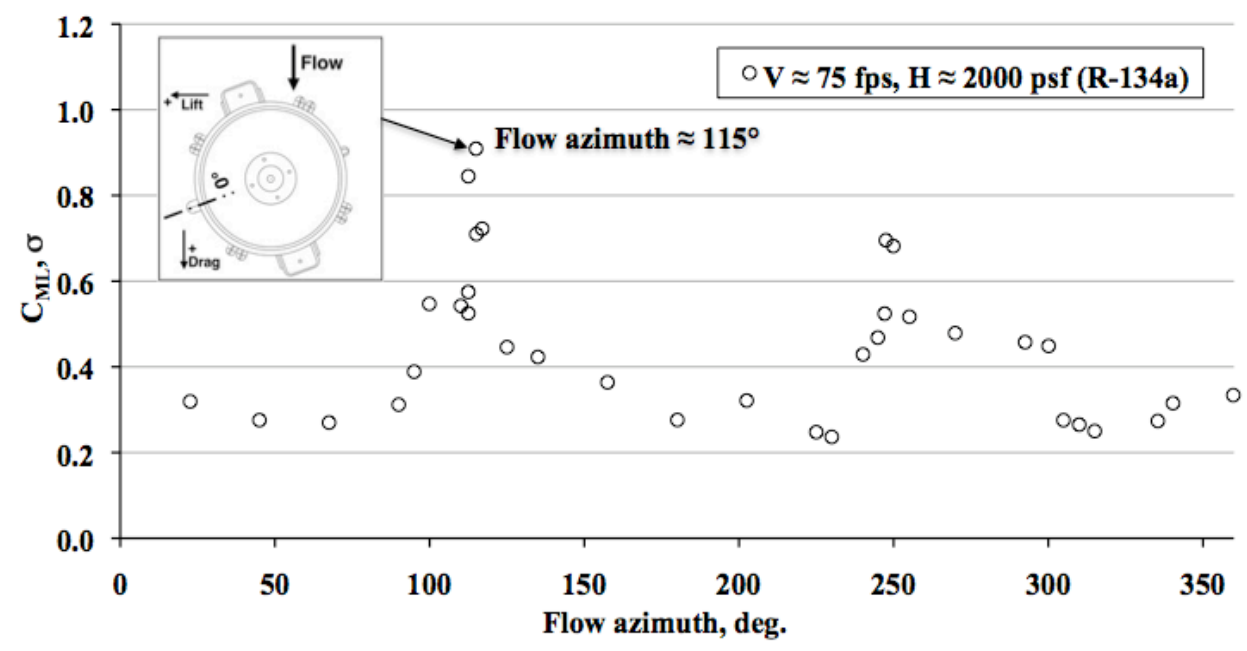

Figure 68. GWL Checkout Model. Effect of flow azimuth angle on base bending moment (rms) coefficient lift direction. Test section velocity $=75$ fps. Configuration \#2.

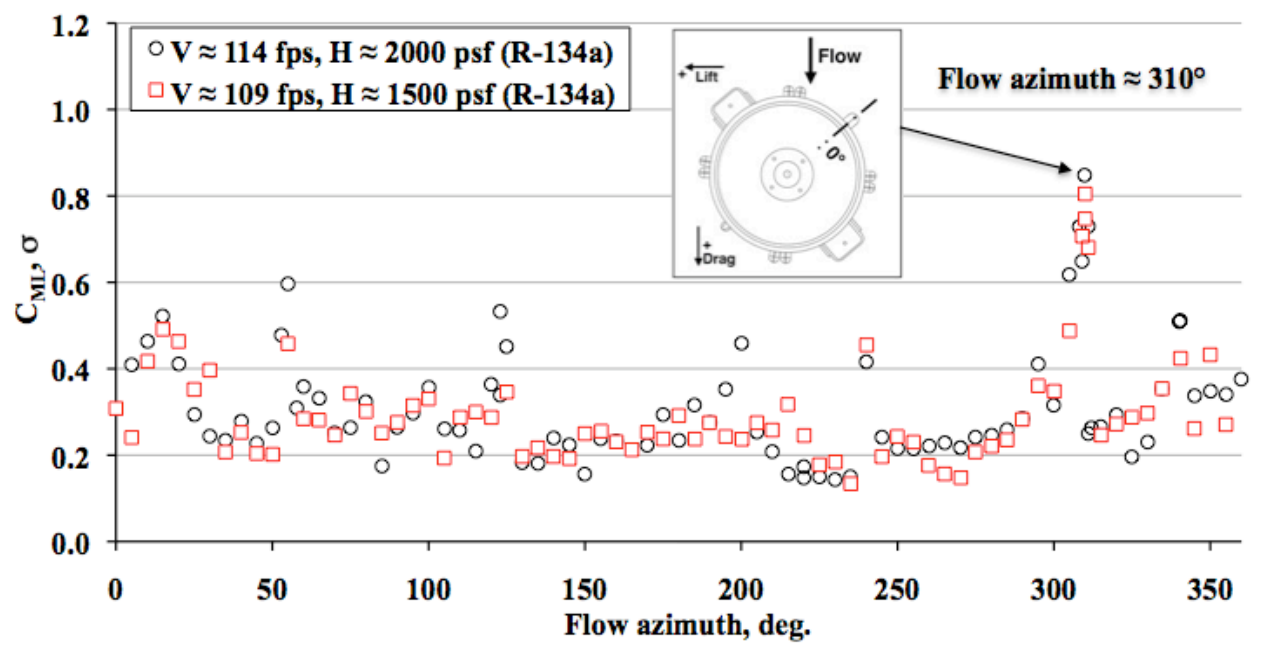

Figure 69. GWL Checkout Model. Effect of flow azimuth angle on base bending moment (rms) coefficient lift direction. Test section velocity $=109$ fps and 114 fps. Configuration \#2. 


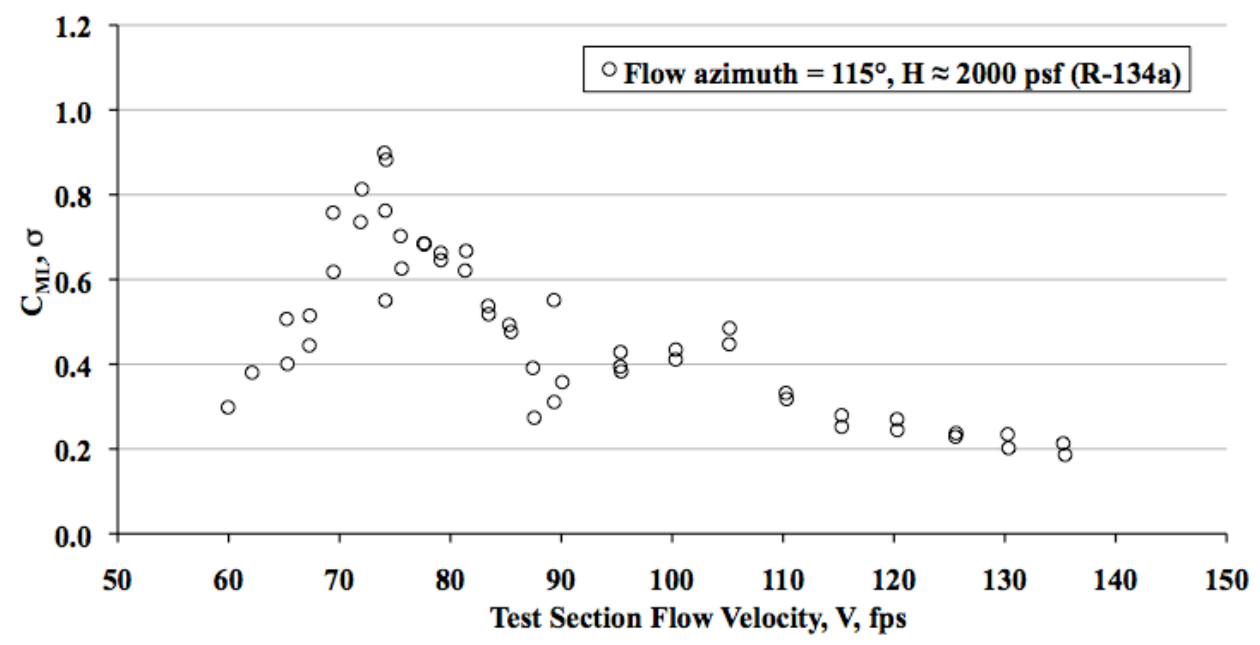

Figure 70. GWL Checkout Model. Effect of velocity on base bending moment (rms) coefficient - lift direction. Flow azimuth $=115^{\circ}$. Configuration $\# 2$.

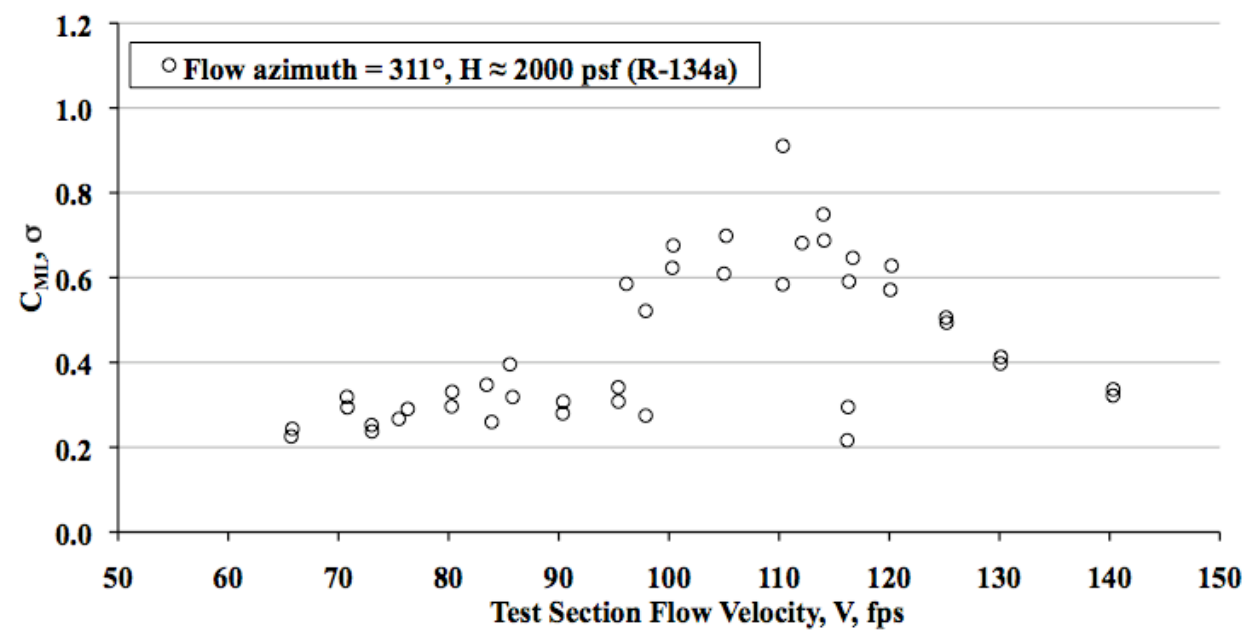

Figure 71. GWL Checkout Model. Effect of velocity on base bending moment (rms) coefficient - lift direction. Flow azimuth $=311^{\circ}$. Configuration \#2.

The base bending moment, as well as output from accelerometers, can be visualized with the base bending moment due to drag plotted as a function of the base bending moment due to lift. The example plot in Fig. 72 not only highlights the relation between bending moments due to lift and drag but also allows the reader to qualitatively see the magnitude of the dynamics by comparison to the steady (mean) bending moment values. During WIO and other periods of significant model response, the direction of predominant oscillation was offset in angle and not aligned with the lift direction. This offset angle of response is thought to have occurred as a result of a slightly weakened axis of vibration that existed for the model and resulted in orthogonal pairs of first and second bending modes. Due to this rotation, the dynamics in the drag direction may be slightly magnified due to a component of the lift dynamics acting in the drag direction. Overall, analyzing the details of a given data point in this manner, provided additional insight regarding the predominant direction of the dynamics. 


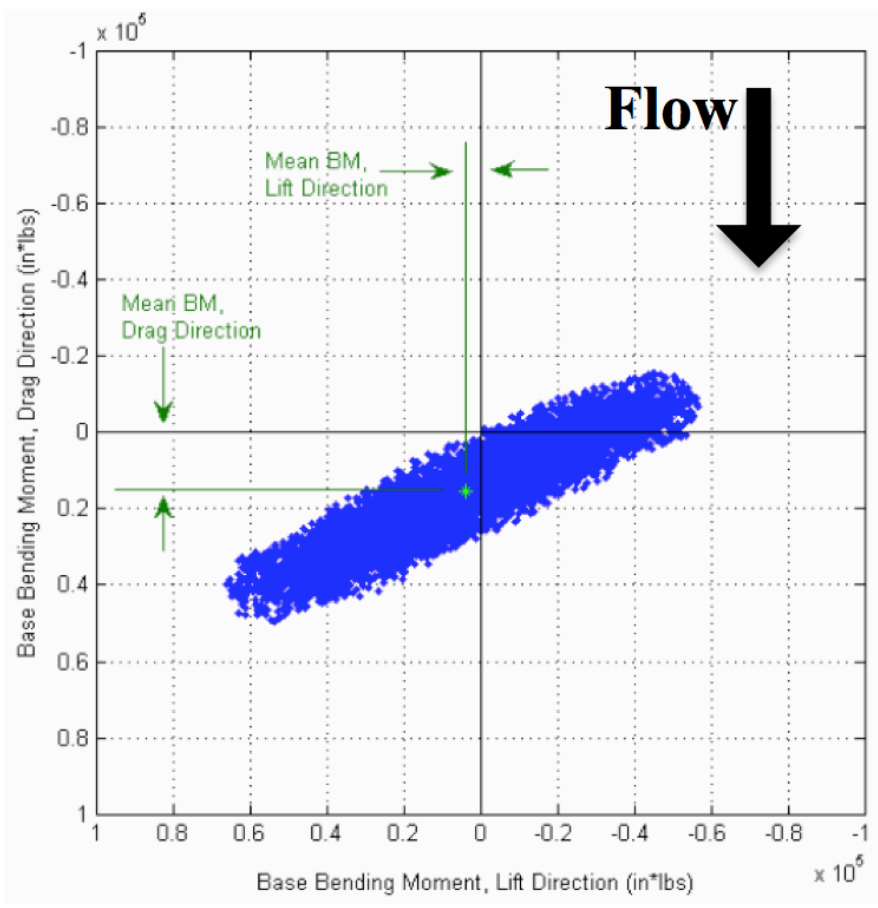

Figure 72. GWL Checkout Model. Base bending moment in lift and drag directions. Velocity $=110 \mathrm{fps}$. Flow azimuth $=311^{\circ} . H \approx 2000$ psf $(\mathbf{R}-134 a)$. Configuration \#2

Grit was added to the GWL Checkout Model for Configurations \#3 and \#4 to study the effect of surface roughness on model GWL and WIO characteristics. It was determined that the addition of grit to the upper stage decreased the dynamic response of the model at WIO peak response conditions. However, the size of the grit used for the GWL CM was later found to be much larger than recommended by Ref. 8 and applied to the model upper stage with questionable uniformity using double sided tape. This likely resulted in weakening of the strength and uniformity of the vortex shedding at these conditions and a corresponding reduction in model response at WIO conditions. Some test results did indicate that higher Reynolds flow could be simulated using grit and this method was refined and improved, and later used successfully on the Ares I-X GWL model.

The unsteady pressure transducers were installed on the GWL CM, in part, to provide data on the strength and frequency content of the unsteady aerodynamic forces acting on the model, specifically the vortices being shed that cause WIO. Overall, it was often difficult to identify vortex-shedding characteristics using the unsteady pressure transducers due perhaps to flow separation, transducer location, and the weak unsteady pressures at the vortex shedding frequency. Still, it should be noted that there were cases, such as for the peak responses for Configuration $\# 2$ at and around a velocity of $110 \mathrm{fps}$, where lock-in characteristics were observed in the PSD's for the unsteady pressures transducers located in the model upper stage. For these cases, the peak responses of the pressure transducer PSD's aligned with the structural frequency of the first bending mode response $(\approx 16 \mathrm{~Hz})$ not only at 110 fps but sometimes at velocities at much as 10 percent greater or less than the velocity for the peak response. As the velocity was further increased or decreased, the pressure PSD indicated the shedding frequency "unlocked' and the response data for the model would show a corresponding large decrease as the vortex shedding was no longer occurring at or near model resonant frequency.

\section{B. Ares I-X Ground Wind Loads Model}

\section{Test Summary}

The wind-on testing of the Ares I-X GWL model was more systematic and the test matrix more extensive than that conducted for the GWL CM. The initial configuration, rollout, was tested by conducting a velocity sweep, typically 50 to $200 \mathrm{fps}$ (model scale), for every $5^{\circ}$ of flow (wind) azimuth from 0 ' to $355^{\circ}$ except for one region around $90^{\circ}$ where the flow azimuth increment was $10^{\circ}$. Data was acquired at 5 fps or 10 fps increments in test section flow velocity that was equivalent to approximately 2 to 4 knots full-scale wind velocity. All data was acquired using heavy gas, R-134a, as the test medium at near atmospheric, highest possible Reynolds number, conditions. This provided a fairly dense test matrix covering the required design wind conditions for the Ares I-X 
FTV during rollout. As was done during the GWL CM test, additional data was acquired near regions of high model response and other test conditions of interest. Fig. 73 shows the test matrix, with full-scale values of wind velocity (uniform flow), in a polar coordinate system for the rollout configuration. The azimuthal coordinate represents wind azimuth and the radial coordinate represents velocity. Each circular symbol represents a wind azimuth and velocity combination for a data record. Repeat data points are represented by additional circular symbols for similar wind azimuths and velocity.

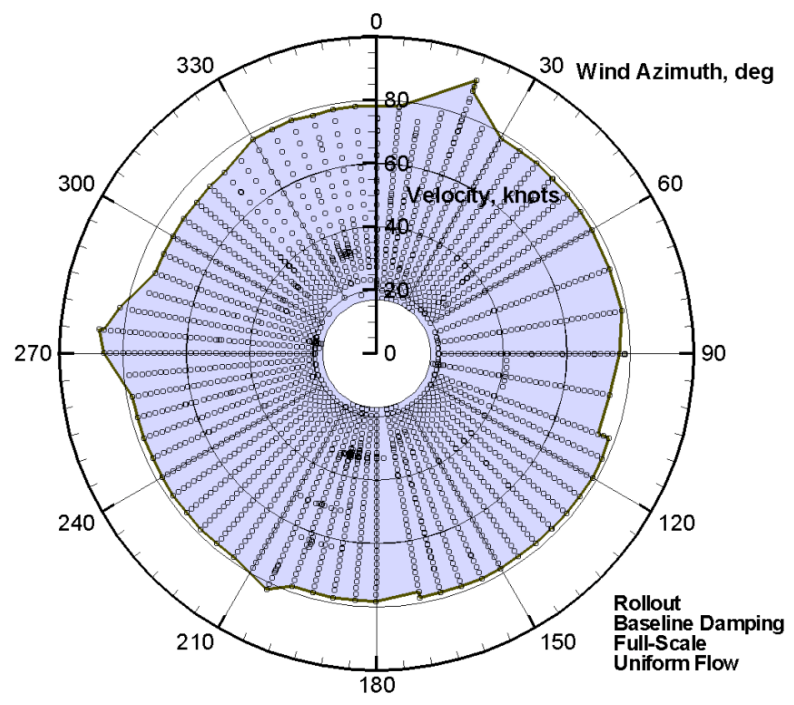

Figure 73. Ares 1-X GWL Model. Test matrix for rollout configuration. Full-scale values.

The on-pad configurations, stay and launch, generally had a full-scale velocity increment of four knots (10 fps model scale) and a wind (flow) azimuth increment of $10^{\circ}$. The test matrix for these configurations were generally coarser based on experience gained from rollout configuration testing and constraints on test schedule. The on-pad launch configuration was tested to much lower maximum wind velocities due to the lower wind requirements for this configuration. The on-pad stay and on-pad launch configuration test matrices are illustrated in Fig. 74 and Fig. 75 , respectively.

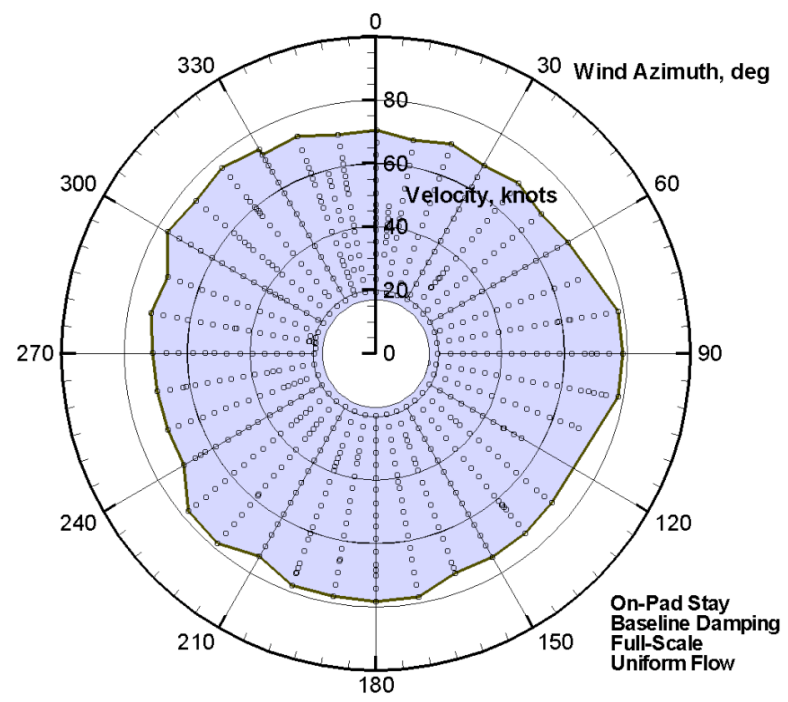

Figure 74. Ares 1-X GWL Model. Test Matrix for on-pad stay configuration. Full-scale values. 


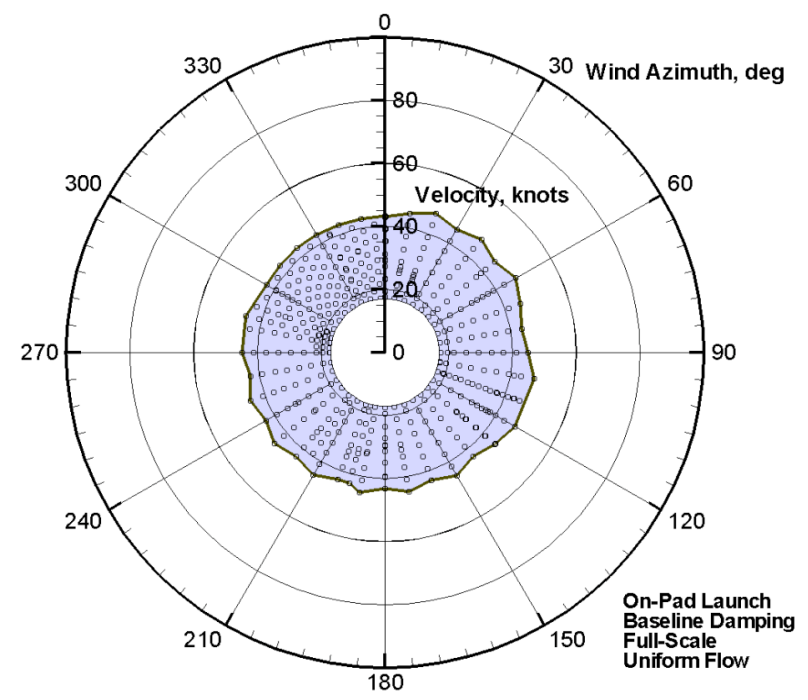

Figure 75. Ares 1-X GWL Model. Test Matrix for on-pad launch configuration. Full-scale values.

The final configuration, rollout with increased damping, was only run at select velocities and wind azimuth angles where high response was encountered earlier in the test for the rollout configuration with baseline damping. The test matrix for this configuration is presented in Fig. 76.

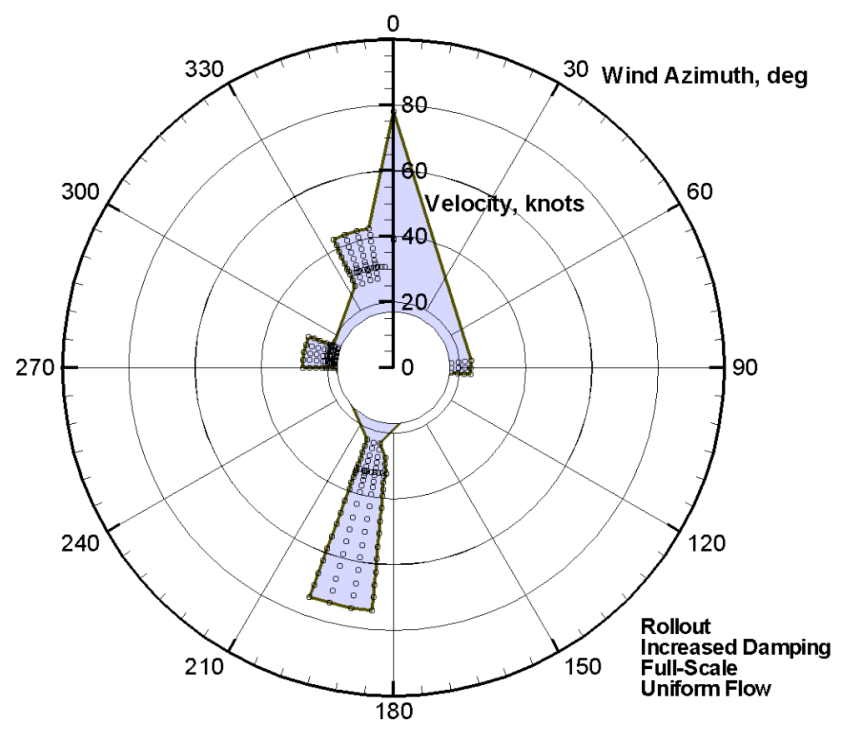

Figure 76. Ares 1-X GWL Model. Test Matrix for rollout configuration with damper. Full-scale values.

2. Modal Testing and Analysis

Modal testing of the Ares I-X GWL model in the rollout configuration (no damper installed) was performed in the TDT test section just prior to wind-on testing. The GWL TT was positioned at an angle of zero degrees (model azimuth angle of $180^{\circ}$ facing upstream) and suction applied to the turntable as it was during acquisition of all windon test data. An instrumented impact hammer was used to excite the model and response measured at 12 locations along the length of the launch vehicle model and balance in the $\mathrm{Y}$-axis or Z-axis direction, depending on direction of hammer impact. Results indicated that the damping for the first three vibration modes (1Y, $1 \mathrm{Z}$, and $2 \mathrm{Y})$ was below the target value of $0.50 \% \mathrm{Cr}$ and the damping of the fourth mode (2Z) was higher but still acceptable at $0.74 \% \mathrm{Cr}$. The measured frequencies for the four bending modes were all lower than target values predicted by the model-scale analytical model (FEM). Modal test frequency and damping results are presented in Table 14. The model measured frequencies for the first two bending modes agreed well with the full-scale target frequencies for the Ares I-X FTV on the MLP although the measured second bending mode frequencies were lower than the scaled target values. The 
lower than predicted measured frequencies were believed to be due primarily to the actual balance being less stiff than predicted by the analytical models. The effects of the turntable and tunnel test section floor stiffness were unknown and these were considered to be rigid in the analytical models. The measured modes shapes for all four bending modes correlated well with the model-scale predicted and scaled Ares I-X FTV target mode shapes and are presented in Fig. 77 and Fig. 78.

Table 14. Modal test results for Ares I-X GWL Model in TDT, GWL TT $=0^{\circ}$. Rollout configuration.

\begin{tabular}{|c|c|c|c|c|}
\hline Mode & Description & Frequency, Hz & Est. Damping, \%Cr & Node Point, MS \\
\hline 1 & 1st Bending, Y-axis & 6.69 & 0.46 & $\approx 175.14$ ” \\
\hline 2 & 1st Bending, Z-axis & 8.31 & 0.38 & $\approx 175.14$ ” \\
\hline 3 & 2nd Bending, Y-axis & 35.95 & 0.40 & 69.1 " \\
\hline 4 & 2nd Bending, Z-axis & 43.32 & 0.74 & 65.2 ” \\
\hline
\end{tabular}
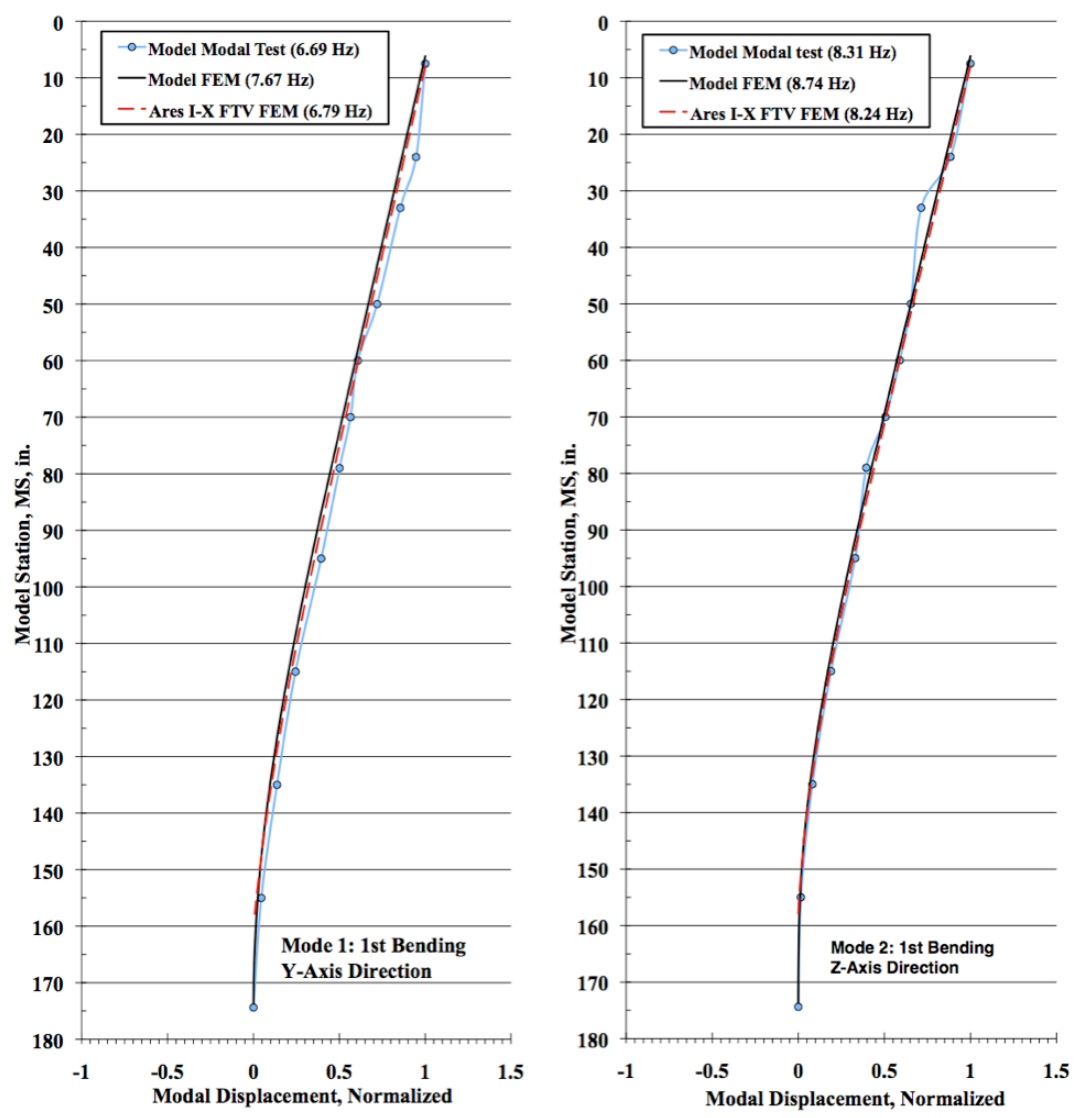

Figure 77. Ares I-X GWL Model modal test and analysis comparison for first bending modes, rollout configuration, in TDT. Model scale. 

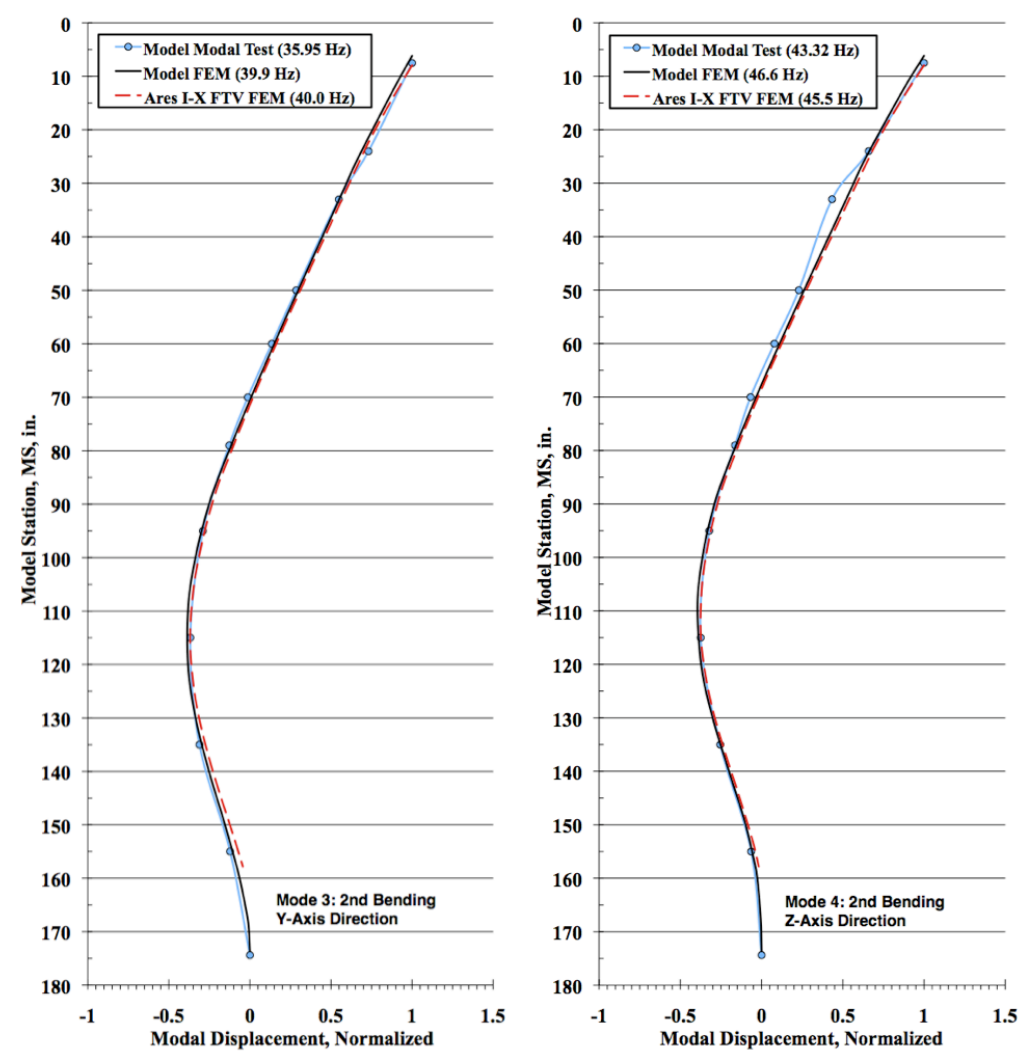

Figure 78. Ares I-X GWL Model modal test and analysis comparison for second bending modes, rollout configuration, in TDT. Model scale.

It was known from data acquired during the GWL CM test that the Ares I-X FTV model damping and frequencies could vary with turntable angle. Variation of vehicle model damping with turntable angle was determined during the Ares I-X GWL test using a large impact hammer with a hard rubber tip to excite the model in the model $\mathrm{Y}$-axis or Z-axis direction and recording model accelerometer response and decay data for the $1^{\text {st }}$ and $2^{\text {nd }}$

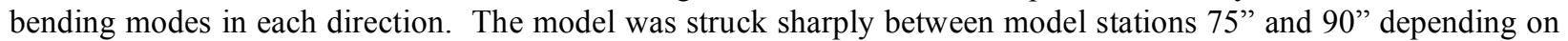
the mode that was targeted for excitation. Turntable angle was typically increased $10^{\circ}$ or $15^{\circ}$ between each set of hammer impacts until the model had been rotated in a full circle. This damping testing was performed several times during the wind-tunnel test to determine the average damping values for each of the test configurations and its variation with turntable angle. A summary of model damping with turntable angle for the four critical modes of vibration for the rollout configuration, no damper installed, is provided in Table 15.

Table 15. Modal test results for Ares I-X GWL Model in TDT, Rollout configuration.

\begin{tabular}{|c|c|c|c|}
\hline Mode & Description & Freq. Range, Hz. & Damping Range, \%Cr \\
\hline 1 (1Y) & 1st Bending, Y-axis & 6.7 & $0.24-0.28$ \\
\hline 2 (1Z) & 1st Bending, Z-axis & 8.3 & $0.22-0.42$ \\
\hline $3(2 \mathrm{Y})$ & 2nd Bending, Y-axis & $35.6-36.1$ & $0.33-0.60$ \\
\hline $4(2 \mathrm{Z})$ & 2nd Bending, Z-axis & $42.7-43.2$ & $0.77-1.35$ \\
\hline
\end{tabular}

Due to limited test-time, increased damping was tested for one model configuration (rollout) and only for a limited set of wind azimuth angles and velocities. An inspection of the data revealed that the modes $\mathrm{Z} 1$ and $\mathrm{Y} 2$ both encountered a significant WIO response in the rollout configuration with baseline damping. Since these responses occurred in different axes, the tuned-damper was configured to target both modes simultaneously.

A characteristic of most real structures is that damping is non-linear. As deflection increases damping typically increases. For the Ares I-X GWL wind tunnel model, the damping at extremely low amplitude was very low. As the amplitude increased, the damping increased until reaching a certain value and then remained relatively constant 
for a significant amplitude range. The amplitude range typically observed during testing was above the minimum amplitude where damping became constant. The presence of the tuned damper magnified this effect further. With the damper installed, it was observed that low amplitude vibrations did not posses enough acceleration to overcome the slider friction and allow the damper to be effective. Vibration amplitudes possessing the minimum required acceleration exhibited reasonably constant viscous damping. For the vast majority of data points, the dynamic acceleration was above the minimum required for damper effectiveness; therefore, the damping was considered constant with respect to amplitude.

The damper was tuned in the Y-axis to increase the damping in mode $2 \mathrm{Y}$ and the $\mathrm{Z}$-axis to increase the damping of mode 1Z. No attempt was made to increase the damping of modes $1 \mathrm{Y}$ or $2 \mathrm{Z}$. Table 16 shows the damping results of the vibration modes $1 \mathrm{Z}$ and $2 \mathrm{Y}$, expressed as a percent of critical. The target damping values for these modes were 1 percent of critical, and the slider mass was experimentally adjusted to reach the desired value. Because the frequencies of the model were slightly different during damper tuning, the damper was more effective than desired for modes $1 \mathrm{Z}$ and $2 \mathrm{Y}$ when the model was restored to the run configuration.

Table 16. Summary of average strucutral damping for Ares I-X GWL model in TDT. Rollout configurations.

\begin{tabular}{|c|c|c|c|c|c|c|c|c|}
\hline & \multicolumn{3}{|c|}{ Damping, \% Critical } & \multicolumn{3}{c|}{ Frequency (Hz, Model-Scale) } \\
\hline Mode & $\mathbf{1 Y}$ & $\mathbf{1 Z}$ & $\mathbf{2 Y}$ & $\mathbf{2 Z}$ & $\mathbf{1 Y}$ & $\mathbf{1 Z}$ & $\mathbf{2 Y}$ & $\mathbf{2 Z}$ \\
\hline \hline Baseline Average & 0.245 & 0.332 & 0.449 & 0.898 & 6.716 & 8.302 & 35.942 & 43.123 \\
\hline Damped Average & 0.283 & 1.831 & 1.476 & 0.985 & 6.695 & 8.260 & 35.660 & 43.438 \\
\hline
\end{tabular}

To better illustrate the effect of the damper upon modes $1 \mathrm{Z}$ and $2 \mathrm{Y}$, the time-history traces of acceleration for an accelerometer in the upper stage of the model for the Z-direction and Y-direction respectively are shown in Fig. 79 and Fig. 80. This data is compiled from separate hammer impacts acquired without the damper installed (baseline damping) and with the damper installed (increased damping). The time-histories were band-pass filtered to isolate the mode of interest. One should also note the difference in time scale between the figures since mode $2 \mathrm{Y}$ is significantly higher in frequency than mode $1 \mathrm{Z}$.

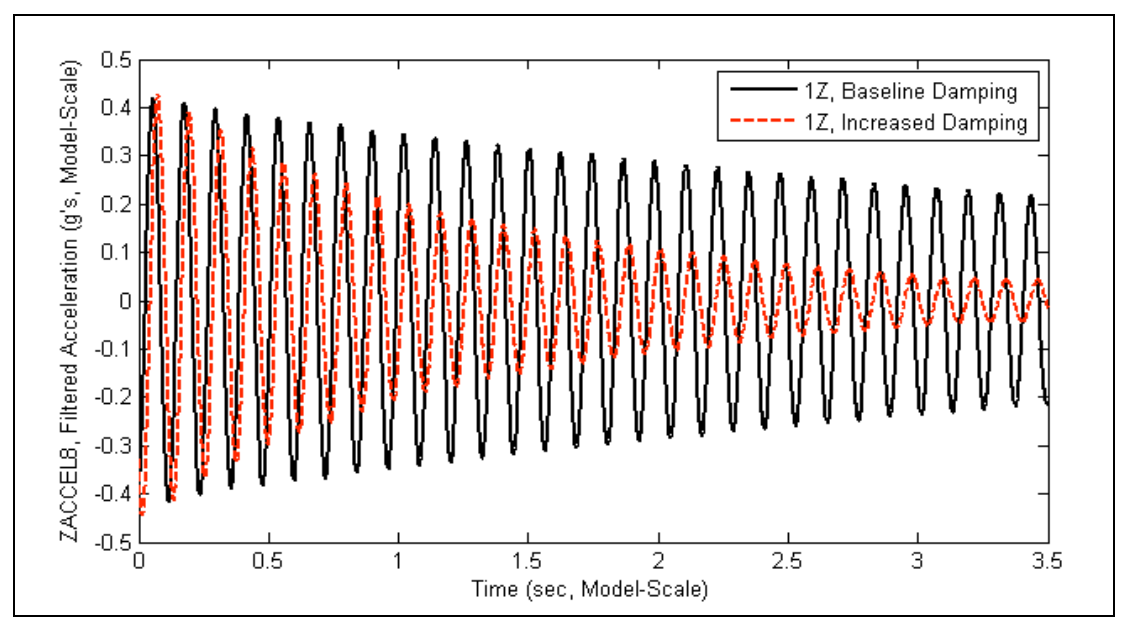

Figure 79. Ares I-X GWL Model. Effect of damper on mode $1 \mathrm{Z}$ during hammer impact. Time history of model accelerometer. Rollout configurations. 


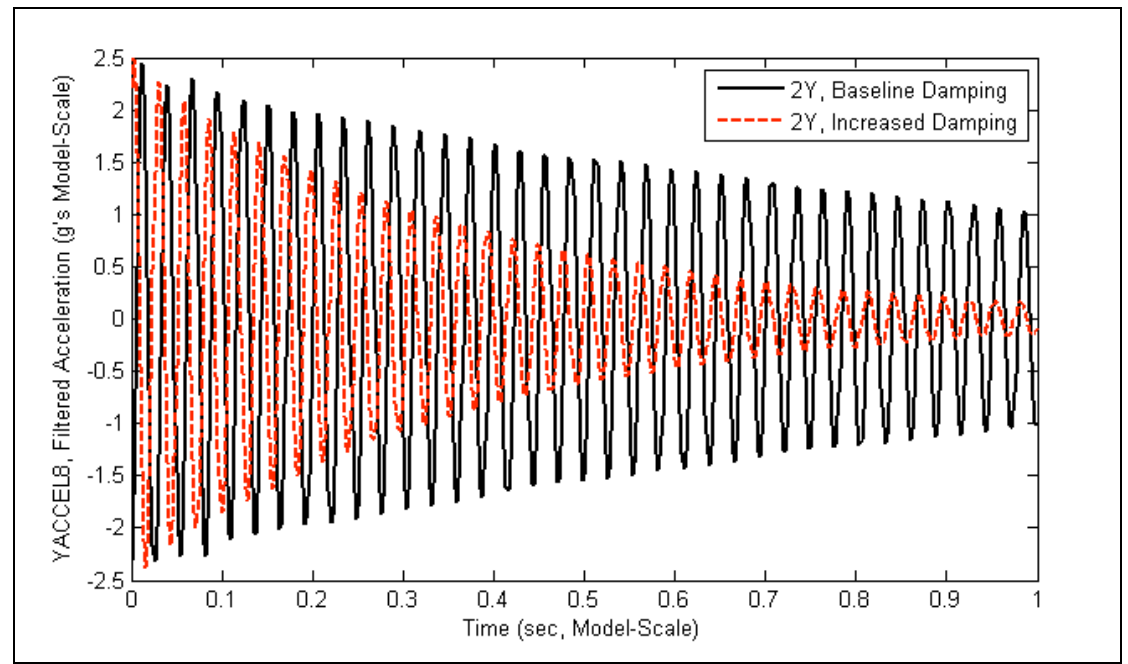

Figure 80. Ares I-X GWL Model. Effect of damper on mode 2Y during hammer impact. Time history of model accelerometer. Rollout configurations.

\section{Test}

As for the GWL CM test, the primary data from the Ares I-X GWL wind-tunnel test was the steady and dynamic base bending moment data since base bending moment data was important in determining critical loads at the base of, and along, the Ares I-X vehicle.

For each corresponding time step in the bending moment due to drag and the bending moment due to lift timehistory records, a root-sum-square of these components was calculated to determine the resultant load magnitude. The maximum value of the resultant load was extracted and is referred to in this paper as the "maximum resultant bending moment." The concept of maximum resultant bending moment is graphically represented in Fig. 3 . Thus, the maximum resultant bending moment is a scalar quantity and includes the combined effects of bending moment due to drag (a mostly steady component) and bending moment due to lift (a mostly dynamic component).

Plotted in Fig. 81 is the full-scale mean bending moment due to drag and the maximum resultant bending moment, both normalized, as a function of wind velocity for the rollout configuration. There were two wind velocities where classical wind induced oscillation (WIO) responses, characterized by large nearly sinusoidal response, were encountered. These WIO occurrences resulted in a large dynamic response of the fundamental bending mode in the $\mathrm{Z}$-axis (mode $1 \mathrm{Z}, \approx 0.2 \mathrm{~Hz}$ full-scale) for a full-scale wind velocity of around $19 \mathrm{knots}$ and a wind azimuth of $280^{\circ}$, and of the second bending mode in the $\mathrm{Y}$-axis (mode $2 \mathrm{Y}, \approx 1.0 \mathrm{~Hz}$ full-scale) at a full-scale wind velocity of around 33 knots for wind azimuths of approximately $195^{\circ}$ and $345^{\circ}$. It is believed that the large first bending mode WIO response encountered at $280^{\circ}$ wind azimuth was caused by tip vortex shedding effects as described in Ref. 7(SSS-G). Additionally, it is important to note that the Command/Service Module umbilical protuberance was near the tip of the vehicle and oriented nearly orthogonal to the flow direction for a wind azimuth angle of $280^{\circ}$. Therefore, it was deduced that this large response was likely a result of a Command/Service Module umbilical protuberance effect on tip shedding. The second bending mode WIO response was almost certainly the result of strong first stage vortex shedding occurring at a frequency of around $36 \mathrm{~Hz}$ (model-scale) that excited mode $2 \mathrm{Y}$ with a resultant Strouhal number of approximately 0.21 . It should be noted that, as a result of the aeroelastic scaling relationships, the wind tunnel idle speed represented a full-scale velocity of approximately18 knots. Transient data acquired during tunnel startup and shutdown sequences suggest that regions of significant first mode WIO response existed at wind velocities below this test limitation of 18 knots but are not presented in this paper. 


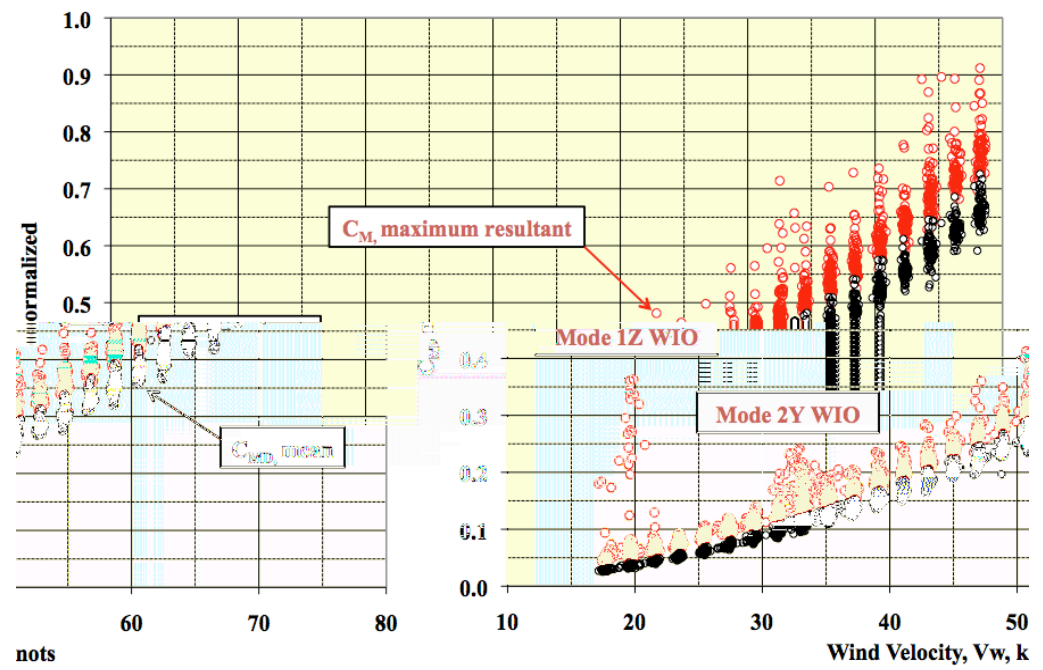

Figure 81. Ares I-X GWL Model. Mean bending moment due to drag and maximum resultant bending moment. Rollout configuration. Full-scale normalized values, uniform flow.

As discussed earlier, vortex shedding characteristics are dependent on Reynolds number and grit was applied to the Ares I-X FTV model to accelerate flow transition to simulate full-scale Reynolds (full-scale) transcritical and supercritical flow conditions. An indication of transition to supercritical flow is an increase and then leveling off of drag coefficient ${ }^{10,11}$. Fig. 82 shows a plot of mean drag coefficient versus test section flow velocity for the Ares I-X GWL model in the rollout configuration. The sharp change in slope of the plot at approximately $80 \mathrm{fps}(\approx 30$ knots full-scale), typical for the model in the rollout configuration, was theorized to be where the flow had fully transitioned to supercritical flow. Above this velocity, vortex shedding and resultant WIO response should have been reasonably unaffected by test section turbulence characteristics. This was important since the primary WIO and GWL results of interest were at velocities above $80 \mathrm{fps}$, model scale. Results below this velocity were viewed with less certainty as to simulating full-scale Ares I-X FTV WIO and GWL characteristics.

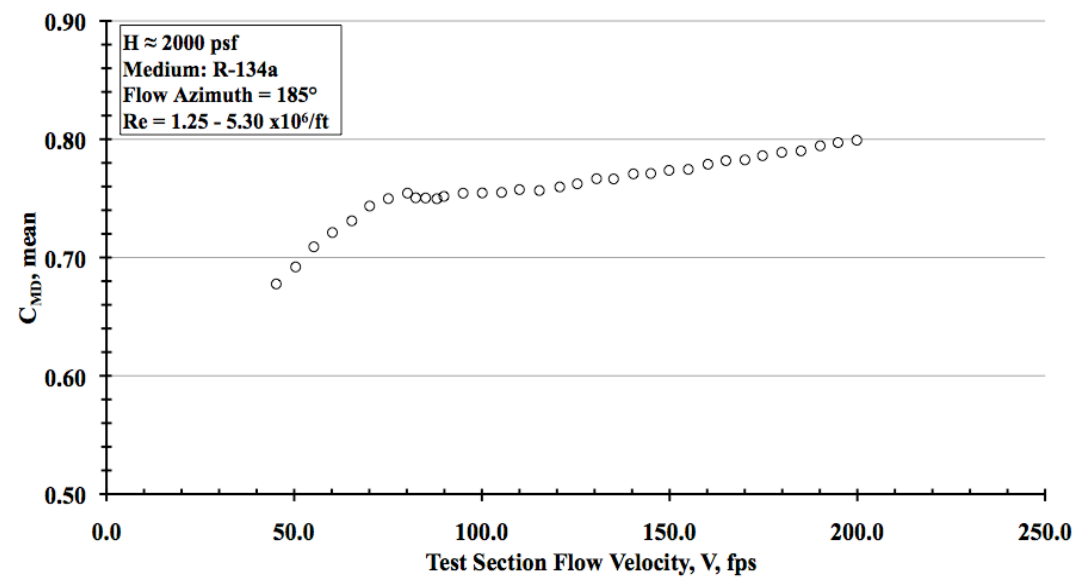

Figure 82. Ares I-X GWL Model. Base bending moment (mean) coefficient, due to drag, rollout configuration. Velocity sweep at flow angle $=185^{\circ}$. Model-scale results.

As discussed earlier, a band-pass filter was applied to the time history of bending moment due to lift in order to isolate the dynamic contribution of either the first bending modes (modes $1 \mathrm{Y}$ and $1 \mathrm{Z}$ ) or the second bending modes (modes $2 \mathrm{Y}$ and 2Z). The standard deviation of these filtered components were compared to the standard deviation of the unfiltered signal in order evaluate the magnitude of dynamic load resulting from vibration of these modes. Fig. 83 presents the normalized standard deviation of the first and second modes for the rollout configuration and shows that the WIO response peak around 19 knots was the result of the first bending modes responding to vortex shedding and the peak at 33 knots the result of second bending mode response. Model dynamic loads at higher wind 
velocities for the rollout configuration were primarily due to first bending mode response although these were not considered WIO as large single frequency sinusoidal motion was not observed on the model. For the entire Ares I-X GWL Model test, on average, 96 percent of the dynamic loads could be attributed to the modes 1Y, 1Z, 2Y and $2 \mathrm{Z}$. This allowed the dynamic loads to be modeled as a linear combination of individual modal responses for all test configurations and nearly all test conditions.

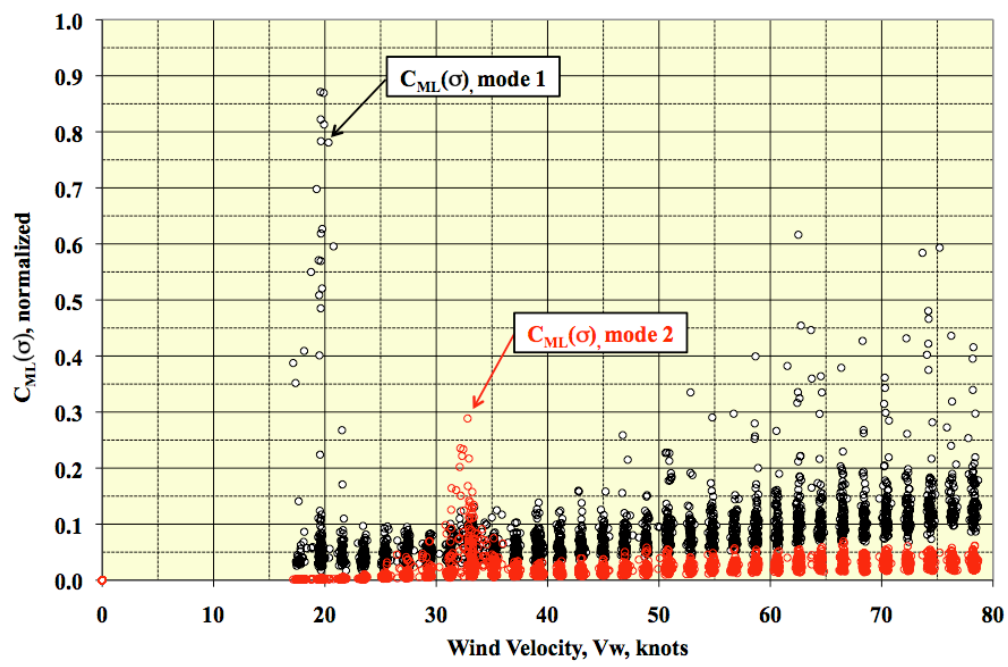

Figure 83. Ares I-X GWL Model. Contribution of 1 st and 2 bending modes to dynamic bending moment in lift direction. Full-scale normalized values, uniform flow.

Fig. 84 contains the mean bending moment due to drag and the maximum resultant bending moment as a function of wind velocity for the on-pad stay configuration. No WIO was observed for this configuration at all tested wind speeds and azimuths. There was an unexplained occurrence of significant first mode response at high velocity. This was not unexpected as the close proximity of the FSS and RSS were expected to significantly interfere with the flow around the vehicle. As expected there is more scatter in the mean bending moment due to drag data as a function of wind azimuth angle with the addition of the FSS and RSS in comparison to the rollout configuration.

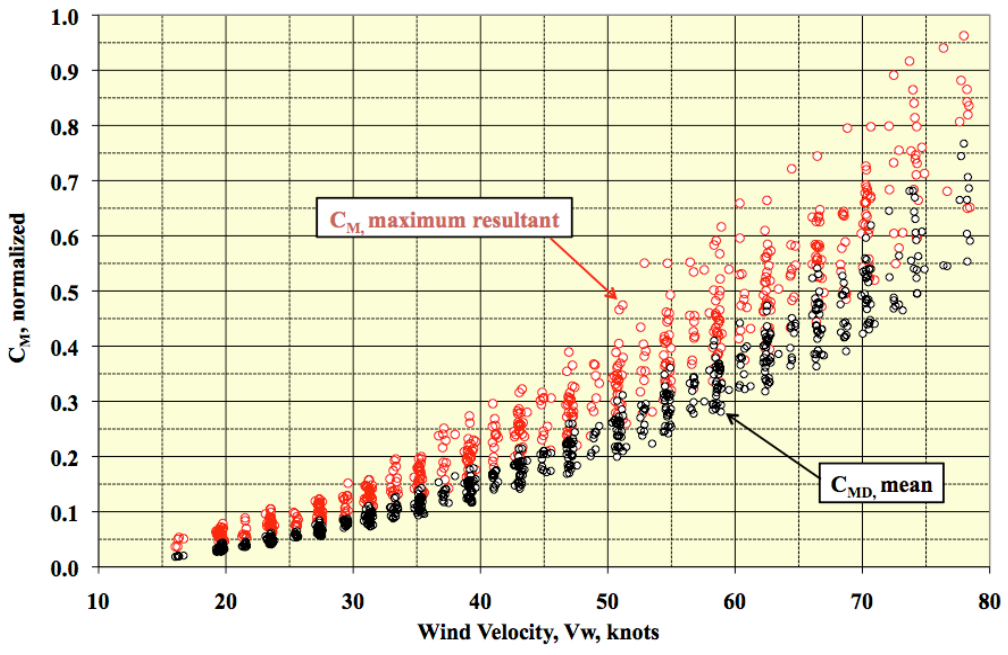

Figure 84. Ares I-X GWL Model. Mean bending moment due to drag and maximum resultant bending moment, on-pad stay configuration. Full-scale normalized values, uniform flow. 
Fig. 85 contains the mean bending moment due to drag and the maximum resultant bending moment as a function of wind velocity for the on-pad launch configuration. The test matrix for the on-pad launch configuration, as discussed earlier, was limited to lower wind velocities since the Ares I-X FTV had significant wind restrictions associated with launch and testing to higher velocities was considered unnecessary. As for the on-pad stay configuration, no WIO was observed for the on-pad launch configuration. Dynamic oscillations of the model were, on average, higher with the FSS and RSS present in the on-pad stay and on-pad launch configurations in comparison to the rollout configuration. Certain wind azimuth angles and velocities yielded a significant increase in dynamic response and the loads associated with the second bending mode frequencies (modes $2 \mathrm{Y}$ and $2 \mathrm{Z}$ ) were several times larger than those acquired for the rollout configuration.

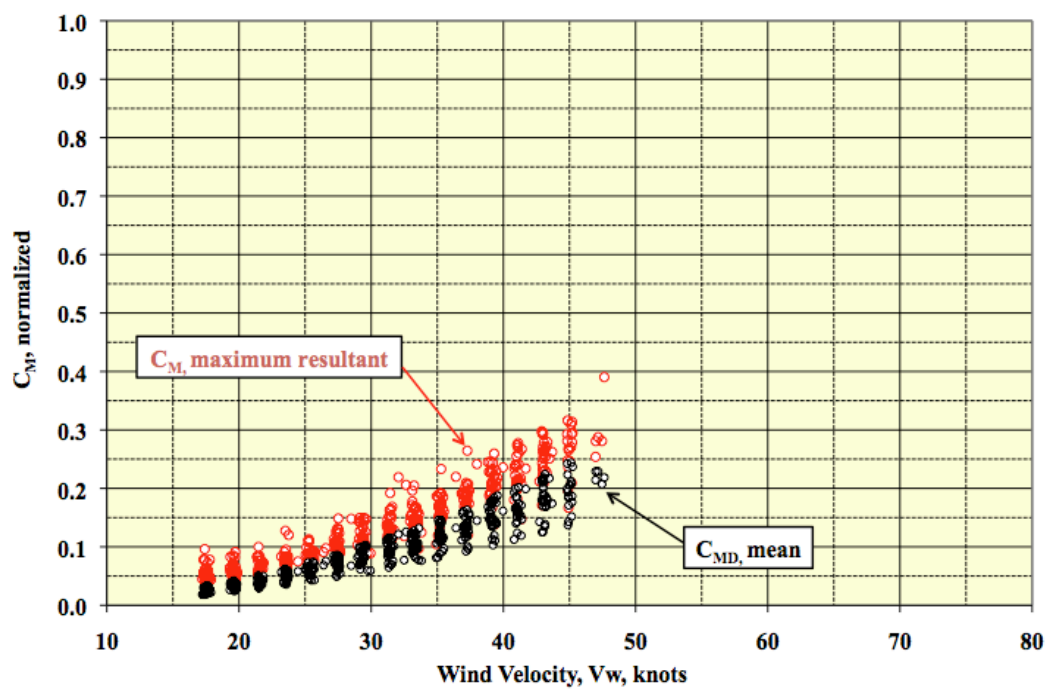

Figure 85. Ares I-X GWL Model. Mean bending moment due to drag and maximum resultant benidng moment, on-pad launch configuration. Full-scale normalized values, uniform flow.

Finally, Fig. 86 contains the mean bending moment due to drag and the maximum resultant bending moment as a function of wind velocity for the rollout configuration with increased damping. There is considerably less data for this configuration with the damper present than for the rollout configuration with baseline damping. Only select wind azimuth angles and wind velocities were run, and, in general, the model response dynamics were considerably lower with the presence of the internal damper. These results were consistent with the findings of previous studies, the inclusion of an internal damper reduces the dynamic response resulting from WIO. If the structural damping is increased above a threshold value, the dynamic response is reduced to insignificant values (compared to mean loads) and is representative of a structural response to random input. 


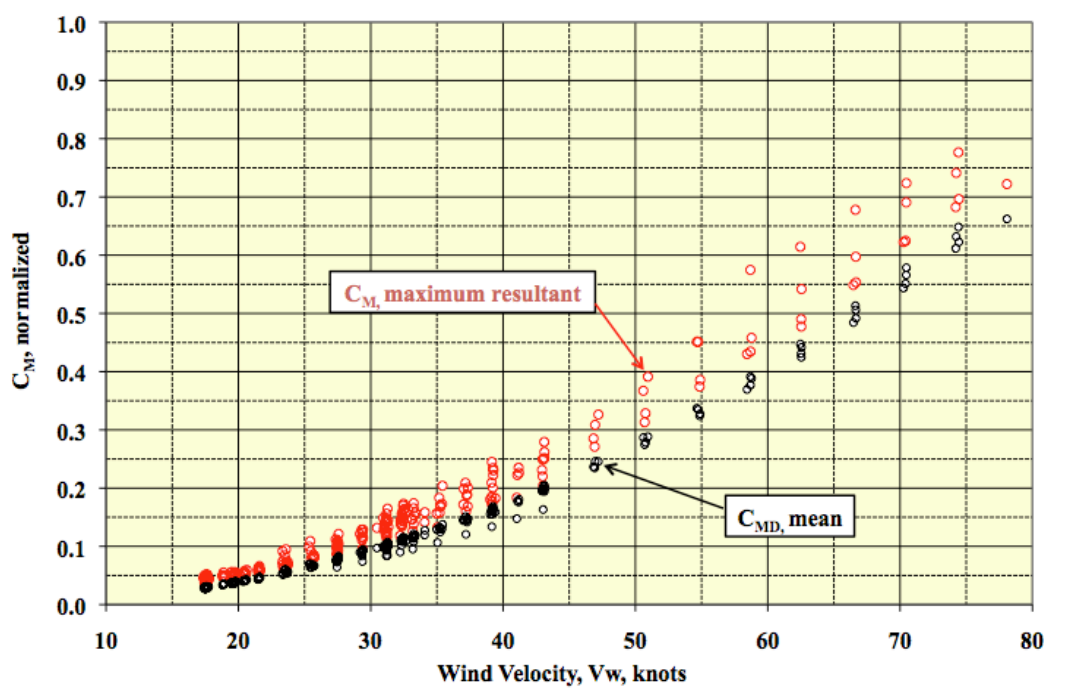

Figure 86. Ares I-X GWL Model. Mean bending moment due to drag and maximum resultant benidng moment, rollout configuration with increased damping. Full-scale normalized values, uniform flow.

As a result of the aeroelastic scaling equations and experimental limitations, first bending mode WIO responses were not expected within the wind tunnel velocity envelope. However, a strong first mode WIO response was observed at a wind azimuth angle of $280^{\circ}$ and a velocity of approximately 19 knots full-scale and correlated to a Strouhal number of approximately 0.13 based upon upper stage diameter.

Shown in Fig. 87 are response envelope plots, full-scale values, of the bending moments in the wind-axis and model-axis coordinate systems for this first mode WIO response. The axes directions have been reversed where applicable such that the displacement of the response envelope corresponds to the load direction, as viewed from above, in the respective axis system. The envelope plot for this response illustrates how the launch vehicle model did not respond perpendicular to the flow, in the lift direction, but aligned with the primary axis of mode $1 \mathrm{Z}$. This was typical for the WIO observed during the Ares I-X GWL Model and GWL CM tests.

Plotted in Fig. 88 is a portion of the time-history signal of bending moment due to lift and the corresponding power spectral density function, converted to equivalent full-scale values for this data record. As shown in the time history, the bending moment response is sinusoidal with nearly constant amplitude.
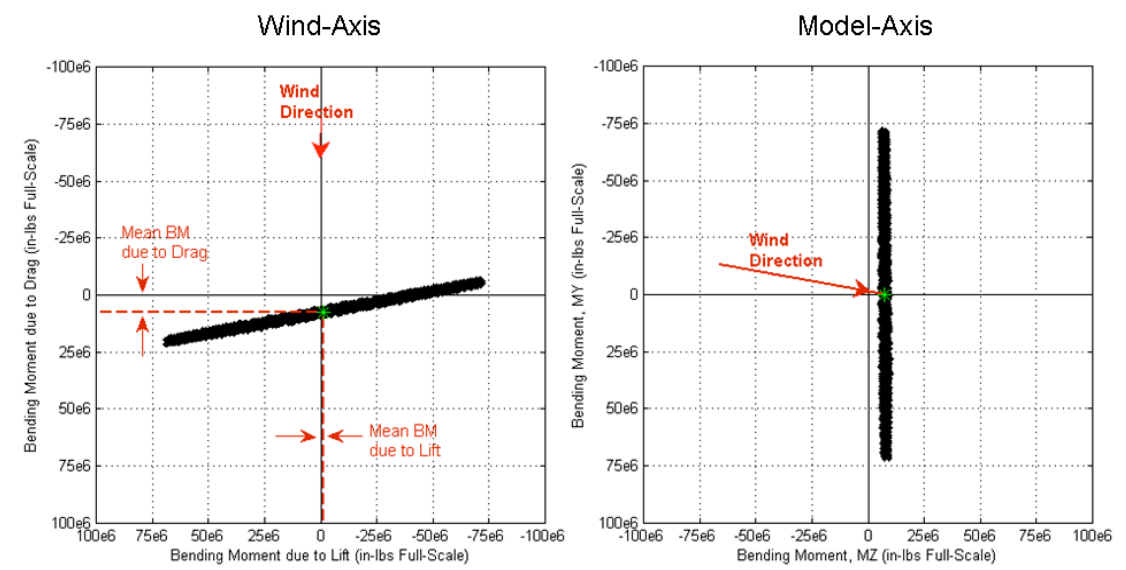

Figure 87. Ares I-X GWL Model. Response envelope plots of mode $1 Z$ WIO response at wind azimuth of $280^{\circ}, 19$ knots full-scale, rollout configuration. Full-scale values. 

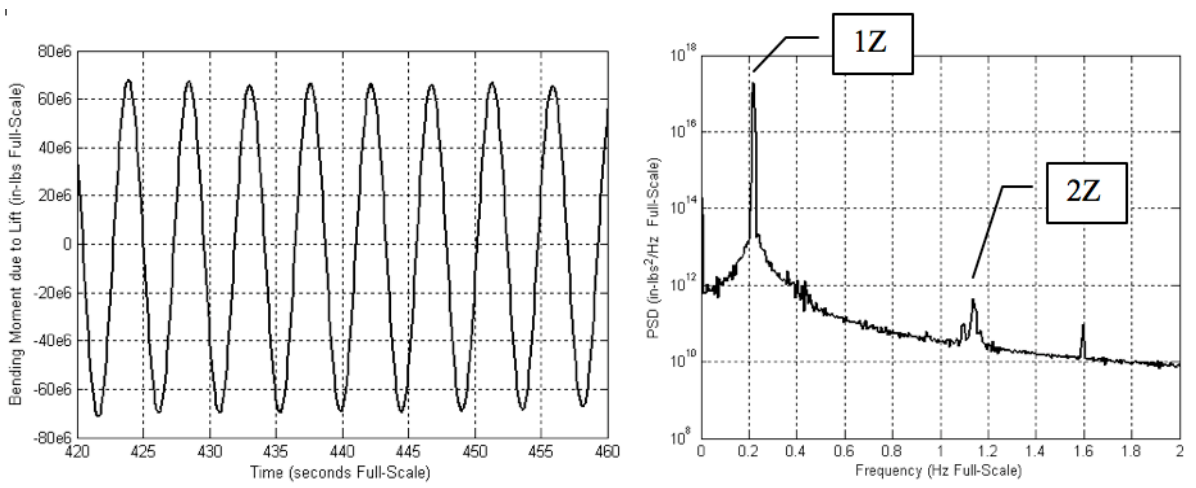

Figure 88. Ares I-X GWL Model. Bending moment due to lift time history and PSD for first mode WIO response (mode $1 \mathrm{Z})$ at $280.1^{\circ}$ and 19 knots; rollout configuration. Full-scale values.

A strong response of the second bending mode in the $\mathrm{Y}$-axis (mode 2Y) was encountered for the rollout configuration at a wind azimuth angle of $195^{\circ}$ and a velocity of 33 knots full-scale. At this particular condition, the vibration of the model was nearly sinusoidal in mode $2 \mathrm{Y}$ with a small contribution of mode 1 Y. At velocities only two knots above or below the peak excitation velocity, however, the response of the model was more random in amplitude and involved significant contributions of multiple vibration modes. Depicted in Fig. 89 are response envelope plots of the bending moments in the wind-axis and model-axis coordinate systems for this second mode WIO response. As for the first bending moment WIO discussed above, the envelope plot for this response illustrates how the model does not respond perpendicular to the flow, in the lift direction, but aligned with the primary axis of mode $2 Y$.

Plotted in Fig. 90 is a portion of the corresponding time-history signal of bending moment due to lift and the corresponding power spectral density function, converted to equivalent full-scale values for this second mode WIO response. The plots shown in Fig. 89 and Fig. 90 are in the same scale as those presented for the first mode WIO response. One can clearly observe the increase in frequency, the presence of a lower frequency response, and the decrease in amplitude in the time-history response by comparison to the first mode WIO response.

Even though the base bending moment magnitude of the second mode WIO response was less than the base bending moment magnitude of the first mode WIO response, the second mode response may contain more critical loading. As previously mentioned, the load distribution was significantly different for a second mode displacement than for a first mode displacement, and loads may have been higher at vehicle locations other than the base where load limits also tended to be lower.
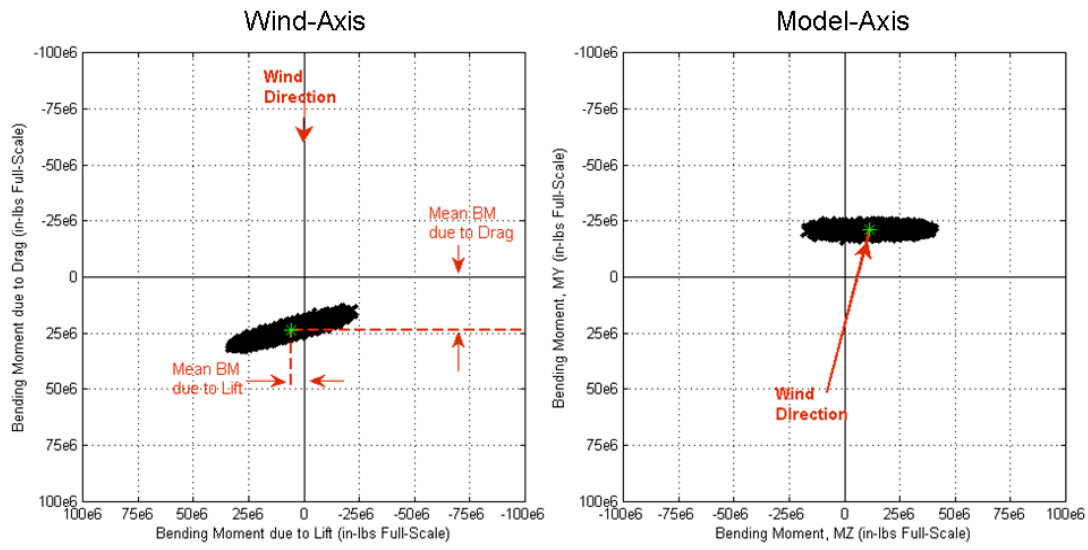

Figure 89. Response envelope plots of mode 2 Y WIO response at wind azimuth of $195^{\circ}, 33$ knots full-scale; rollout configuration. 

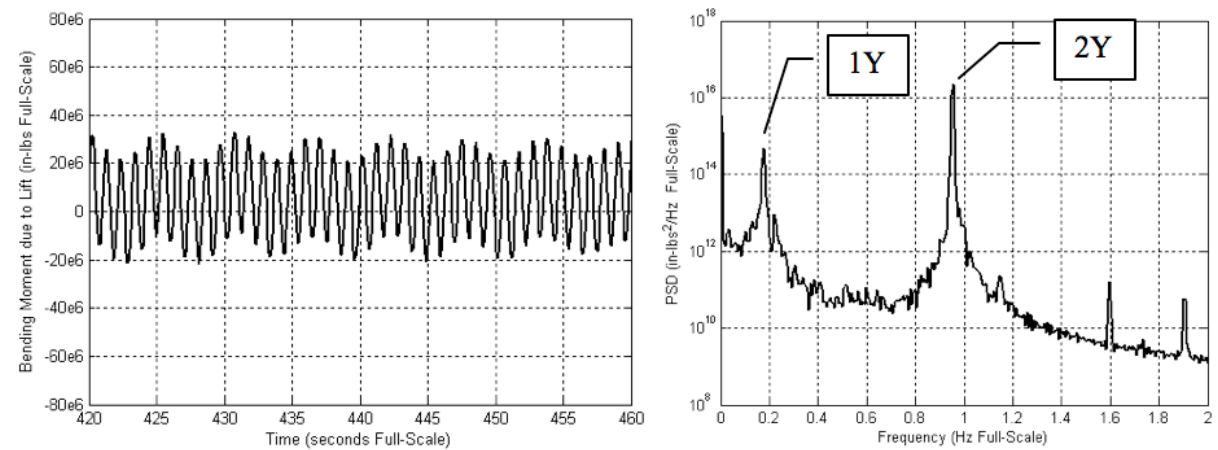

Figure 90. Bending moment due to lift time history and PSD for second mode WIO response (mode 2 Y) at $195^{\circ}$ and 33 knots; rollout configuration.

Drag forces on the Ares I-X FTV during rollout and just prior to launch were considered critical for vehicle structural integrity and launch criterion, respectively. Fig 91 is a color contour plot in polar coordinates of the normalized mean base bending moment coefficient due to drag for the rollout configuration.

With the exception of the Command/Service Module umbilical protuberance, LAS raceway, and booster tumble motors, the model was structurally and geometrically symmetrical about the $\mathrm{X}-\mathrm{Y}$ plane (east-west). The data presented in Fig. 91 was also approximately symmetrical, and the regions of increased drag were believed caused by separation around the upper stage and first stage systems tunnels (located at a wind azimuth angle of $270^{\circ}$ ) and the LH2 feed line (located at a wind azimuth angle of $90^{\circ}$ ). Values for mean bending moment coefficients due to drag (drag coefficients) for the rollout configuration were typically between 0.60 and 0.80 for most wind speed and azimuth conditions.

Similarly, Fig. 92 is a contour plot in polar coordinates of the mean base bending moment coefficient due to drag for the on-pad launch configuration. With the inclusion of the FSS and RSS, this model configuration was highly unsymmetrical with respect to wind azimuth angle and was reflected as such in the data. The regions of increased drag, around wind azimuth angles of $0^{\circ}$ and $180^{\circ}$, may have been caused by local flow acceleration (Venturi effect) around and between the nearby structures.

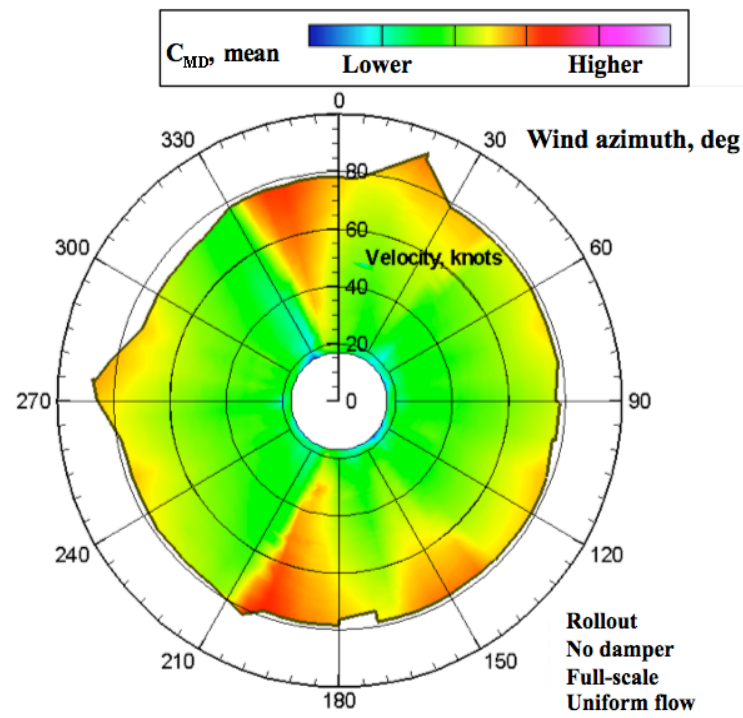

Figure 91. Ares I-X GWL Model. Normalized mean bending moment coefficient due to drag, rollout configuration. Full-scale values. 


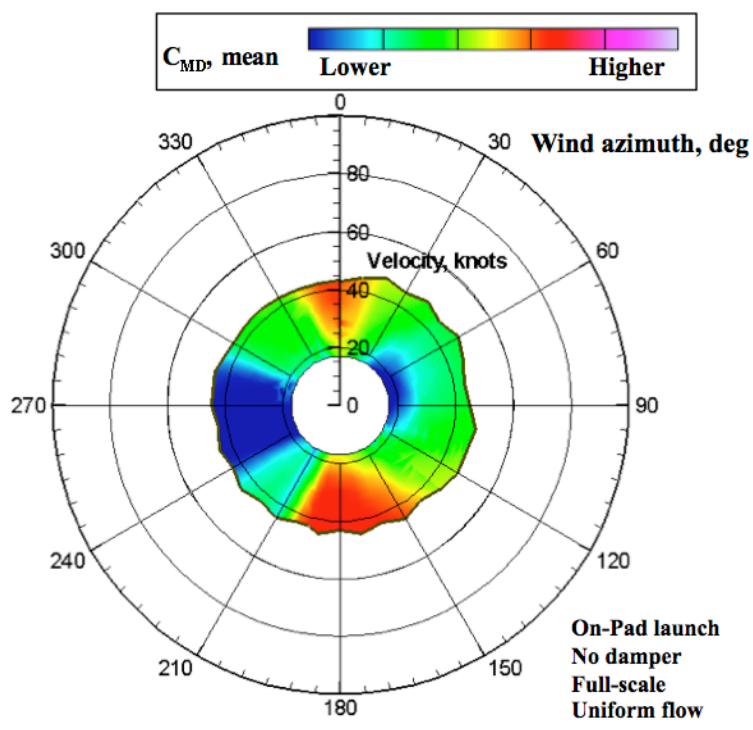

Figure 92. Ares I-X GWL Model. Normalized mean bending moment coefficient due to drag, on-pad launch configuration. Full-scale values.

\section{Ares I-X FTV (full-scale)}

The Ares I-X GWL Model effort was somewhat unique in that the full-scale Ares I-X FTV and MLP was instrumented to acquire vehicle base bending moment and response data, primarily for vehicle safety, along with wind data during rollout from the VAB to the KSC LC-39B and during selected periods while the Ares I-X FTV was at LC-39B (on-pad stay and launch). In addition, a modal test and analysis was performed on the Ares I-X FTV on the MLP in the VAB a few weeks prior to rollout. This presented an opportunity to acquire full-scale vehicle modal and GWL data for comparison to the wind tunnel model modal and wind-tunnel test results. Unfortunately, except for the modal test results and some limited vehicle response observed during low and moderate winds while on the pad, the data acquired during rollout and on-pad stay was of insufficient accuracy and reliability to conduct any type of rigorous correlation to the wind tunnel test results.

1. Modal Test and Analysis Prior to Rollout

In August of 2009 a modal test and analysis was performed on the full-scale Ares I-X FTV in the KSC Vehicle Assembly Building in preparation for its rollout and launch in October 2009. The Ares I-X FTV was mounted on the MLP for the modal test as it would be during rollout to and stay at the KSC LC-39B (Pad 39B). The modal test is reported in Ref. 12. The primary objective of the modal test was to obtain frequencies, mode shapes, and damping values for all critical vehicle modes of vibration to verify the dynamic FEM used in loads assessment and flight control evaluations. This modal test also allowed a comparison of the frequencies, mode shapes, and damping values between the full-scale vehicle and the Ares I-X FTV model as tested in the TDT. A comparison between the full-scale vehicle and the wind-tunnel model, rollout configuration, is presented in Table 17. It is the authors' opinion that full-scale vehicle values for damping of modes $2 \mathrm{Y}$ and $2 \mathrm{Z}$ were obtained at levels significantly lower than would occur at even modest levels of wind-induced motion, and therefore of questionable value for comparison, but are included here for completeness. In general, the Ares I-X FTV model was seen as a sufficiently accurate simulation of the full-scale dynamic characteristics for the four critical modes of concern for GWL and WIO testing.

Table 17. Comparison of Ares I-X FTV model and full-scale modal test results, frequencies and estimated damping. Rollout configuration. Model frequency results in full-scale.

\begin{tabular}{|c|c|c|c|c|}
\hline Mode & Full-scale, $\mathbf{H z}$ & Model, Hz. & Full-scale Damping, \%Cr & Model Damping, \%Cr \\
\hline $1(1 \mathrm{Y})$ & 0.18 & 0.17 & 0.85 & 0.46 \\
\hline $2(1 \mathrm{Z})$ & 0.21 & 0.21 & 0.43 & 0.38 \\
\hline $3(2 \mathrm{Y})$ & 1.06 & 0.92 & $0.29^{*}$ & 0.40 \\
\hline $4(2 \mathrm{Z})$ & 1.19 & 1.10 & $0.37^{*}$ & 0.74 \\
\hline
\end{tabular}


*Insufficient excitation for accurate determination of representative damping values.

\section{Ares I-X FTV and MLP Instrumentation and KSC LC-39B Wind Sensors}

Data available during the October 20, 2009 rollout of the Ares I-X FTV that was most relevant to ground wind loads were triaxial accelerometers located on the Ares I-X FTV at stations (inches) 785 and 1966, hold down post (HDP) strains (that were used to determine base bending moments), and a wind sensor mounted on the northeast corner (when located at LC-39B) of the MLP. In addition, there were two wind sensors mounted 60 feet above ground level (AGL), one located southeast and the other located northwest of LC-39B, approximately one quarter of a mile from the Ares I-X FTV when it was at LC-39B. All three wind sensors provided wind speed and azimuth data. The location and types of instrumentation on the Ares I-X FTV and MLP are depicted in Fig. 93 and the nearby LC-39B wind towers and sensors illustrated in Fig. 94. It should be noted that the MLP wind sensor and vehicle triaxial accelerometers were only functional, as planned, during rollout and immediately after rollout was completed. The wind sensor Se of the launch pad was not functioning properly during rollout and no reliable wind data from that sensor was available on the day of rollout. In addition, the remaining primary GWL data sensors, HDP strains and nearby wind sensors, were active and data acquired only in the few hours prior to the launch attempt that was scrubbed on October 27, 2009 and the Ares I-X FTV launch that occurred on October 28, 2009.

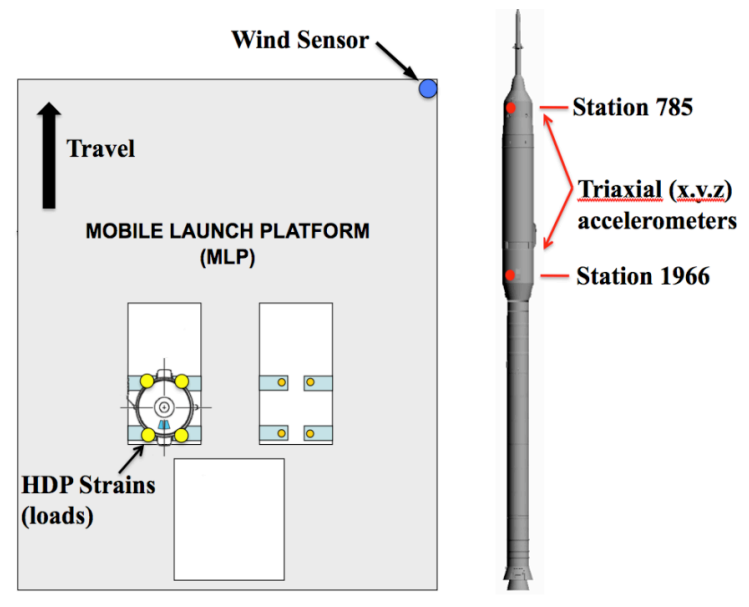

Figure 93. GWL related instrumentation on Ares I-X FTV and MLP.

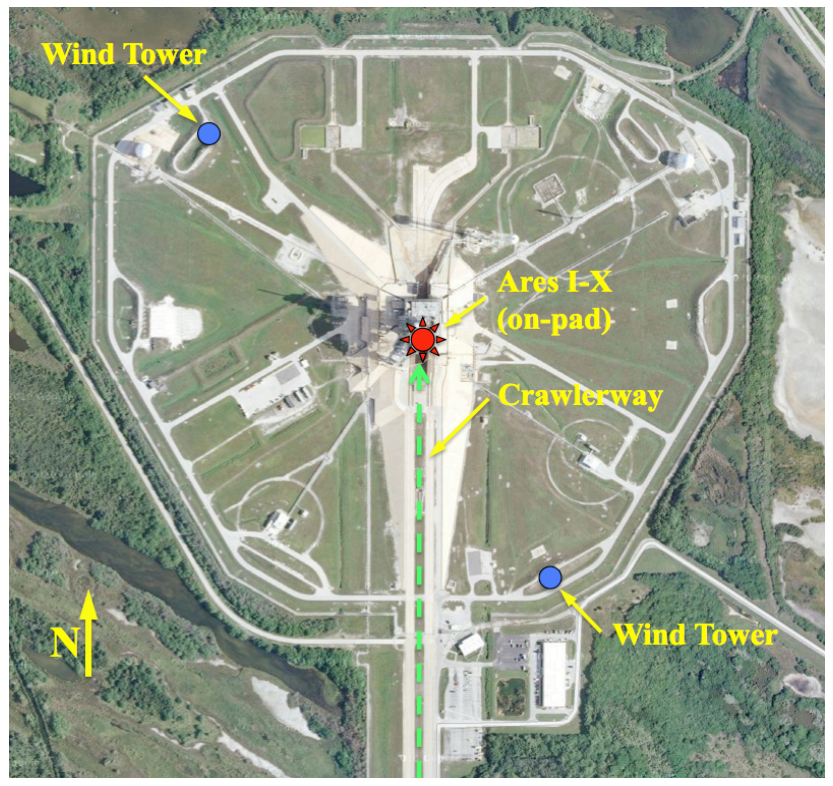

Figure 94. Wind tower locations at KSC LC-39B for Ares I-X FTV rollout and on-pad stay. 
In addition to these instrumentation issues and limitations, there were other aspects of the wind and HDP data that limited comparison of wind-tunnel data to that acquired during rollout and on-pad stay of the Ares I-X FTV. During rollout, the MLP wind sensor indicated wind speeds significantly (as much as approximately $40 \%$ ) lower than the wind tower located NW of the launch pad although wind direction (generally out of the NE) was generally within good agreement. Wind speeds during rollout were generally low at 5 to 10 knots at the beginning of rollout increasing to 15 to 20 knots as the Ares I-X FTV reached and was secured at the launch pad. Assuming these wind sensors were properly calibrated and operational, it seems unlikely that free stream wind velocities were that different at the two locations and the difference in readings were likely caused by the proximity of the MLP and launch vehicle, and later the FSS and RSS, on the MLP mounted wind sensor. In addition, with wind speed and direction data from only one elevation at all locations, it was not possible to reliably estimate the wind profile impacting the length of the vehicle. As stated above, no additional wind data was acquired from the MLP wind sensor after October 20, 2009. It was known from the wind-tunnel test and inspection that the wind field around the Ares I-X FTV during on-pad stay would be significantly impacted by the proximity of the FSS and RSS so that the wind data from the two nearby wind towers was of very limited use in trying to calculate accurate bending moment and force coefficients. Ares I-X FTV response data provided by the vehicle mounted accelerometers indicated low response of the primary bending modes of the vehicle although there were also significant response of other modes of vibration related to the crawler transport motion over the crawlerway. After the Ares I-X FTV and MLP were secured to the pad and prior to attachment of the VSS, there was some sinusoidal vehicle response, primarily first bending modes, almost certainly caused by wind blowing across the vehicle. No significant WIO motion was observed, however, and it seems likely the motion was caused by some combination of turbulence and gust response in addition to vortex shedding. Similar low amplitude response was observed in the HDP data during rollout and on-pad stay for all three days during data was acquired. HDP gauge drift seemed to occur between October 20, 2009 and October 27, 2009 that made calculation of mean base bending moments unreliable. Finally, once the Vehicle Stabilization System (VSS) was attached after completion of rollout and once the launch was scrubbed on October 27, 2009, all vehicle motion ceased and no load or response data could be acquired for correlation to windtunnel results. Wind speed and direction measured at the two nearby wind towers were generally 10 to 20 knots out of the SSE and 5 to 12 knots out of the SSW to SW for October 27, 2009 and October 28, 2009, respectively. Overall, while some interesting vehicle response and HDP data was acquired, it was not of sufficient quality for comparison to wind-tunnel data and no full-scale GWL data is presented in this paper.

\section{Summary}

The wind-tunnel investigation of the effects of ground winds on the Ares 1 launch vehicles indicated the possibility of not only classical first bending mode but also second bending mode WIO could occur and be a potential operational issue during rollout if no damper was present or the vehicle had low inherent structural damping, as was the case for the wind tunnel test models. The observation of second mode WIO during testing of the Ares I-X model, though a primary objective of the Ares I-X GWL Model test, appears to be a unique finding for a launch vehicle. The Ares I vehicle is unusual for its long slender shape and relatively low frequency second bending modes. The experimental data also indicated the importance of protuberances in determining critical wind conditions where WIO could occur on the full-scale vehicle. Overall, the wind-tunnel test program was successful in obtaining high quality data, within known limitations for this type of testing, and restored the capability and expertise required to do this type of testing at NASA. The extensive database obtained during the two wind tunnel tests should be useful in understanding WIO on this and similar launch vehicles and in the continued development and verification of computational methods for predicting GWL and specifically WIO. Currently, computational methods are not able to adequately predict WIO and dynamic response to ground winds due to the challenging nature of highly separated flows, vehicle protuberances, and effects of nearby structures. GWL wind-tunnel tests have known limitations in replicating vehicle and wind characteristics and extensive full-scale data from the Ares I$\mathrm{X}$ rollout and on-pad stay would have been valuable in evaluating the wind-tunnel data. However, the limited nature and duration of the full-scale data, the acknowledgement that critical wind conditions observed in the wind-tunnel tests were not present in the acquired full-scale data, and no significant WIO observed, resulted in a very limited comparison of the test data to full-scale results. More extensive full-scale data would have been be required for a proper evaluation of the wind-tunnel data.

\section{Acknowledgments}

Although the NASA Langley Research Center Aeroelasticity Branch led, participated, and performed a significant portion of the work involved in this model and wind-tunnel test program, there were other organizations 
and one contractor, in particular, that deserve recognition for their hard work and contributions to its success. The Aeronautics Systems Engineering Branch at NASA LaRC performed the detailed design for the entire GWL CM and the design of the FSS, RSS, and MLP simulator for the Ares I-X GWL model. These detailed designs were then fabricated and delivered by the LaRC Advanced Machining Development Section, the staff of which worked many overtime hours to allow the effort to meet a challenging schedule. The authors also wish to acknowledge the LaRC Aerospace Composite Model Development Section whose work included final fabrication of the Ares I-X GWL Model MLP simulator and pressure instrumentation integration on the GWL CM. The detailed design and fabrication of the Ares I-X FTV model was conducted by Micro Craft Inc. of Tullahoma, TN (now a unit of ATK Missile Products). Scott Ralston, model designer and engineer at Micro Craft, deserves special recognition for the quality of his work and dedication to making sure the launch vehicle model met or exceeded all NASA requirements and for working closely with the authors of this paper in such a professional and quality manner and being responsible for the on-time delivery of the model to NASA LaRC. Finally, the authors wish to thank the staff of the NASA LaRC Transonic Dynamics Tunnel for their excellent support of the two wind-tunnel tests during which they were responsible for installation of the model and supporting hardware and operation of the TDT under the direction of the authors.

\section{References}

${ }^{1}$ Jones, G. W. and Farmer, M. G., "Wind Tunnel Studies of Ground-Wind Loads on Saturn Launch Vehicles." AIAA Journal of Spacecraft, Volume 4, No 2. February 1967, pp 219-223.

${ }^{2}$ Jones, G. W., "Unsteady Lift Forces Generated by Vortex Shedding about a Large Stationary and Oscillating Cylinder at High Reynolds Numbers.” ASME Symposium on Unsteady Flow, Philadelphia, PA, May 1968, ASME 68-FE-36.

${ }^{3}$ Prelaunch Ground Wind Loads. NASA Space Vehicle Design Criteria, NASA SP 8008, NASA, November 1965.

${ }^{4}$ Chen, Shoei-Sheng., Flow-Induced Vibration of Circular Cylindrical Structures., Hemisphere Publishing Corporation, Washington, D.C., 1987.

${ }^{5}$ Terrestrial Environment (Climatic) Criteria Handbook for Use in Aerospace Vehicle Development, NASA-HDBK-1001, August 2000.

${ }^{6}$ Hanson, P. W. and Jones, G. W., "On the Use of Dynamic Models for Studying Launch Vehicle Buffet and Ground-Wind Loads.”, NASA TM X 50548, September 1963.

${ }^{7}$ Response of Structures to Vortex Shedding. Structures of Circular or Polygonal Cross Section. Engineering Sciences Data Unit (ESDU), Report 96030, December 1996. Amended July 1998.

${ }^{8}$ Szechenyi, E., "Supercritical Reynolds Number Simulation for Two-Dimensional Flow Over Circular Cylinders.", Journal of Fluid Mechanics, Volume 70, part 3, 1975, pp. 529-542.

${ }^{9}$ McCullough, G. B., and Steinmetz, W. J., "A Wind-Tunnel Study of Ground-Wind Loads on Launch Vehicles Including the Effects of Conduits and Adjacent Structures", NASA TN D-2889, July 1965.

${ }^{10}$ Roshko, A, "Experiments On the Flow Past a Circular Cylinder at Very High Reynolds Number", Journal of Fluid Mechanics, Volume 10, No. 3, 1961, pp. 345 - 356.

\footnotetext{
${ }^{11}$ Jones, G. W., Cincotta, J. J., and Walker, R. W., "Aerodynamic Forces on a Stationary and Oscillating Cylinder at High Reynolds Number”, NASA TR R-300, February 1969.

${ }^{12}$ Buehrle, R. D., Templeton, J. D., Reaves, M. C., Horta, L. G., and Gaspar, J. L., “Ares I-X Flight Test Vehicle Modal Test", NASA TM 2010-216182, January 2010.
} 